\title{
Shuffle algebras and perverse sheaves
}

\author{
Mikhail Kapranov, Vadim Schechtman
}

January 14,2020

to Kyoji Saito for his 75 th birthday

\begin{abstract}
We relate shuffle algebras, as defined by Nichols, Feigin-Odesskii and Rosso, to perverse sheaves on symmetric products of the complex line (i.e., on the spaces of monic polynomials stratified by multiplicities of roots). More precisely, we construct an equivalence between:

(i) Braided Hopf algebras of a certain type.

(ii) Factorizable collections of perverse sheaves on all the symmetric products.

Under this eqiuvalence, the Nichols algebra associated to an object $V$ corresponds to the collection of the intersection cohomology extensions of the local systems on the open configuration spaces associated to the tensor powers of $V$. Our approach is based on using real skeleta of complex configuration spaces.
\end{abstract}

\section{Contents}

1 Perverse sheaves and "bisheaves" on skeleta $\quad 7$

1.1 Skeleta pure with respect to a complex stratification . . . . . . . . . . . 7

1.2 Sheaves and bisheaves . . . . . . . . . . . . . . . . . . 11

1.3 Pure skeleta as degenerations of real fronts. Takeuchi's theorem . . . . . . . 12

1.4 Perverse sheaves with values in an abelian category . . . . . . . . . . . 14

2 Bisheaves and bialgebras: perverse sheaves on symmetric products $\quad 16$

2.1 The symmetric product $\operatorname{Sym}^{n}(\mathbb{C})$, its complex strata and real skeleton . . . 16

2.2 Imaginary strata and fine cells . . . . . . . . . . . . . . . . . . . . . 19

2.3 Perverse sheaves on $\operatorname{Sym}^{n}(\mathbb{C})$ : generalities . . . . . . . . . . . . . . . 21

2.4 Expected answer: bar-construction for bialgebras . . . . . . . . . . . 24

3 Factorizable perverse sheaves and bialgebras $\quad 29$

3.1 Shuffle algebras in braided categories . . . . . . . . . . . . . . . . . . . 29

3.2 Factorizable perverse sheaves . . . . . . . . . . . . . . . . . . 31

3.3 From bialgebras to factorizable sheaves: the localization theorem . . . . . 34 
4 Proof of Localization Theorem: Cousin complexes 38

4.1 The Cousin sheaves of a coalgebra . . . . . . . . . . . . . . . 38

4.2 The Cousin complexes of a bialgebra as perverse sheaves . . . . . . . . . 42

4.3 Factorizability of Cousin complexes: proof of Theorem $3.3 .1 \ldots \ldots$. . . . . 52

4.4 Perverse sheaves corresponding to shuffle algebras: proof of Theorem 3.3.3 . 55

5 Examples: Granger-Maisonobe quivers and bialgebras (Cases $n=2,3$ ) $\quad \mathbf{5 7}$

5.1 Generalities: reducing translation invariance . . . . . . . . . . 57

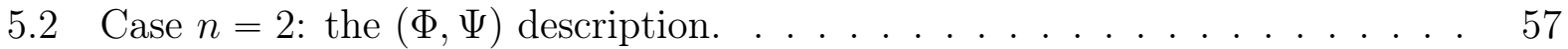

5.3 Case $\mathrm{n}=3$ : the Granger-Maisonobe quiver. . . . . . . . . . . . . 58

$\begin{array}{ll}\text { A Shuffle complexes } & 64\end{array}$

$\begin{array}{ll}\text { References } & 69\end{array}$

\section{Introduction}

A. First goal of the paper. Shuffle algebras were introduced by Feigin-Odesskii [17] and Rosso [52] but a closely related construction goes back to the 1978 paper of Nichols [49]. In modern terms, an object $V$ of a braided monoidal abelian category $(\mathcal{V}, \otimes, R)$ gives rise to three bialgebras in $\mathcal{V}$ :

(1) The tensor algebra $T_{!}(V)=\oplus_{n \geqslant 0} V^{\otimes n}$ with tensor multiplication and with the comultipliction making $V$ primitive.

(2) The cotensor, or "big shuffle" algebra $T_{*}(V)$ which is the same object $\bigoplus_{n \geqslant 0} V^{\otimes n}$ but with the shuffle multiplication involving the $R$-matrices, see (3.1.1) below. We have a canonical morphism $\alpha: T_{!}(V) \rightarrow T_{*}(V)$.

(3) The Nichols algebra (or the "true shuffle algebra") $T_{!_{*}}(V)$ defined as the image of $\alpha$.

For instance, the positive part $U_{q}^{+}(\mathfrak{g})$ of the quantum group with Cartan matrix $\left\|a_{i j}\right\|_{i, j=1}^{r}$ is obtained as $T_{\text {!* }}(V)$ for $V$ an object of the category of $\mathbb{Z}^{r}$-graded vector spaces with braiding given by the factors $q^{a_{i j}}$, see [17], [52] and Example 3.1.5 below for a precise formulation.

The first goal of this paper is to relate the above structures with the familiar phenomenon in the theory of perverse sheaves. If $X$ is a complex algebraic variety and $j: U \hookrightarrow X$ is an affine open subset, then a local system $\mathcal{L}$ on $U$ gives three perverse sheaves on $X$ :

$\left(1^{\prime}\right)$ The zero extension $j_{!} \mathcal{L}$.

$\left(2^{\prime}\right)$ The full direct image $j_{*} \mathcal{L}$. We have a canonical morphism $\alpha^{\prime}: j_{!} \mathcal{L} \rightarrow j_{*} \mathcal{L}$.

$\left(3^{\prime}\right)$ The intermediate (or intersection cohomology) extension $j_{! *} \mathcal{L}$ which is the image of $\alpha^{\prime}$. 
It turns out that (1)-(3) can be interpreted as a particular case of $\left(1^{\prime}\right)-\left(3^{\prime}\right)$, if we take for $X$ the symmetric products $\operatorname{Sym}^{n}(\mathbb{C})$, put $U=\operatorname{Sym}_{\neq}^{n}(\mathbb{C})$ to be the generic stratum and take $\mathcal{L}$ to be the local system on $U$ corresponding to the object $V^{\otimes n}$ with its natural action of the braid group. Here we consider all $n$ at once and work with local systems and perverse sheaves with values in $\mathcal{V}$.

Our interpretation can be seen as a nonlinear analog of several "localization" results, starting from the Beilinson-Bernstein picture [6], which deal with modules over a given (Lie) algebra, not algebras themselves. In such results, $\left(1^{\prime}\right)$ and $\left(2^{\prime}\right)$ correspond to the induced and co-induced Verma modules, $\alpha^{\prime}$ to the Shapovalov form and $\left(3^{\prime}\right)$ to the irreducible quotient of the Verma.

B. Second goal: skeletology. Our second goal is to develop, a little further, the method of totally real skeleta in the study of perverse sheaves i.e., in describing the category $\operatorname{Perv}(X, S)$ for a stratified complex manifold $(X, S)$. While the full range of this method is still not clear to us, in good cases [22] [32] [34] it works like this. We choose an appropriate "skeleton" (totally real subanalytic subset) $i: K \hookrightarrow X$ with the properties:

- For any $\mathcal{F} \in \operatorname{Perv}(X, S)$, the complex $i^{!} \mathcal{F}$ is quasi-isomorphic to a single sheaf $R_{K}(\mathcal{F})$. Therefore, taking the stalk of $R_{K}(\mathcal{F})$ at any given $x \in K$ gives an exact functor $R_{K, x}: \operatorname{Perv}(X, S) \rightarrow$ Vect.

- The functors $R_{K, x}$ take Verdier duality (denoted $\mathcal{F} \mapsto \mathbb{D} \mathcal{F}$ ) to vector space duality. Therefore the sheaf structure on $R_{K}(\mathbb{D} \mathcal{F})$ defines an extra cosheaf structure on $R_{K}(\mathcal{F})$ with the same (co)stalks.

- The sheaf and cosheaf structures on $R_{K}(\mathcal{F})$ are compatible in a certain sense. Spelling out these compatibility conditions amounts to defining the notion of a bisheaf on $K$. The category $\operatorname{Perv}(X, S)$ is then identified with the category of such bisheaves.

In the above situation of $X=\operatorname{Sym}^{n}(\mathbb{C})$ with its natural "diagonal" stratification, we can take $K=\operatorname{Sym}^{n}(\mathbb{R})$. In this case a source of "bisheaves" can be found in graded bialgebras: such a bialgebra gives a bisheaf on $K$ encoding the degree $n$ parts of the two Hochschild complexes: for the multiplication and comultiplication.

C. Description of results. As far as the first goal goes, we establish, in Theorem 3.3.1, an equivalence of categories $L$ between:

(1) A certain class of graded Hopf algebras (called primitive bialgebras, see Definition 2.4.10) in an abelian braided category $\mathcal{V}$.

(2) Perverse sheaves on $\operatorname{Sym}(\mathbb{C})=\bigsqcup_{n \geqslant 0} \operatorname{Sym}^{n}(\mathbb{C})$ (i.e., collections of perverse sheaves $\mathcal{F}_{n}$ on $\operatorname{Sym}^{n}(\mathbb{C})$ for all $n \geqslant 0$ ) with values in $\mathcal{V}$ which are:

(2a) Smooth with respect to the diagonal stratification, and 
(2b) Factorizable, that is, are equipped with a natural multiplicative structure with respect to the natural semigroup structure on $\operatorname{Sym}(\mathbb{C})$, see Definition 3.2.5.

We further prove, in Theorem 3.3.3, that the functor $L$ takes the tensor, cotensor and Nichols algebras to the perverse sheaves obtained as $j_{!}, j_{*}$ and $j_{! *}$ as explained above in $\S \mathrm{A}$.

As far as the second goal goes, we use the skeletal philosophy to connect, directly, bialgebras with "bisheaves" (and to motivate the idea of bisheaves in the first place). We also use it to give a very simple proof of the result of Takeuchi (quoted in Proposition 1.3.3) on the dimension of the linear algebra data (cohomology with support in $\mathbb{R}^{n}$ ) of a perverse sheaf on $\mathbb{C}^{n}$ smooth with respect to the complexification of a real hyperplane arrangement.

D. Summary of the paper. In Ch. 1 we discuss, at some length, our philosophy of skeleta and bisheaves ( $\S \S 1.1$ and 1.2 ). In $\S 1.3$, we consider examples of representation of skeleta as limit positions of deformations of "real fronts" $\{\operatorname{Re}(f) \geqslant 0\}$ of holomorphic functions $f$ and give a simple proof of Takeuchi's theorem (Prop. 1.3.3). In $\S 1.4$ we give a (rather routine) formal treatment of perverse sheaves with values in an abelian category, the concept which will be used later in the paper. Here it is convenient to use the sub-analytic topology of Kashiwara-Schapira [31].

In Ch. 2 we introduce our main object of study: the symmetric product $\operatorname{Sym}^{n}(\mathbb{C})$ together with its natural diagonal stratification. In particular, we "break the symmetry" of purely complex geometry, by introducing, in $§ 2.2$, the imaginary stratification and the decomposition into fine cells (extension of the Fox-Neuwirth cells decomposition of $\operatorname{Sym}_{\neq}^{n}(\mathbb{C})$ ). This allows us to construct, for any perverse sheaf $\mathcal{F}$ smooth with respect to the diagonal stratification, a natural Cousin resolution, an explicir complex of sheaves $\mathcal{E}^{\bullet}(\mathcal{F})$ quasi-isomorphic to $\mathcal{F}$, see Proposition 2.3.7. In $\S 2.4$ we introduce a natural class of examples of "bisheaves" on the real skeleton: the data encoding both the bar and cobar complex of a braided bialgebra. In particular, we introduce, in Definition 2.4.10 a concept of primitive bialgebras. Such bialgebras will be later (Theorem 3.3.1) identified with factorizable perverse sheaves.

In Ch. 3 we, first of all, recall the formalism of shuffle algebras in the generality we need (§3.1). In $\S 3.2$ we introduce the concept of factorizable perverse sheaves with values in a braided category. For this it is convenient to use the definition of a braided tensor structure based on the $E_{2}$-operad. In $\S 3.3$ we formulate our main results: the Localization Theorem 3.3.1 on equivalence between primitve bialgebras and factorizable perverse sheaves and Theorem 3.3.3 identifying perverse sheaves corresponding to shuffle and Nichols algebras.

Ch. 4 is devoted to the proof of Theorems 3.3.1 and 3.3.3 by a very direct method. Starting from a primitive bialgebra $A$, we produce, for each $n$, an explicit complex of sheaves $\mathcal{E}_{n}^{\bullet}(A)$ on $\operatorname{Sym}^{n}(\mathbb{C})$. A priori, it is only $\mathbb{R}$-constructible with respect to the stratification into fine cells, but we prove that it is in fact, a factorizable perverse sheaf with respect to the complex (diagonal) stratification. This remarkable phenomenon (the stalks of the complex $\mathcal{E}_{n}^{\bullet}(A)$ jump at real "walls" while their cohomology does not) comes from contractibility of some 
combinatorial complexes which appear as multiplicities and which are treated systematically in Appendix A.

In Ch. 5, which does not depend on the rest of the paper, we illustrate Theorem 3.3.1 using well known descriptions of perverse sheaves on $\operatorname{Sym}^{n}(\mathbb{C})$ for small values of $n$ : the classical $(\Phi, \Psi)$-description for $n=2$ and the Granger-Maisonobe description [26] for $n=3$.

Finally, Appendix A discusses various incarnations of a classical combinatorial object, the shuffle complex, whose vertices are shuffles of some type $\left(d_{1}, \cdots, d_{m}\right)$, edges correspond to elementary moves etc. Complexes of this nature appear as multiplicities in the stalks of the Cousin complexes in $\S 4$.

E. Relation to previous work. In [10] (inspired in turn by [53]), intermediate extensions of local systems on configuration spaces have been used to construct the Hochschild complex for the positive part of a small quantum group with coefficients in a tensor product of irreducible modules. In particular, one gets in this way the weight components of the irreducible modules themselves.

To achieve this goal one works at first on Cartesian configuration spaces (i.e., open parts of Cartesian, not symmetric products of $\mathbb{C}$ ) and then passes to invariants with respect to actions of the appropriate symmetric groups.

Unlike [10] (and earlier precursors), we work directly on the symmetric products $\operatorname{Sym}^{n}(\mathbb{C})$ rather than Cartesian powers $\mathbb{C}^{n}$ with diagonal stratifications. The advantage of this approach is that the linear algebra data have much smaller size (for $n=2$ we get the classical $(\Phi, \Psi)$-description) and match the features of a graded Hopf algebra very directly.

We have been very much influenced by the unpublished preprint of D. Gaitsgory [20] on factorizable algebras on configuration spaces. The method of [20] (see also [21], Theorem 29.2.3) is based on the Koszul duality relation between bialgebras and $E_{2}$-algebras, a purely "derived" concept which goes back to the works of Tamarkin and Kadeishvili, see [57], [58], [28], [12], and on the correspondence between $E_{2}$-algebras and factorizable constructible complexes on Ran spaces due to Lurie [41].

Unlike [20], our approach does not use $E_{2}$-algebras (even though the $E_{2}$-operad provides a natural language for defining braided categories). Instead, we produce the factorizable perverse sheaf $L(A)$ associated to a bialgebra $A$ in a completely direct and explicit way: as the sequence of Cousin complexes, using the $2 n$-dimensional real geometry of the "imaginary strata" in $\operatorname{Sym}^{n}(\mathbb{C})$. In fact, this geometry may be useful for constructing manageable models for the dg-operad $\mathbf{e}_{2}$ (singular chains of the topological operad $E_{2}$ ).

More recently, Ellenberg, Tran and Westerland [15], working in a general braided context, identified the Ext-cohomology of $T_{*}(V)$ with the homology of the braid group $\mathrm{Br}_{n}$ with coefficients in $V^{\otimes n}$ (twisted by the sign character). This result (Corollary 4.5 of [15]) is recovered, in an equivalent form, as our Corollary 3.3.4. An example of particular interest for [15] is $\mathcal{V}$ being the category of Yetter-Drinfeld modules for a finite group $G$ and $V$ being associated to a conjugation invariant subset $C \subset G$. In this case $V^{\otimes n}$ is the permutation 
representation associated to the natural action of $\mathrm{Br}_{n}$ on $C^{n}$, and its homology is identified with the topological homology of the Hurwitz space $\operatorname{Hur}_{G, n}^{C}$, the unramified cover of $\operatorname{Sym}_{\neq}^{n}(\mathbb{C})$ associated to the $\mathrm{Br}_{n}$-set $C^{n}$, see $[16,15]$ for background. Our approach puts these results into perspective and suggests a similar relation of the Nichols algebra $T_{! *}(V)$ (for $V$ associated to $G, C)$ to intersection homology of some compactifications of $\operatorname{Hur}_{G, n}^{C}$,

F. Future directions. Our approach, being completely elementary, suggests various generalizations, of which we mention two.

(1) In this paper we consider only non-linear objects: graded Hopf algebras $A$. One can similarly describe, in terms of perverse sheaves, the natural linear objects: Yetter-Drinfeld modules over $A$ (which form an abelian category) [61] [9]. For this, we need to use the same symmetric products $\operatorname{Sym}^{n}(\mathbb{C})$ but with different stratifications: choose a distinguished point $x_{0} \in \mathbb{C}$, say $x_{0}=0$, and stratify $\operatorname{Sym}^{n}(\mathbb{C})$ according to the multiplicity of $x_{0}$ (a separate nonnegative integer) and the collection of multiplicities at other points (an un-ordered partition). The natural real skeleton corresponding to this stratification is $\operatorname{Sym}^{n}\left(\mathbb{R}_{\geqslant 0}\right)$. After passing, as in [20], to $E_{2}$-algebras Koszul dual to bialgebras, this fits into the package of factorization algebras on $\mathbb{C}$ stratified by several points, see $[1,13,25]$. To further extend from $\mathbb{C}$ to more general Riemann surfaces, one needs to work in a ribbon braided category [2].

(2) The main context of the Feigin-Odesskii definition [17] of shuffle algebras (and of their many applications ever since) was that of $R$-matrices which depend meromorphically on a parameter $t \in C$ (with $C$ typically an algebraic curve). Conceptually, this corresponds to working in a meromorphic braided category [54], where one has some algebro-geometric structure on the "moduli space" of objects. It was pointed out by Kontsevich and Soibelman [38] that the parametric shuffle algebra gives rise to some factorization data on the cartesian products of $C$. The definition of these factorization data in [38] is similar to the definition from [10], but the data in [38] are of "coherent" nature (i.e., involve coherent sheaves). In the case of constant $R$-matrices, considered in the present paper, we deal with "constructible" data (perverse sheaves). It seems very plausible that these two directions: constructible and coherent, can be unified to give a conceptual interpretation of parametric shuffle algebras.

G. Acknowledgements. It is a pleasure to dedicate this work to Kyoji Saito. His proposal was at the origin of the Granger - Maisonobe example [26], which, in turn, was at the origin of our reflections on these topics.

We are grateful to D. Gaitsgory, V. Hinich, P. Safronov, K. Saito and C. Westerland for useful discussions. The research of M.K. was supported by World Premier International Research Center Initiative (WPI Initiative), MEXT, Japan and by the IAS School of Mathematics. V.S. is grateful to Kavli IPMU for the hospitality during the conference on vertex algebras in the summer 2018, where a part of the present work has been reported. 


\section{Perverse sheaves and "bisheaves" on skeleta}

\subsection{Skeleta pure with respect to a complex stratification}

A. Pure skeleta. We fix, once and for all, a base field $\mathbf{k}$ of characteristic 0 . We denote Vect $_{\mathbf{k}}$, resp. Vect $\mathrm{fd}_{\mathbf{k}}^{\mathrm{fd}}$ the category of all, resp. of finite-dimensional, $\mathbf{k}$-vector spaces.

Let $X$ be a connected complex manifold, $\operatorname{dim}_{\mathbb{C}}(X)=n$. Let $S=\left\{X_{\alpha}\right\}_{\alpha \in A}$ be a complex analytic Whitney stratification of $X$. We denote by $i_{\alpha}: X_{\alpha} \rightarrow X$ the embedding of the stratum $X_{\alpha}$. By $X_{0}$ we denote the unique open stratum of $S$, so $0 \in A$ is a distinguished element.

Let $\operatorname{Sh}(X, S)$ be the abelian category of sheaves of $\mathbf{k}$-vector spaces $\mathcal{F}$ on $X$ constructible with respect to $S$. This means $\mathcal{F}$ is locally constant on each $X_{\alpha}$, with finite-dimensional stalks. Let further $D^{b}(X, S)$ be the bounded derived category formed by complexes of sheaves $\mathcal{F}$ of $\mathbb{C}$-vector spaces on $X$ constructible with respect to $S$. This means each cohomology sheaf $\underline{H}^{i}(\mathcal{F})$ belongs to $\operatorname{Sh}(X, S)$.

Let $\operatorname{Perv}(X, S) \subset D^{b}(X, S)$ be the abelian category formed by perverse sheaves with respect to the middle perversity. Explicitly, see, e.g., [29] a complex $\mathcal{F}$ is perverse, if:

$\left(P^{+}\right)$For every $\alpha \in A$, we have $\underline{H}^{n}\left(i_{\alpha}^{*} \mathcal{F}\right)=0$ for $n>-\operatorname{dim}_{\mathbb{C}}\left(X_{\alpha}\right)$.

$\left(P^{-}\right)$For every $\alpha \in A$, we have $\underline{H}^{n}\left(i_{\alpha}^{!} \mathcal{F}\right)=0$ for $n<-\operatorname{dim}_{\mathbb{C}}\left(X_{\alpha}\right)$.

In particular, the conditions imply that the restriction of a perverse $\mathcal{F}$ to the open stratum $X_{0}$ is a local sysytem in degree $(-n)$. The category $\operatorname{Perv}(X, S)$ carries the Verdier duality $\mathcal{F} \mapsto \mathcal{F}^{*}$.

We will also denote

$$
D_{\text {constr }}^{b}(X)=\bigcup_{S} D^{b}(X, S), \quad \operatorname{Perv}(X)=\bigcup_{S} \operatorname{Perv}(X, S) \subset D_{\text {constr }}^{b}(X)
$$

the derived category of complexes of sheaves on $X$ constructible with respect to some (indeterminate) stratification and the subcategory of perverse sheaves there.

Let $K \subset X$ be a closed real subanalytic set of pure real dimension $n$. We denote $i_{K}: K \rightarrow X$ the embedding. The Whitney stratification $S$ induces a stratification (disjoint decomposition) $S_{K}$ of $K$ into strata $K_{\alpha}=K \cap X_{\alpha}$.

By a topological ball we will mean a subanalytic subset isomorphic, as such, to $\mathbb{R}^{m}$ for some $m$.

Definition 1.1.1. (a) $K$ is called a skeleton for $(X, S)$, if each $K_{\alpha}$ is a disjoint union of topological balls of the same dimension.

(b) A skeleton $K$ is called pure, if for any $\mathcal{F} \in \operatorname{Perv}(X, S)$ the complex $i_{K}^{!} \mathcal{F}$ on $K$ is quasi-isomorphic to a single sheaf in degree 0 , which we denote $R_{K}(\mathcal{F})=\underline{H}_{K}^{0}(\mathcal{F})$. 
We refer to the connected components of $K_{\alpha}$ as cells of $K$ and denote by $(\mathcal{C}, \leqslant)$ the set of cells of $K$, ordered by inclusion of closures.

Let $\operatorname{Sh}(K, \mathcal{C})$ be the abelian category of sheaves of $\mathbf{k}$-vector spaces on $K$ which are locally constant (hence constant) on each cell of $\mathcal{C}$, with finite-dimensional stalks.

If $K$ is a pure skeleton, we have an exact functor of abelian categories

$$
R_{K}=i_{K}^{!}: \operatorname{Perv}(X, S) \longrightarrow \operatorname{Sh}(K, \mathcal{C}) .
$$

In particular, for any cell $C \in \mathcal{C}$ we have an exact functor

$$
R_{K, C}: \operatorname{Perv}(X, S) \longrightarrow \operatorname{Vect}_{\mathbf{k}}^{\mathrm{fd}}, \quad \mathcal{F} \mapsto R_{K}(\mathcal{F})_{C}:=H^{0}\left(C, R_{K}(\mathcal{F})\right)
$$

obtained by taking the stalk of $R_{K}(\mathcal{F})$ at $C$ or, what is the same, at any point $x \in C$ (these stalks are canonically identified for different $x \in C)$.

B. Pure skeleta and quiver descriptions of $\operatorname{Perv}(X, S)$. In studying perverse sheaves, considerable effort had been spend on "elementary" descriprions of the categories $\operatorname{Perv}(X, S)$ for some particular stratified spaces $(X, S)$, see $[5,26,22,46]$. Typically, such a description has the form of an identification

$$
\operatorname{Perv}(X, S) \stackrel{\simeq}{\longrightarrow} \operatorname{Rep}(Q, R)
$$

with $\operatorname{Rep}(Q, R)$, the category of representations of some quiver (oriented graph) $Q$ subject to a set of relations $R$, a category which is manifestly abelian. An object of $\operatorname{Rep}(Q, R)$ is a family of vector spaces $V_{i} \in$ Vect (corresponding to the vertices of $Q$ ) and linear operators $a_{e}: V_{\text {source }(e)} \rightarrow V_{\text {target }(e)}$ for any oriented edge $e$ of $Q$, subject to the relations from the set $R$.

An identification (1.1.2) contains, in particular, a datum of several exact functors

$$
\operatorname{Perv}(X, S) \longrightarrow \operatorname{Vect}_{\mathbf{k}}^{\mathrm{fd}}, \quad \mathcal{F} \mapsto V_{i}(\mathcal{F})
$$

where the $V_{i}(\mathcal{F})$ are the vector spaces of the quiver representation corresponding to $\mathcal{F}$. Now, a priori it is not easy to construct such exact functors in the first place. But if $K$ is a pure skeleton for $(X, S)$, then we have a natural supply of such functors, namely the $R_{K, C}, C \in \mathcal{C}$.

Observation 1.1.3. In most examples, the $V_{i}(\mathcal{F})$ can indeed be found as $R_{K, C}(\mathcal{F})$ for an appropriate skeleton $K$. Further, the natural maps between the $R_{K, C}(\mathcal{F})$ coming from the structure of a sheaf on $R_{K}(\mathcal{F})$, provide precisely one-half of the arrows $a_{e}$ in the quiver description, the other half being the "formal adjoints" of these. The key to a quiver description consists therefore in finding a sufficiently rich pure skeleton for $(X, S)$.

Example 1.1.4 (Graphs on surfaces). Let $n=1$, i.e., $X$ be a Riemann surface. A stratification $S$ consists then of a discrete set $N \subset X$ so that the strata are $X \backslash N$ and the points of $N$. A skeleton for $(X, S)$ is nothing but a (subanalytic) graph $K$ embedded into 
$X$, regardless of its position with respect to $N$. In this case it is not hard to prove that every graph is in fact a pure skeleton, see [34], Prop. 2.2. By taking $K$ sufficiently rich (i.e., passing through all the points of $N$ and being a spanning graph for $X$ ), one can obtain from this approach a quiver description of $\operatorname{Perv}(X, N)$, see [34]. This contains the following more well known examples.

Example 1.1.5 (The $(\Phi, \Psi)$ description). Let $X=\mathbb{C}$ and let $N=\{0\}$ consist of one point. Denote the corresponding category of perverse sheaves Perv $(\mathbb{C}, 0)$. Take for $K$ the closed half-line $[0, \infty)$ through 0 . The cells of $K$ are $\{0\}$ and $(0, \infty)$. Given $\mathcal{F} \in \operatorname{Perv}(\mathbb{C}, 0)$, the stalks of the sheaf $R_{[0, \infty)}(\mathcal{F})=\underline{\mathbb{H}}_{[0, \infty)}^{0}(\mathcal{F})$ at these cells are $\Phi(\mathcal{F})$ and $\Psi(\mathcal{F})$, the classical spaces of vanishing and nearby cycles of $\mathcal{F}$.

Further, a constructible sheaf in $\operatorname{Sh}([0, \infty), \mathcal{C})$ is the same as a diagram $v: E_{0} \rightarrow E_{1}$, where $E_{0}$ is the stalk at $0, E_{1}$ the stalk at $(0, \infty)$ and $v$ is the generalization map. So the sheaf structure of $R_{[0, \infty)}(\mathcal{F})$ associates to $\mathcal{F}$ the arrow $\Phi(\mathcal{F}) \stackrel{v}{\rightarrow} \Psi(\mathcal{F})$ which is precisely one half of the two arrows in the classical description [22] [5] of $\operatorname{Perv}(\mathbb{C}, 0)$ as the category of diagrams

$$
\Phi \underset{u}{\stackrel{v}{\rightleftarrows}} \Psi, \quad T_{\Psi}:=\mathrm{Id}_{\Psi}-v u, T_{\Phi}=\mathrm{Id}_{\Phi}-u v \text { invertible. }
$$

We note that invertibility of $T_{\Psi}$ implies invertibility of $T_{\Phi}$ and vice versa (even without assuming finite dimensionality of $\Phi$ and $\Psi$ ), because of the identity

$$
(1-u v)^{-1}=1+u(1-v u)^{-1} v,
$$

familiar in the theory of the Jacobson radical, cf. [39] §3.2, proof of Proposition 4. We are grateful to V. Drinfeld for pointing it out to us.

Example 1.1.7 (The "Dirac" description). Keeping the same $X=\mathbb{C}$ and $S=\{\{0\}, \mathbb{C} \backslash\{0\}\}$ as above, we take $K=\mathbb{R}=(-\infty, \infty)$ the real line through 0 . This $K$ has three cells: $\mathbb{R}_{-}=(-\infty, 0),\{0\}$ and $\mathbb{R}_{+}=(0, \infty)$. A constructible sheaf in $\operatorname{Sh}(\mathbb{R}, \mathcal{C})$ is the same as a diagram of stalks at these cells and the generalization maps

$$
E_{-} \stackrel{\gamma_{-}}{\longleftarrow} E_{0} \stackrel{\gamma_{+}}{\longrightarrow} E_{+} .
$$

This corresponds to the so-caled "Dirac description" of $\operatorname{Perv}(\mathbb{C}, \mathcal{F})$, see [32] $\S 9 \mathrm{~A}$, in terms of the diagrams

$$
\begin{gathered}
E_{-} \underset{\delta_{-}}{\stackrel{\gamma_{-}}{\longrightarrow}} E_{0} \underset{\delta_{+}}{\stackrel{\gamma_{+}}{\longrightarrow}} E_{+}, \\
\gamma_{-} \delta_{-}=\operatorname{Id}_{E_{-}}, \gamma_{+} \delta_{+}=\operatorname{Id}_{E_{+}}, \quad \gamma_{-} \delta_{+}: E_{+} \rightarrow E_{-}, \quad \gamma_{+} \delta_{-}: E_{-} \rightarrow E_{+} \text {invertible. }
\end{gathered}
$$

Again, the structure of the sheaf $R_{\mathbb{R}}(\mathcal{F})$ for $\mathcal{F} \in \operatorname{Perv}(\mathbb{C}, 0)$ provides precisely one-half of the arrows in the diagram. 
Example 1.1.8 (Hyperplane arrangements). More generally, let $X=\mathbb{C}^{n}$ and let $\mathcal{H}=$ $\left\{H_{i}\right\}_{i \in I}$ be an arrangement of linear hyperplanes in $\mathbb{R}^{n}$. Then $\mathcal{H}_{\mathbb{C}}=\left\{H_{i}^{\mathbb{C}}=H_{i} \otimes \mathbb{C}\right)$ is an arrangement of hyperplanes in $\mathbb{C}^{n}$ and it gives a Whitney stratifications $S=S_{\mathcal{H}}$ into the strata

$$
L^{\circ}=L \backslash \bigcup_{H_{i} \ngtr L}\left(L \cap H_{i}^{\mathbb{C}}\right) .
$$

Here $L$ runs over flats of $\mathcal{H}_{\mathbb{C}}$, i.e., linear subspaces obtained as intersections $\bigcap_{j \in J} H_{j}^{\mathbb{C}}$ for any subset $J \subset I$ (including $J=\varnothing$ which corresponds to $L=\mathbb{C}^{n}$ ).

Denote the corresponding category of perverse sheaves $\operatorname{Perv}\left(\mathbb{C}^{n}, \mathcal{H}\right)$. It was studied in [32]. In particular, it was proved there that the real space $K=\mathbb{R}^{n} \subset \mathbb{C}^{n}=X$ is a pure skeleton for this stratification. The set $\mathcal{C}$ consists of faces (of all dimensions) of the real arrangement $\mathcal{H}$.

We recall, see, e.g., [32] §1D:

Definition 1.1.9. A regular cell decomposition of a space $X$ is a stratification into open topological balls (cells) such that the closure of any cell is a closed topological ball. A quasi-regular cell complex is a difference $X \backslash X^{\prime}$ of two regular cell complexes with $X^{\prime}$ a closed cellular subcomplex of $X$.

In the examples above (in fact, in most of the examples of skeleta we know), the stratification $S_{K}$ is a quasi-regular cell decomposition. For a quasi-regular cell complex, it is standard that taking the stalks at various cells and generalization maps among them gives an identification

$$
\operatorname{Sh}(K, \mathcal{C}) \simeq \operatorname{Rep}(\mathcal{C}):=\operatorname{Fun}\left((\mathcal{C}, \leqslant), \operatorname{Vect}_{\mathbf{k}}^{\mathrm{fd}}\right)
$$

Here $\operatorname{Rep}(\mathcal{C})$ is the category of representations of the poset $(\mathcal{C}, \leqslant)$, i.e., functors from $(\mathcal{C}, \leqslant)$ considered as a category, to Vect $\mathrm{fd}_{\mathbf{k}}^{\mathrm{fd}}$.

For more general cell decompositions the role of $(\mathcal{C}, \leqslant)$ is played by $\operatorname{Exit}(K, \mathcal{C})$, the category of exit paths of the stratification $\mathcal{C}$, see [59]. 


\subsection{Sheaves and bisheaves}

A. The idea of bisheaves. In the examples we have seen, the linear algebra data describing $\mathcal{F} \in \operatorname{Perv}(X, S)$ give, in particular, an actual constructible sheaf on the skeleton $K$, in terms of its stalks and generalization maps. But they also contain companion maps in the opposite direction which, similarly, give a cosheaf on $K$, that is, a sheaf with values in Vect ${ }_{\mathbf{k}}^{\text {op }}$, the opposite to the category of vector spaces.

It is suggestive, therefore, to introduce the term bisheaf to describe the entire set of linear algebra data representing $\mathcal{F}$. Informally, a bisheaf should consist of an $S_{K}$-constructible sheaf and cosheaf on $K$, with the same stalks at the cells, satisfying some compatibility conditions between the two structures.

Example 1.2.1. For instance, in the situation of Example 1.1 .7 we have $K=\mathbb{R}$ with stratification $K_{S}$ into $\mathbb{R}_{-},\{0\}$ and $\mathbb{R}_{+} \mathrm{A} S_{K^{-}}$-constructible sheaf on $K$ is the same as a diagram

$$
E_{-} \stackrel{\gamma_{-}}{\longleftarrow} E_{0} \stackrel{\gamma_{+}}{\longrightarrow} E_{+},
$$

a cosheaf is the same as a diagram

$$
E_{-} \stackrel{\delta_{-}}{\longrightarrow} E_{0} \stackrel{\delta_{+}}{\longleftarrow} E_{+},
$$

and a bisheaf should be a diagram

$$
E_{-} \underset{\delta_{-}}{\stackrel{\gamma_{-}}{\rightleftarrows}} E_{0} \underset{\delta_{+}}{\stackrel{\gamma_{+}}{\rightleftarrows}} E_{+},
$$

satisfying the conditions $\gamma_{ \pm} \delta_{ \pm}=\operatorname{Id}$ as well as the invertibility of $\gamma_{+} \delta_{-}$and $\gamma_{-} \delta_{+}$.

We do not know a general definition of a bisheaf. It seems that it should not be a purely topological concept involving the stratified space $\left(K, S_{K}\right)$ but should involve information about the complexification $(X, S)$ as well.

B. Self-dual skeleta. The skeleta that have been used, have, in addition to purity, the following property: the functors $R_{K, C}: \operatorname{Perv}(X, S) \rightarrow \operatorname{Vect}_{\mathbf{k}}^{\mathrm{fd}}$, take Verdier duality to the usual duality fo vector spaces, i.e., we have functorial isomorphisms

$$
R_{K}(\mathbb{D} \mathcal{F})_{C} \simeq\left(R_{K}(\mathcal{F})_{C}\right)^{*} .
$$

This duality then interchanges the sheaf and cosheaf structures. That is, the additional "cosheaf" structure on the sheaf $R_{K}(\mathcal{F})$ comes from the structure of sheaf on $R_{K}\left(\mathcal{F}^{*}\right)$.

We will call this property of a pure skeleton self-duality. Using the standard properties of Verdier duality (interchanging $i^{*}$ and $i^{!}$), for a self-dual skeleton $K$ we have natural isomorphisms

$$
i_{C}^{*} i_{K}^{!} \mathcal{F} \simeq i_{C}^{!} i_{K}^{*} \mathcal{F}, \quad \mathcal{F} \in \operatorname{Perv}(S, X), C \in \mathcal{C} .
$$




\subsection{Pure skeleta as degenerations of real fronts. Takeuchi's theorem}

Let $f$ be a holomorphic function on $X$. We then have the hypersurface $X_{f}=\{f=0\}$ and the closed subanalytic set $X_{f}^{+}=\{\operatorname{Re}(f) \geqslant 0\}$ which we call the real front of $f$. Denote

$$
X_{f} \stackrel{i_{f}}{\hookrightarrow} X_{f}^{+} \stackrel{j_{f}}{\hookrightarrow} X
$$

the embeddings. It is known [29] that the functor of vanishing cycles

$$
\Phi_{f}: D_{\text {constr }}^{b}(X) \longrightarrow D_{\text {constr }}^{b}\left(X_{f}\right)
$$

perserves perverse sheaves and commutes with Verdier duality. It is also known (ibid.) that we have a canonical quasi-isomorphism

$$
\Phi_{f}(\mathcal{F}) \simeq i_{f}^{*} j_{f}^{!}(\mathcal{F})
$$

On the other hand, for a skeleton $K$ and its cell $C$, the stalk at $C$ of the $\operatorname{complex} R_{K}(\mathcal{F})$ can be written as $i_{C}^{*} i_{K}^{!}(\mathcal{F})$. This suggests a way to construct pure and self-dual skeleta as limits of isotopic deformations of regions of the form $X_{f}^{+}$.

Example 1.3.1. (a) In the situation of Example 1.1.5, the skeleton $[0, \infty)$ can be seen as a limit of isotopic deformations (compatible with our stratification) of the real front $\operatorname{Re}(z) \geqslant 0$.
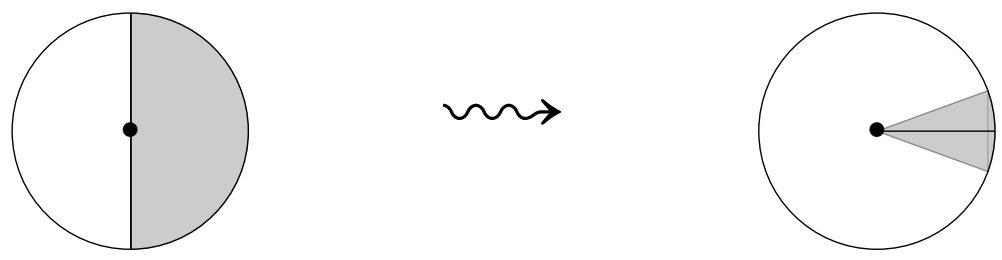

Figure 1: Deforming $\{\operatorname{Re}(z) \geqslant 0\}$ into the half-line.

Example 1.3.2. In the situation of Example 1.1.7, the skeleton $\mathbb{R}=(-\infty, \infty)$ can be seen as a limit of isotopic deformations of the real front $\left\{\operatorname{Re}\left(z^{2}\right) \geqslant 0\right\}$. The deformation is achieved by the transformations $T_{\varepsilon}: \mathbb{C} \rightarrow \mathbb{C}, 0 \leqslant \varepsilon \leqslant 1$, taking $x+i y$ to $x+\varepsilon i y$, see Fig. 2 . Note that such transformations cannot be made holomorphic, since we need to decrease the angle of aperture of the sectors. Nevetheless, they preserve the topology of the situation.
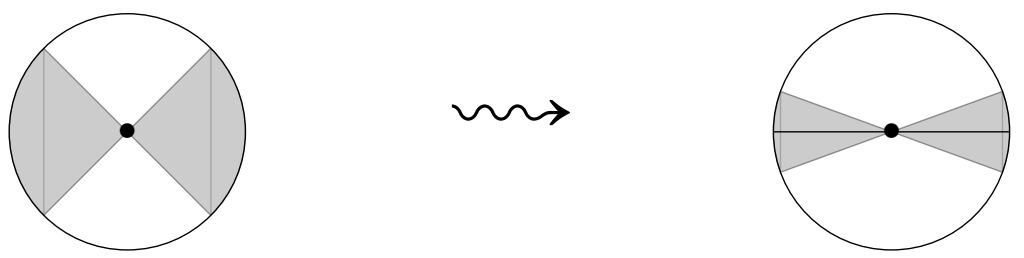

Figure 2: Deforming $\left\{\operatorname{Re}\left(z^{2}\right) \geqslant 0\right\}$ into the real line. 
Let us now consider the situation of Example 1.1.8, so we have an arrangement $\mathcal{H}$ of hyperplanes in $\mathbb{R}^{n}$ and the corresponding category $\operatorname{Perv}\left(\mathbb{C}^{n}, \mathcal{H}\right)$. In the following proposition both parts are known but rather non-trivial. Part (a), already mentioned in Example 1.1.8, was proved in [32] but an equivalent statement goes back to [10]. Part (b) is a result of Takeuchi [56].

Proposition 1.3.3. (a) The skeleton $\mathbb{R}^{n} \subset \mathbb{C}^{n}$ is pure and self-dual.

(b) For $\mathcal{F} \in \operatorname{Perv}\left(\mathbb{C}^{n}, \mathcal{H}\right)$ the dimension of the stalk $R_{\mathbb{R}^{n}}(\mathcal{F})_{0}$ is equal to

$$
\sum_{L} \operatorname{mult}_{T_{L}^{*} \mathbb{C}^{n}}(\mathcal{F})
$$

where $L$ runs over all complex flats of $\mathcal{H}$ and mult $_{T_{L}^{*} \mathbb{C}^{n}}(\mathcal{F})$ is the multiplicity of the Lagrangian variety $T_{L}^{*} \mathbb{C}^{n}$ in the characteristic cycle of $\mathcal{F}$.

We now present an extremely simple proof of both statements by the method of degenerations, developing a higher-dimensional version of Example 1.3.2. That is, we take a quadratic form $Q(z)$ on $\mathbb{C}^{n}$ with real coefficients such that the restriction of $Q$ to $\mathbb{R}^{n}$ is positive definite. Explicitly, we choose a linear coordinate system $x_{1}, \cdots, x_{n}$ on $\mathbb{R}^{n}$ and define

$$
Q: \mathbb{C}^{n} \rightarrow \mathbb{C}, \quad Q\left(z_{1}, \cdots, z_{n}\right)=\sum z_{i}^{2}
$$

The real part $\operatorname{Re}(Q): \mathbb{C}^{n} \rightarrow \mathbb{R}$ is a nondegenerate quadratic form of signature $(n, n)$, and the transformations

$$
T_{\varepsilon}: \mathbb{C}^{n} \rightarrow \mathbb{C}^{n}, \quad \mathbf{x}+i \mathbf{y} \mapsto \mathbf{x}+i \varepsilon \mathbf{y}, \quad 0 \leqslant \varepsilon \leqslant 1
$$

"flatten" the region $\operatorname{Re}(Q) \geqslant 0$ into $\mathbb{R}^{n}$ in a way compatible with the stratification $S_{\mathcal{H}}$, much like in Fig. 2. Therefore

$$
i_{0}^{*} i_{\mathbb{R}^{n}}(\mathcal{F}) \simeq \Phi_{Q}(\mathcal{F})_{0}
$$

is the stalk at 0 of the perverse sheaf of vanishing cycles $\Phi_{Q}(\mathcal{F})$. If we now assume that $\mathcal{H}$ is a central arrangement i.e., that $\{0\}$ is the minimal flat (we can always reduce to this case by quotienting by the minimal flat), then $\Phi_{Q}(\mathcal{F})$ is supported at 0 and so reduces to a single vector space in degree 0 . This shows that the stalk of $i_{\mathbb{R}^{n}}^{!}(\mathcal{F})$ at 0 , the minimal celll of the real arrangement, is concentrated in degree 0 . The stalk at any other cell $C$ is analyzed similarly, by taking the transversal slice to $C$ and using a positive definite quadratic form on that slice. This proves (a).

Further, (b) follows at once (1.3.4) and the standard microlocal interpretation of vanishing cycles [29]. That is, the cotangent space of $\mathbb{C}^{n}$ is $\mathbb{C}^{n} \times \mathbb{C}^{n *}$. The stratification $S=\left\{L^{\circ}\right\}$ gives rise to the set of Langangian varieties $T_{L}^{*} \mathbb{C}^{n}=L \times L^{\perp}$ which can contribute to the characteristic cycle of $\mathcal{F}$. The graph of $d Q$ is the Lagrangian variety $\Gamma_{d Q}$ which is the graph of the isomorphism $\mathbb{C}^{n} \rightarrow \mathbb{C}^{n *}$ given by the bilinear form corresponding to $Q$. It meets each 
$T_{L}^{*} \mathbb{C}^{n}$ exactly once, transversely, at the point $(0,0)$. Let us now deform $\Gamma_{d Q}$ a little, replacing $Q$ with $Q^{\prime}=Q+l$ where $l$ is a generic linear function on $\mathbb{C}^{n}$. Then

$$
\operatorname{dim} \Phi_{Q}(\mathcal{F})_{0}=\sum_{x \in \mathbb{C}^{n}} \operatorname{dim} \Phi_{Q^{\prime}}(\mathcal{F})_{x}
$$

But $\Gamma_{d} Q^{\prime}$ is a generic shift of $\Gamma^{\prime}$ which will meet each $T_{L}^{*} \mathbb{C}^{n}$ exactly once, transversely, at a point $\left(x_{L}, \xi_{L}\right)$ which does not belong to any other $T_{M}^{*} \mathbb{C}^{n}, M \neq L$. This implies that

$$
\operatorname{dim} \Phi_{Q^{\prime}}(\mathcal{F})_{x_{L}}=\operatorname{mult}_{T_{L}^{*} \mathbb{C}^{n}}(\mathcal{F})
$$

and the claim follows.

\subsection{Perverse sheaves with values in an abelian category}

In the remainder of the paper it will be convenient to work with sheaves and perverse sheaves formed not by vector spaces but by objects of some abelian category $\mathcal{V}$. In this section we formally sketch this concept. The reader can assume that $\mathcal{V}$ consists of vector spaces with some extra structure (e.g., of modules over a k-algebra $\Lambda$ ). In such a case the generalization we describe is really trivial (perverse sheaves with the same extra structure).

A. Sheaves with values in a Grothendieck category. Let $\mathcal{V}$ be an k-linear abelian category. Note that there is an intrinsic concept of tensoring an object $V$ of $\mathcal{V}$ with a finite-dimensional k-vector space $E$, the result of which will be denoted $V \otimes_{\mathbf{k}} E$.

Let us first assume that $\mathcal{V}$ is a Grothendieck category [30], in particular, that it has arbitrary direct sums and products. For example, one can take for $\mathcal{V}$ the category of all modules over a k-algebra $\Lambda$.

Let $X$ be a topological space. We can speak about sheaves on $X$ with values in $\mathcal{V}$. These are contravariant functors $\mathcal{F}$ from the poset of open sets in $X$ to $\mathcal{V}$ satisfying the descent axiom: for any open covering $U=\bigcup_{i \in I} U_{i}$ of an open set $U$, the sequence

$$
0 \rightarrow \mathcal{F}(U) \longrightarrow \prod_{i \in I} \mathcal{F}\left(U_{i}\right) \longrightarrow \prod_{i, j \in I} \mathcal{F}\left(U_{i} \cap U_{j}\right)
$$

is exact.

The operation of passing from a presheaf with values in $\mathcal{V}$ to the associated sheaf is defined as usual. A constant sheaf with values in $\mathcal{V}$ is the sheaf associated with a constant presheaf $(U \subset X) \mapsto$ a fixed $V \in \mathcal{V}$. A local system on $X$ with values in $\mathcal{V}$ is a sheaf locally isomorphic to a constant sheaf. It is the same as a functor from the fundamental groupoid $\Pi_{1}(X) \rightarrow \mathcal{V}$.

We denote by $\operatorname{Sh}(X, \mathcal{V})$ the category of sheaves on $X$ with values in $\mathcal{V}$. It is again a Grothendieck abelian category. By $D^{b}(\operatorname{Sh}(X))$ we denote the bounded derived category of $\operatorname{Sh}(X, \mathcal{V})$.

Further, let $(X, S)$ be a stratified complex manifold. Then, as in $\S 1.1$, we have: 
- The abelian category $\operatorname{Sh}(X, S ; \mathcal{V})$ of $S$-constructible sheaves on $X$ with values in $\mathcal{V}$.

- The triangulated category $D^{b}(X, S ; \mathcal{V})$ of $S$-constructible complexes on $X$ with values in $\mathcal{V}$.

- The abelian subcategory $\operatorname{Perv}(X, S ; \mathcal{V}) \subset D^{b}(X, S ; \mathcal{V})$.

B. Finiteness issues: subanalytic topology. The requirement for $\mathcal{V}$ to be a Grothendieck category is, in general, forced by the necessity to have infinite products as in (1.4.1). This excludes, for instance, the case $\mathcal{V}=$ Vect $_{\mathrm{k}}^{\mathrm{fd}}$ or any other abelian category with a perfect duality.

However, if we are interested only in constructible sheaves and complexes, then the following more restricted framework is sufficient.

Let $X$ be a real analytic manifold. We recall [29], [31] the concept of a subanalytic set in $X$. In particular, we have the subanalytic site $X_{\mathrm{sa}}$ of $X$. This is the poset of relatively compact subanalytic open subsets $U \subset X$ with coverings being finite coverings in the usual sense. Since all coverings are finite, we can speak about sheaves on $X_{\mathrm{sa}}$ with values in any abelian category $\mathcal{V}$, not necessarily Grothendieck one.

In particular, if $(X, S)$ is a stratified complex manifold, then we have the categories

$$
\operatorname{Sh}\left(X_{\mathrm{sa}}, S ; \mathcal{V}\right) \subset D^{b}\left(X_{\mathrm{sa}}, S ; \mathcal{V}\right) \supset \operatorname{Perv}\left(X_{\mathrm{sa}}, S ; \mathcal{V}\right) .
$$

If $\mathcal{V}$ has a perfect duality $V \mapsto V^{*}$, then $D^{b}\left(X_{\text {sa }}, S ; \mathcal{V}\right)$ and $\operatorname{Perv}\left(X_{\text {sa }}, S ; \mathcal{V}\right)$ also have a perfect duality which we call the Verdier duality and denote $\mathcal{F} \mapsto \mathbb{D} \mathcal{F}$. For an arbitrary $\mathcal{V}$ we have the Verdier duality in the form of anti-equivalences

$$
\mathbb{D}: \operatorname{Perv}\left(X_{\mathrm{sa}}, S, \mathcal{V}\right) \rightarrow \operatorname{Perv}\left(X_{\mathrm{sa}}, S, \mathcal{V}^{\mathrm{op}}\right), \quad \mathbb{D}: D^{b}\left(X_{\mathrm{sa}}, S ; \mathcal{V}\right) \rightarrow D^{b}\left(X_{\mathrm{sa}}, S ; \mathcal{V}^{\mathrm{op}}\right)
$$

Proposition 1.4.3. If $\mathcal{V}$ is a Grothendieck abelian category, then the restriction functors

$\operatorname{Sh}(X, S ; \mathcal{V}) \rightarrow \operatorname{Sh}\left(X_{\mathrm{sa}}, S ; \mathcal{V}\right), \quad D^{b}(X, S ; \mathcal{V}) \rightarrow D^{b}\left(X_{\mathrm{sa}}, S ; \mathcal{V}\right), \quad \operatorname{Perv}(X, S ; \mathcal{V}) \rightarrow \operatorname{Perv}\left(X_{\mathrm{sa}}, S ; \mathcal{V}\right)$ are equivalences of categories.

Proof: Same argument as in [31], §7.1.

C. Conventions for the rest of the paper. (1) In the sequel we will drop the subscript "sa" and simply speak about $S$-constructible sheaves and complexes on $X$ with values in $\mathcal{V}$, while having in mind the subanalytic topology. In particular, we will use freely the standard functorialities such as $f_{*}, f^{*}, f_{!}, f_{!}$on constructible complexes on $X_{\mathrm{sa}}$, see [31], $\S 7.1$ for their construction. For a holomorphic function $f$, we will also use the vanishing cycle functor $\Phi_{f}$ as well as the fact that it preserves $\mathcal{V}$-valued perverse sheaves and commutes with Verdier duality in the form (1.4.2).

(2) We will freely use the (obvious) analogs of the concepts and results of $\S \S 1.1-1.3$ for perverse sheaves with values in $\mathcal{V}$. In particular, we will use the concept of a pure skeleton, 
of a self-dual skeleton (either when $\mathcal{V}$ has a perfect duality or with respect to (1.4.2)), as well as both the $(\Phi, \Psi)$-description and the Dirac descriprion of $\mathcal{V}$-valued perverse sheaves on a disk in terms of diagrams formed by objects and morphisms of $\mathcal{V}$. In the same vein, we will use Proposition 1.3.3(a) in the $\mathcal{V}$-valued context.

\section{Bisheaves and bialgebras: perverse sheaves on sym- metric products}

\subsection{The symmetric product $\operatorname{Sym}^{n}(\mathbb{C})$, its complex strata and real skeleton}

A. The complex strata. We denote by $\Sigma_{n}$ the symmetric group of order $n$. Fix $n \geqslant 1$ and let $X=\operatorname{Sym}^{n}(\mathbb{C})=\mathbb{C}^{n} / \Sigma_{n}$ be the $n$th symmetric product of $\mathbb{C}$. As well known, it can be seen in either of two ways:

(1) As the space of effective divisors of degree $n$, i.e., of formal sums

$$
\sum \lambda_{i} x_{i}, \quad \lambda_{i} \in \mathbb{Z}_{>0}, \quad x_{i} \in \mathbb{C}, \quad \sum \lambda_{i}=n
$$

(2) As the space of monic polynomials of degree $n$

$$
f(x)=x^{n}+a_{n-1} x^{n-1}+\cdots+a_{0}, \quad a_{i} \in \mathbb{C} .
$$

Explicitly, $x_{i}$ in (1) is a root of $f(x)$ with multiplicity $\lambda_{i}$. The description (2) makes it clear that $\operatorname{Sym}^{n}(\mathbb{C})$ is isomorphic to $\mathbb{C}^{n}$. The description (1) gives a natural Whitney stratification of $\operatorname{Sym}^{n}(\mathbb{C})$ by the type of divisors. It is labelled by (unordered) partitions of $n$, i.e., by sequences

$$
\lambda=\left(\lambda_{1} \geqslant \cdots \geqslant \lambda_{p}>0\right), \quad|\lambda|:=\sum \lambda_{i}=n .
$$

Here we can think of $\lambda$ as a Young diagram with $n$ cells. We denote the set of such partitions (or Young diagrams) by $\mathcal{P}(n)$. For $\lambda \in \mathcal{P}(n)$ we denote $l(\lambda)=p$ the number of parts of $\lambda$, i.e., the number of rows in the Young diagram.

We denote by $\leqslant$ the standard partial ordering on $\mathcal{P}(n)$ whereby $\lambda \leqslant \mu$ if and only if $\mu$ is obtained from $\lambda$ by moving some cells to the right and up. With respect to this order, the 1 -part partition $(n)$ is the minimal one while the partition $(1, \cdots, 1)$ is the maximal one.

By definition, the stratum $X_{\lambda}^{\mathbb{C}}$ associated to $\lambda$ consists of divisors that can be represented as $\sum \lambda_{i} x_{i}$ with all $x_{i}$ distinct. For example, $X_{1, \cdots, 1}^{\mathbb{C}}$ is the open stratum (all points distinct), while $X_{n}^{\mathbb{C}} \simeq \mathbb{C}$ is the minimal stratum (all points coincide). The following is straightforward.

Proposition 2.1.1. (a) $X_{\lambda}^{\mathbb{C}}$ is a smooth locally closed subvariety in $\operatorname{Sym}^{n}(\mathbb{C})$ of dimension $l(\lambda)$. The collection $S=\left\{X_{\lambda}^{\mathbb{C}}\right\}$ forms a Whitney stratification of $X=\operatorname{Sym}^{n}(\mathbb{C})$.

(b) Fo $\lambda, \mu \in \mathcal{P}(n)$ we have $X_{\lambda}^{\mathbb{C}} \subset \bar{X}_{\mu}^{\mathbb{C}}$ if and only if $\lambda \leqslant \mu$. 
We will refer to $S$ as the diagonal stratification and use the notation $S_{n}$ to empahize the dependence on $n$, if needed. We will be interested in the category $\operatorname{Perv}\left(\operatorname{Sym}^{n}(\mathbb{C}), S\right)$.

Note that the group structure on $\mathbb{C}$ gives a morphism

$$
\sigma: \operatorname{Sym}^{n}(\mathbb{C}) \longrightarrow \mathbb{C}, \quad \sum \lambda_{i} x_{i} \mapsto \sum^{\mathbb{C}} \lambda_{i} x_{i}
$$

where $\sum^{\mathbb{C}}$ is the operation of addition in $\mathbb{C}$. We denote $\operatorname{Sym}_{0}^{n}(\mathbb{C})$ the preimage of 0 under $\sigma$. It is identified with the space of monic polynomials $f(x)$ with $a_{n-1}=0$. Note that we have an identification

$$
\operatorname{Sym}^{n}(\mathbb{C}) \simeq \mathbb{C} \times \operatorname{Sym}_{0}^{n}(\mathbb{C}) .
$$

It is given by the action of $\mathbb{C}$ on $\operatorname{Sym}^{n}(\mathbb{C})$ by additive shifts $f(x) \mapsto f(x+c)$. Each stratum $X_{\lambda}$ is the product of $\mathbb{C}$ and the induced stratum in $\operatorname{Sym}_{0}^{n}(\mathbb{C})$.

B. The real skeleton. Let $K=\operatorname{Sym}^{n}(\mathbb{R}) \subset \operatorname{Sym}^{n}(\mathbb{C})$ be the $n$th symmetric product of $\mathbb{R}$, i.e., the subspace formed by effective divisors consisting of real points. As in the complex case, we have the action of $\mathbb{R}$ on $\operatorname{Sym}^{n}(\mathbb{R})$ given by adding the same number to all the points of a divisor. This action induces identifications

$$
\begin{gathered}
\operatorname{Sym}^{n}(\mathbb{R}) \simeq \mathbb{R} \times \operatorname{Sym}_{0}^{n}(\mathbb{R}), \\
\operatorname{Sym}^{n}(\mathbb{R}) / \mathbb{R} \simeq \operatorname{Sym}_{0}^{n}(\mathbb{R}) .
\end{gathered}
$$

Alternatively, $\operatorname{Sym}^{n}(\mathbb{R})$ can be seen as the set of those monic polynomials whose all roots are real, and $\operatorname{Sym}_{0}^{n}(\mathbb{R})$ as the set of monic polynomial with all roots real and summing to 0 . So $\operatorname{Sym}^{n}(\mathbb{R})$ is a closed "curvilinear cone" in the Euclidean space $\mathbb{R}^{n}$ formed by all monic polynomials with real coefficients.

Similarly, let $\mathbb{R}_{0}^{n} \simeq \mathbb{R}^{n-1}$ be the Euclidean space of all of monic polynomials with real coefficients and with $a_{1}=0$. Then $\operatorname{Sym}_{0}^{n}(\mathbb{R})$ is a "curvilinear cone" in $\mathbb{R}_{0}^{n}$. In examples, it is convenient to visualize $\operatorname{Sym}_{0}^{n}(\mathbb{R})$ instead of $\operatorname{Sym}^{n}(\mathbb{R})$, factoring out the translation symmetry.

Examples 2.1.4. (a) Let $n=2$. Then $\mathbb{R}_{0}^{2}=\mathbb{R}$ is the real line formed by polynomials $x^{2}+a=0$, and $\operatorname{Sym}_{0}^{2}(\mathbb{R})=\{a \leqslant 0\}$ is the negative real line.

(b) Let $n=3$. Then $\mathbb{R}_{0}^{3}$ consists of real cubic polynomials of the form $x^{3}+a x+b$, and

$$
\operatorname{Sym}_{0}^{3}(\mathbb{R})=\left\{(a, b) \mid 4 a^{3}+27 b^{2} \leqslant 0\right\}
$$

is the interior of the real semi-cubical parabola, see Fig. 3.

We note that $K=\operatorname{Sym}^{n}(\mathbb{R})$ has a natural stratification $S_{K}$ labelled by ordered partitions of $n$, i.e., sequences

$$
\alpha=\left(\alpha_{1}, \cdots, \alpha_{p}\right), \quad \alpha_{i} \in \mathbb{Z}_{>0}, \quad \sum \alpha_{i}=n .
$$

We denote $l(\alpha)=p$ the length of $\alpha$, and by $\bar{\alpha}=\left(\alpha_{\sigma(1)} \geqslant \cdots \geqslant \alpha_{\sigma(p)}\right)$ (for appropriate $\left.\sigma \in S_{p}\right)$ the ordered partition corresponding to $\alpha$. 
The stratum $K_{\alpha}=\operatorname{Sym}_{\alpha}^{n}(\mathbb{R})$ corresponding to $\alpha$, consists of divisors of the form

$$
\alpha_{1} x_{1}+\cdots+\alpha_{p} x_{p}, \quad x_{i} \in \mathbb{R}, \quad x_{1}<\cdots<x_{p} .
$$

The number of ordered partitions of $n$ is $2^{n-1}$, they are in bijection with arbitrary subsets of the set of plus signs in the equality

$$
n=1+1+1+\cdots+1 \text {. }
$$

Conceptually, these plus signs correspond to simple roots of the root system $A_{n-1}$. To get $\alpha$ we simply perform the additions corresponding to the chosen subset of the plus signs. We therefore denote the set of ordered partitions of $n$ by $2^{n-1}$ and equip it with the partial order of reverse inclusion of subsets. Geometrically, we view $\mathbf{2}^{n-1}$ as the set of vertices of an $(n-1)$-dimensional cube.

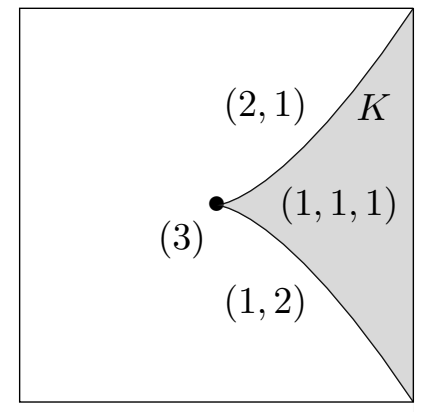

Figure 3: The real skeleton $K=K_{3}$ in $\operatorname{Sym}_{0}^{3}(\mathbb{C})$ and its strata labelled by $2^{3-1}$.

Further, given $\alpha \in \mathbf{2}^{n-1}$, we denote by $\alpha^{\vee}$ the complementary ordered partition, for which the corresponding subset in $\{1, \cdots, n-1\}$ are complementary. Thus, for example, $(1,1,1)^{\vee}=(3)$ etc. Note that the un-ordered partition $\overline{\alpha^{\vee}}$ corresponds to the transpose of the Young diagram corresponding to the un-ordered partition $\alpha$. 
Example 2.1.6. Let $n=3$. The poset $\mathbf{2}^{3-1}$, with order indicated by arrows, has the form

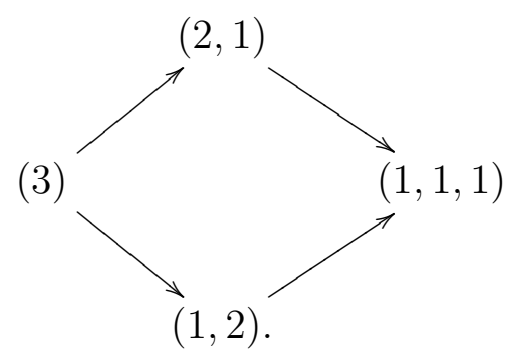

Proposition 2.1.7. For each $\alpha \in \mathbf{2}^{n-1}$, the stratum $K_{\alpha}$ is a cell of real dimension $l(\alpha)$. We have $K_{\alpha} \subset \bar{K}_{\beta}$ if and only if $\alpha \leqslant \beta$ in $\mathbf{2}^{n-1}$. The image of $K_{\alpha}$ under the embedding $\operatorname{Sym}^{n}(\mathbb{R}) \subset \operatorname{Sym}^{n}(\mathbb{C})$ lies in the stratum $X_{\bar{\alpha}}$.

\subsection{Imaginary strata and fine cells}

A. The imaginary strata of $\operatorname{Sym}^{n}(\mathbb{C})$. The imaginary part map $\operatorname{Im}: \mathbb{C} \rightarrow \mathbb{R}$ gives a mapping

$$
\mathfrak{I}: \operatorname{Sym}^{n}(\mathbb{C}) \rightarrow \operatorname{Sym}^{n}(\mathbb{R})=K, \quad \mathbf{z}=\sum \lambda_{i} z_{i} \mapsto \sum \lambda_{i} \operatorname{Im}\left(z_{i}\right) .
$$

The fibers of $\mathfrak{I}$ are totally real subanalytic subspaces (not necessarily submanifolds) of $\operatorname{Sym}^{n}(\mathbb{C})$.

We call the imaginary stratification of $\operatorname{Sym}^{n}(\mathbb{C})$ the pullback, under $\mathfrak{I}$, of the stratification $\left(K_{\alpha}\right)$ of $K$ and denote the corresponding strata, as well as their embeddings, by

$$
X_{\alpha}^{\mathfrak{I}}=\mathfrak{I}^{-1}\left(K_{\alpha}\right), \quad j_{\alpha}: X_{\alpha}^{\operatorname{Im}} \hookrightarrow X=\operatorname{Sym}^{n}(\mathbb{C}), \quad \alpha \in \mathbf{2}^{n-1} .
$$

Explicitly, a point (written as a diivisor) $\mathbf{z} \in \operatorname{Sym}^{n}(\mathbb{C})$ lying in $X_{\alpha}^{\operatorname{Im}}$ splits (in a unique way) into a sum of effective divisors $\mathbf{z}_{1}+\cdots+\mathbf{z}_{p}$ such that

$$
\mathfrak{I}\left(\mathbf{z}_{1}\right)=\alpha_{1} \cdot y_{1}, \cdots, \quad \mathfrak{I}\left(\mathbf{z}_{p}\right)=\alpha_{p} \cdot y_{p}, \quad y_{1}<\cdots<y_{p},
$$

see Fig. 4.

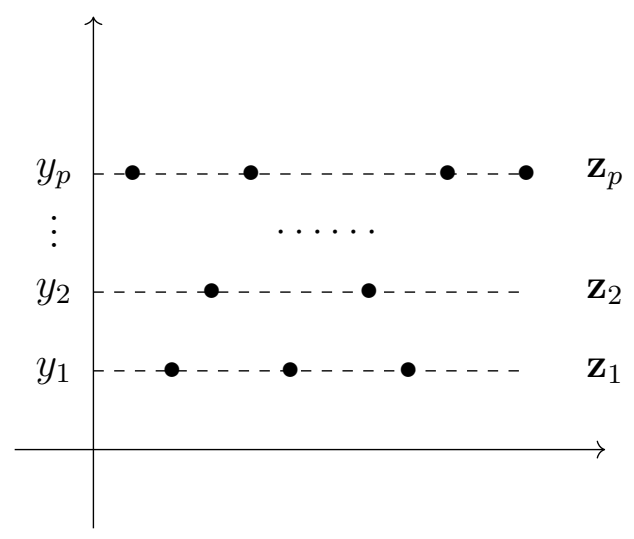

Figure 4: A point $\mathbf{z}$ of $X_{\alpha}^{\operatorname{Im}}$. 
We also have the similar real part map

$$
\left.\mathfrak{R}: \operatorname{Sym}^{m} \mathbb{C}\right) \rightarrow \operatorname{Sym}^{m}(\mathbb{R}), \quad m \geqslant 0
$$

Applying it to the divisor $\mathbf{z}_{\nu}$ corresponding to $\mathbf{z}$ as above gives a map $\rho_{\alpha, \nu}: X_{\alpha}^{\mathfrak{I}} \rightarrow \operatorname{Sym}^{\alpha_{\nu}}(\mathbb{R})$, $\nu=1, \cdots, p$. These maps combine into a projection

$$
\rho_{\alpha}=\left(\rho_{\alpha, \nu}\right)_{\nu=1}^{p}: X_{\alpha}^{\mathfrak{I}} \longrightarrow \prod_{\nu=1}^{p} \operatorname{Sym}^{\alpha_{\nu}}(\mathbb{R}), \quad \rho_{\alpha, \nu}(\mathbf{z})=\mathfrak{R}\left(\mathbf{z}_{\nu}\right)
$$

and the following is clear.

Proposition 2.2.3. $\rho_{\alpha}$ is a locally trivial fibration with fibers homeomorphic to $\mathbb{R}^{l(\alpha)}$. In particular, $X_{\alpha}^{\mathfrak{I}}$ is a contractible $C W$-complex of pure real dimension $n+l(\alpha)$.

Examples 2.2.4. (a) If $\alpha=(n)$ is the partition with one part, then $X_{(n)}^{\operatorname{Im}}$ consists of $\left\{z_{1}, \cdots, z_{n}\right\}$ with $\operatorname{Im}\left(z_{1}\right)=\cdots=\operatorname{Im}\left(z_{n}\right)$ and so is identified with $\mathbb{R} \times \operatorname{Sym}^{n}(\mathbb{R})$.

(b) Note that $\mathfrak{I}$ preserves the total sum of the points of a divisor so induces a map

$$
\mathfrak{I}_{0}: \operatorname{Sym}_{0}^{n}(\mathbb{C}) \longrightarrow \operatorname{Sym}_{0}^{n}(\mathbb{R})
$$

Consider $n=2$. After undertifying $\operatorname{Sym}_{0}^{2}(\mathbb{C})$ with $\mathbb{C}$ and $\operatorname{Sym}_{0}^{2}(\mathbb{R})$ with $\mathbb{R}_{\geqslant 0}$ we get the map

$$
\mathbb{C} \longrightarrow \mathbb{R}_{\geqslant 0}, \quad z \mapsto(\operatorname{Im} \sqrt{z})^{2}
$$

The preimage of 0 under this map is the positive line $\mathbb{R}_{\geqslant 0}$ and the other preimages are parabolas positioned horizontally (so the positive line is the "infinitely sharp parabola"). the induced imaginary strata on $\operatorname{Sym}_{0}^{2}(\mathbb{C})=\mathbb{C}$ are the positive real line and its complement:

$$
S_{2}^{\operatorname{Im}} \cap \operatorname{Sym}_{0}^{2}(\mathbb{C})=\mathbb{R}_{\geqslant 0}, \quad S_{1,1}^{\operatorname{Im}} \cap \operatorname{Sym}_{0}^{2}(\mathbb{C})=\mathbb{C}-\mathbb{R}_{\geqslant 0}
$$

B. The fine cells. We will further consider the preimages, under $\rho_{\alpha}$, of the product strata of $\prod_{\nu=1}^{p} \operatorname{Sym}^{\alpha_{\nu}}(\mathbb{R})$ and call these preimages the fine cells. From this point of view, fine cells are labelled by pairs $(\alpha, \Gamma)$, where $\alpha=\left(\alpha_{1}, \cdots, \alpha_{p}\right) \in \mathbf{2}^{n-1}$ and $\Gamma=\left(\gamma^{(1)}, \cdots, \gamma^{(p)}\right)$ is a sequence of ordered partitions, with $\gamma^{(\nu)} \in \mathbf{2}^{\alpha_{\nu}-1}$.

Note that for a given $\alpha$, a datum of $\Gamma$ as above is equivalent to a datum of a single partition $\gamma \in \mathbf{2}^{n-1}$ refining $\alpha$. This $\gamma$ is obtained simply by writing all the parts of all the $\gamma^{(i)}$ together in a single sequence. Thus, a fine cell is labelled by a pair $\alpha \leqslant \gamma$ of ordered partitions of $n$, and will be denoted $X_{[\alpha: \gamma]}$. Thus

$$
X_{\alpha}^{\mathfrak{I}}=\bigsqcup_{\gamma \geqslant \alpha} X_{[\alpha: \gamma]}
$$


Proposition 2.2.5. (a) $X_{[\alpha: \gamma]}$ is a topological cell (space homeomorphic to an open ball) of dimension $l(\alpha)+l(\gamma)$.

(b) The collection of the $X_{[\alpha: \gamma]}, \alpha \leqslant \gamma$, forms a cell decomposition (stratification into cells) of $\operatorname{Sym}^{n}(\mathbb{C})$ refining the complex stratification $S$. More precisely, let $\lambda \in \mathcal{P}(n)$ is an unordered partition of $n$. The complex stratum $X_{\lambda}^{\mathbb{C}}$ is the following union of fine cells:

$$
X_{\lambda}^{\mathbb{C}}=\bigsqcup_{\substack{\alpha \leq \gamma \\ \gamma=\lambda}} X_{[\alpha: \gamma]} .
$$

(c) We have $X_{[\alpha: \gamma]} \subset \overline{X_{\left[\alpha^{\prime}: \gamma^{\prime}\right]}}$ if and only if $\alpha \leqslant \alpha^{\prime}$ and $\gamma \leqslant \gamma^{\prime}$ in $\mathbf{2}^{n-1}$.

(d) In addition, for any $\alpha \in \mathbf{2}^{n-1}$ and $\lambda \in \mathcal{P}(n)$ we have

$$
X_{\alpha}^{\mathfrak{I}} \cap X_{\lambda}^{\mathbb{C}}=\bigsqcup_{\substack{\gamma=\lambda \\ \alpha \leqslant \gamma}} X_{[\alpha: \gamma]} .
$$

Proof: Proposition 2.2.3 implies part (a). The other parts are straightforward.

We denote the stratification into fine cells by $S^{\text {fine }}$.

Examples 2.2.6. (a) For $n=2$ the stratification induced by $S^{\text {fine }}$ on $\operatorname{Sym}_{0}^{2}(\mathbb{C})=\mathbb{C}$ is the cell decomposition consisting of the point 0 , the open half line $\mathbb{R}_{>0}$ and the complement $\mathbb{C} \backslash \mathbb{R}_{\geqslant 0}$. This example illustrates the fact that the cell decomposition $S^{\text {fine }}$ is not regular: the closure of one cell can approach a lower dimensional cell from more than one direction.

(b) The open complex stratum $\operatorname{Sym}_{\neq}^{n}(\mathbb{C})=X_{1^{n}}^{\mathbb{C}} \subset \operatorname{Sym}^{n}(\mathbb{C})$ is the union of fine cells $X_{\left[\alpha: 1^{n}\right]}$ for all $\alpha=\left(\alpha_{1}, \cdots, \alpha_{p}\right) \in \mathbf{2}^{n-1}$. This is the classical Fox-Neuwirth cell decomposition of $\operatorname{Sym}_{\neq}^{n}(\mathbb{C})$, see [18] [19] used for computing the cohomology of the braid group $\operatorname{Br}_{n}=$ $\pi_{1}\left(\operatorname{Sym}_{\neq}^{n}(\mathbb{C})\right)$.

\subsection{Perverse sheaves on $\operatorname{Sym}^{n}(\mathbb{C})$ : generalities}

Let $\mathcal{V}$ be an abelian category. For each $n \geqslant 1$ we consider the category $\operatorname{Perv}\left(\operatorname{Sym}^{n}(\mathbb{C}), S, \mathcal{V}\right)$ of $\mathcal{V}$-valued perverse sheaves on $\operatorname{Sym}^{n}(\mathbb{C})$ smooth with respect to the diagklnal stratification. An elementary description of this category is known for $n \leqslant 3$. The case $n=1$ is trivial (the objects are constant sheaves), and the cases $n=2,3$ are recalled in Ch. 5. In this section we collect several general remarks.

\section{A. Purity of the skeleton.}

Proposition 2.3.1. The skeleton $K=\operatorname{Sym}^{n}(\mathbb{R})$ is pure and self-dual for $\left(X=\operatorname{Sym}^{n}(\mathbb{C}), S\right)$ with respect to perverse sheaves with values in any category $\mathcal{V}$. 
To prove this, note that we have the diagram

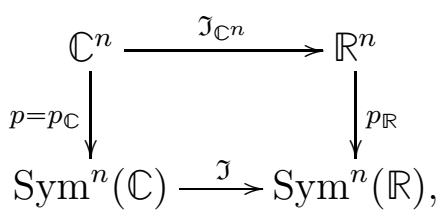

where the vertical arrows are the maps of taking the quotient by $\Sigma_{n}$. Inside $\mathbb{C}^{n}$, we have the root arrangement (for the Lie algebra $\mathfrak{g l}_{n}(\mathbb{C})$ )

$$
\mathcal{H}_{\mathbb{C}}=\bigcup_{0 \leqslant i<j \leqslant n} H_{i j}^{\mathbb{\complement}}, \quad H_{i j}^{\mathbb{\complement}}=\left\{\left(z_{1}, \cdots, z_{n}\right) \mid z_{i}=z_{j}\right\} .
$$

It is the complexification of the similar arrangement $\mathcal{H}$ in $\mathbb{R}^{n}$.

Proposition 2.3.3. Suppose $\mathcal{F} \in \operatorname{Perv}\left(\operatorname{Sym}^{n}(\mathbb{C}), S, \mathcal{V}\right)$. Then:

(a) $p^{*} \mathcal{F} \in \operatorname{Perv}\left(\mathbb{C}^{n}, \mathcal{H}, \mathcal{V}\right)$

(b) $\mathcal{F}=\left(p_{*} p^{*} \mathcal{F}\right)^{\Sigma_{n}}$

Proposition 2.3.3 implies that we can embed $\operatorname{Perv}\left(\operatorname{Sym}^{n}(\mathbb{C}), S, \mathcal{V}\right)$ into the category of $\Sigma_{n}$-eqiivariant perverse sheaves on $\left(\mathbb{C}^{n}, \mathcal{H}\right)$. Objects of the latter category can, in principle, be analyzed by the methods of [32] [60]. In this paper we use a different approach, intrinsic to $\operatorname{Sym}^{n}(\mathbb{C})$. It has the advantage of involving linear algebra data of much smaller size and can be seen as a generalization of the most classical $(\Phi, \Psi)$-description of $\operatorname{Perv}(\mathbb{C}, 0)$ (Example 1.1.5).

Proof of Proposition 2.3.3: (a) Because the strata of $S$ are the images of the strata of $S_{\mathcal{H}}$, we have that $p * \mathcal{F}$ is an $S_{\mathcal{H}}$-constructible complex. Let us verify the perversity condition $\left(P^{+}\right)$for $p^{*} \mathcal{F}$. Since $p$ preserves the dimensions of the strata, the condition follows from the same condition for $\mathcal{F}$. The condition $\left(P^{-}\right)$for $p^{*} \mathcal{F}$ is equivalent to $\left(P^{+}\right)$for the Verdier dual $\mathbb{D}\left(p^{*} \mathcal{F}\right)$, which is identified with $p^{*}(\mathbb{D} \mathcal{F})$. So $\left(P^{-}\right)$for $p^{*} \mathcal{F}$ follows from $\left(P^{+}\right)$for $\mathbb{D} \mathcal{F}$, i.e., from $\left(P^{-}\right)$for $\mathcal{F}$. Here $\mathbb{D}$ is understood in the sense of (1.4.2).

Part (b) is clear.

Proof of Proposition 2.3.1. Proposition 2.3.3 implies that

$$
\underline{R \Gamma}_{K} \mathcal{F}=\left(p_{*} \underline{R}_{\mathbb{R}^{n}}\left(p^{*} \mathcal{F}\right)\right)^{\Sigma_{n}},
$$

and so our statement follows from Proposition 1.3.3(a).

B. The total vanishing cycle functor. For $\mathcal{F} \in \operatorname{Perv}\left(\operatorname{Sym}^{n}(\mathbb{C}), S, \mathcal{V}\right)$ we denote

$$
\Phi_{\text {tot }}(\mathcal{F})=R_{K}(\mathcal{F})_{0} \in \mathrm{Ob}(\mathcal{V})
$$

the stalk at $0 \in K$ of the sheaf $R_{K}(\mathcal{F})$ and call it the total vanishing cycle space of $\mathcal{F}$.

Recall that the coefficients $a_{1}, \cdots, a_{n}$ of an indeterminate monic polynomial form a coordinate system on $\operatorname{Sym}^{n}(\mathbb{C})$, and $\operatorname{Sym}_{0}^{n}(\mathbb{C})$ is defined by $a_{1}=0$. 


\section{Proposition 2.3.6.}

$$
\Phi_{\text {tot }}(\mathcal{F}) \simeq \Phi_{a_{2}}\left(\left.\mathcal{F}\right|_{\operatorname{Sym}_{0}^{n}(\mathbb{C})}\right)_{0}
$$

is identified with the stalk at 0 of the perverse sheaf of vanishing cycles of the restriction $\left.\mathcal{F}\right|_{\operatorname{Sym}_{0}^{n}(\mathbb{C})}$ with respect to the linear function $a_{2}$.

Proof: Under the projection $p: \mathbb{C}^{n} \rightarrow \operatorname{Sym}^{n}(\mathbb{C})$ the coordinates $a_{m}$ are the elementary symmetric functions of the standard coordinates $x_{1}, \cdots, x_{n}$ on $\mathbb{C}^{n}$. In particular, the quadratic form $Q(x)=x_{1}^{2}+\cdots+x_{n}^{2}$ descends to the function $a_{1}^{2}-2 a_{2}$ on $\operatorname{Sym}^{n}(\mathbb{C})$. So by $(2.3 .4)$ and $(1.3 .4)$

$$
\Phi_{\text {tot }}(\mathcal{F}) \simeq \Phi_{Q}\left(p^{*} \mathcal{F}\right)_{0}^{\Sigma_{n}}=\Phi_{a_{1}^{2}-2 a_{2}}(\mathcal{F})_{0}=\Phi_{-2 a_{2}}\left(\left.\mathcal{F}\right|_{\operatorname{Sym}_{0}^{n}(\mathbb{C})}\right)_{0},
$$

as claimed.

C. The Cousin resolution. We now formulate a general scheme of analyzing objects of $\operatorname{Perv}\left(\operatorname{Sym}^{n}(\mathbb{C}), S, \mathcal{V}\right)$ which is similar in spirit to the one used for hyperplane arrangements in [32] but is intrinsic to $\operatorname{Sym}^{n}(\mathbb{C})$. We fix a k-linear abelian category $\mathcal{V}$. Recall that $j_{\alpha}: X_{\alpha}^{\mathfrak{I}} \rightarrow \operatorname{Sym}^{n}(\mathbb{C})$ is the embedding of the imaginary stratum corresponding to $\alpha \in \mathbf{2}^{n-1}$.

Proposition 2.3.7. Let $\mathcal{F} \in \operatorname{Perv}\left(\operatorname{Sym}^{n}(\mathbb{C}), S, \mathcal{V}\right)$.

(a) For any $\alpha \in \mathbf{2}^{n-1}$ the complex $j_{\alpha}^{!} \mathcal{F}$ on $X_{\alpha}^{\operatorname{Im}}$ is quasi-isomorphic to a single sheaf in degree equal to $-l(\alpha)$.

(b) Denoting the sheaf in (a) by $\widetilde{\mathcal{E}}_{\alpha}=\widetilde{\mathcal{E}}_{\alpha}(\mathcal{F})$, the complex $R j_{\alpha *} \widetilde{\mathcal{E}}_{\alpha}$ reduces to a single sheaf $\mathcal{E}_{\alpha}=\mathcal{E}_{\alpha}(\mathcal{F})=R^{0} j_{\alpha *} j_{\alpha}^{!} \mathcal{F}$. This sheaf is constant on each fine cell.

(c) $\mathcal{F}$ has an explicit representative (the Cousin resoluton) of the form

$$
\mathcal{E}^{\bullet}(\mathcal{F})=\left\{\mathcal{E}_{(1, \cdots, 1)}(\mathcal{F}) \stackrel{\text { }}{\rightarrow} \bigoplus_{l(\alpha)=n-1} \mathcal{E}_{\alpha}(\mathcal{F}) \stackrel{\delta}{\rightarrow} \ldots \stackrel{\delta}{\rightarrow} \mathcal{E}_{(n)}(\mathcal{F})\right\} .
$$

The grading of this complex is such that $\mathcal{E}_{n}(\mathcal{F})$ is situated in degree 0 .

Proof: (a) Suppose first that $\alpha=(n)$, so $X_{\alpha}^{\mathfrak{I}}$ consists of divisors whose all components have the same imaginary part. In this case our statement is essentially equivalent to Proposition 2.3.1: indeed, $K$ consists of divisors whose all components have the same imaginary part which is, moreover, equal to 0 . So we establish the claim for $\alpha=(n)$. Let now $\alpha=$ $\left(\alpha_{1}, \cdots, \alpha_{p}\right)$ be arbitrary and suppose $\mathbf{z} \in X_{\alpha}^{\mathfrak{I}}$ is any point. Let us write $\mathbf{z}=\mathbf{z}_{1}+\cdots+\mathbf{z}_{p}$ as in (2.2.1). Then, near $\mathbf{z}$, the pair $\left(X_{\alpha}^{\mathfrak{I}}, \operatorname{Sym}^{n}(\mathbb{C})\right)$ is homeomorphic to the product of the pairs $\left(X_{\left(\alpha_{i}\right)}^{\mathfrak{S}}, \operatorname{Sym}^{\alpha_{i}}(\mathbb{C})\right)$, and so our claim follows.

(b) Let $\mathbf{z} \in \operatorname{Sym}^{n}(\mathbb{C})$. The stalk at $\mathbf{z}$ of $R^{q} j_{*} \widetilde{\mathcal{E}}^{\alpha}(\mathcal{F})$ is $H^{q}\left(U \cap X_{\alpha}^{\mathfrak{I}}, \widetilde{\mathcal{E}}^{\alpha}(\mathcal{F})\right)$, where $U$ is a small ball around $\mathbf{z}$ in $\operatorname{Sym}^{n}(\mathbb{C})$. We note that the imaginary strata $X_{\alpha}^{\mathfrak{J}}$ are the images, under $p$, of the tube cells $\mathbb{R}^{n}+i C$ where $C$ runs over the cells of the real arrangement $\mathcal{H}_{\mathbb{R}}$, see [32] , §3C. Therefore $U \cap X_{\alpha}^{\mathfrak{I}}$ is the disjoint union of the images, under $p$, of the intersections 
of balls in $\mathbb{C}^{n}$ with the tube cells and $H^{q}$ of (the pullback of) $\widetilde{\mathcal{E}}^{\alpha}(\mathcal{F})$ over such intersections vanish for $q>0$.

(c) This is a general formal consequence of (a) and (b) and of a spectral sequence (Postnikov system) corresponding to any complex of sheaves on any filtered topological space, see, e.g., [32] §1B.

\subsection{Expected answer: bar-construction for bialgebras}

A. Representations and double representations of $2^{n-1}$. We retain the notation of $\S 2.1$.

Definition 2.4.1. Let $\mathcal{V}$ be an abelian category.

(a) By a representation of $\mathbf{2}^{n-1}$ in $\mathcal{V}$ we mean a covariant functor $\gamma: \mathbf{2}^{n-1} \rightarrow \mathcal{V}$, i.e., a commutative cube of objects and morphisms

$$
\begin{gathered}
\left(E_{\alpha}, \alpha \in 2^{n-1} ; \gamma_{\alpha \alpha^{\prime}}: E_{\alpha} \longrightarrow E_{\alpha^{\prime}}, \quad \alpha \leqslant \alpha^{\prime}\right), \\
\gamma_{\alpha \alpha^{\prime \prime}}=\gamma_{\alpha^{\prime} \alpha^{\prime \prime}} \circ \gamma_{\alpha \alpha^{\prime}} \text { for } \alpha \leqslant \alpha^{\prime} \leqslant \alpha^{\prime \prime} .
\end{gathered}
$$

We denote by $\operatorname{Rep}\left(\mathbf{2}^{n-1}, \mathcal{V}\right)$ the category of representations of $\mathbf{2}^{n-1}$ in $\mathcal{V}$

(b) By an anti-representation of $\mathbf{2}^{n-1}$ in $\mathcal{V}$ we mean a contravariant functor $\delta: \mathbf{2}^{n-1} \rightarrow \mathcal{A}$, i.e., a commutative cube of objects and morphisns of the form

$$
\left(E_{\alpha}, \alpha \in 2^{n-1} ; \delta_{\alpha^{\prime} \alpha}: E_{\alpha^{\prime}} \longrightarrow E_{\alpha}, \alpha \leqslant \alpha^{\prime}\right) .
$$

(c) By a double representation of $\mathbf{2}^{n-1}$ in $\mathcal{V}$ we mean a diagram in $\mathcal{V}$

$$
\left(E_{\alpha}, \alpha \in \mathbf{2}^{n-1} ; \gamma_{\alpha \alpha^{\prime}}: E_{\alpha} \longrightarrow E_{\alpha^{\prime}}, \delta_{\alpha^{\prime} \alpha}: E_{\alpha^{\prime}} \longrightarrow E_{\alpha}, \alpha \leqslant \alpha^{\prime}\right)
$$

such that $\left(E_{\alpha}, \gamma_{\alpha \alpha^{\prime}}\right)$ is a representation of $\mathbf{2}^{n-1}$, and $\left(E_{\alpha}, \delta_{\alpha^{\prime} \alpha}\right)$ is an anti-representation. We denote by $\operatorname{Rep}^{(2)}\left(\mathbf{2}^{n-1}, \mathcal{V}\right)$ the category of double representations of $\mathbf{2}^{n-1}$ in $\mathcal{V}$.

Proposition 2.1.7 implies at once:

Corollary 2.4.2. The category $\operatorname{Sh}\left(K, S_{K}, \mathcal{V}\right)$ is identified with $\operatorname{Rep}\left(\mathbf{2}^{n-1}, \mathcal{V}\right)$.

Remarks 2.4.3. (a) Note that a representation of $2^{n-1}$ is determined by its elementary arrows

$$
\gamma_{\alpha \alpha^{\prime}}, \quad \alpha \leqslant \alpha^{\prime}, l\left(\alpha^{\prime}\right)=l(\alpha)+1
$$

which correspond to the edges of the cube. The existence of compatible $\gamma_{\alpha \alpha^{\prime}}$ for all $\alpha \leqslant$ $\alpha^{\prime}$ means that the cube is commutative in the usual sense (each 2-dimensional face is a commutative square). Similarly for anti-representations.

(b) Any representation $E=\left(E_{\alpha}, \gamma_{\alpha \alpha^{\prime}}\right)$ of $\mathbf{2}^{n-1}$ (a commutative cube) in $\mathcal{V}$ can be converted into a cochain complex in $\mathcal{V}$ by putting alternating \pm signs on the elementary arrows and summing over $\alpha$ with fixed $l(\alpha)$. We denote the complex thus obtained by

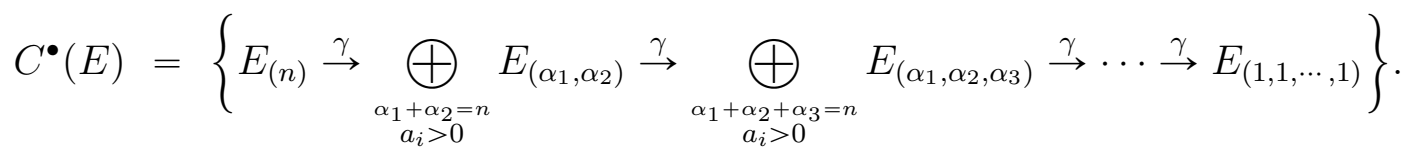


We fix its grading so that $E_{(n)}$ has degree 1 .

Similarly, any anti-representation $E=\left(E_{\alpha}, \delta_{\alpha^{\prime} \alpha}\right)$ gives rise to a chain complex $C \bullet(E)$. We can view $C \cdot(E)$ as a homological complex (differental $\delta$ lowering the degree by 1 ) with the same grading as $C \bullet(E)$. Alternatively, we can convert it into a cohomological complex by reversing the degree, that is, by putting

$$
C \cdot(E)=\left\{E_{(1,1, \cdots, 1)} \stackrel{\delta}{\rightarrow} \cdots \stackrel{\delta}{\rightarrow} \underset{\substack{\alpha_{1}+\alpha_{2}+\alpha_{3}=n \\ a_{i}>0}}{\bigoplus} E_{\left(\alpha_{1}, \alpha_{2}, \alpha_{3}\right)} \stackrel{\delta}{\rightarrow} \bigoplus_{\substack{\alpha_{1}+\alpha_{2}=n \\ a_{i}>0}} E_{\left(\alpha_{1}, \alpha_{2}\right)} \stackrel{\delta}{\rightarrow} E_{(n)}\right\} .
$$

with $E_{(n)}$ in degree $(-1)$.

A double representation $E=\left(E_{\alpha}, \gamma_{\alpha \alpha^{\prime}}, \delta_{\alpha^{\prime} \alpha}\right)$ gives therefore a complex with two differentials $\gamma$ and $\delta$, one raising, the other lowering the degrees.

B. (Anti-)representations from (co)algebras. Let $(\mathcal{V}, \otimes, \mathbf{1})$ be a $\mathbf{k}$-linear monoidal abelian category. We assume that $\otimes$ is exact in each variable.

By a connected graded algebra in $\mathcal{V}$ we mean a $\mathbb{Z}_{\geqslant 0}$-graded associative algebra $A=$ $\oplus_{n \geqslant 0} A_{n}$ in $\mathcal{V}$ with $A_{0}=\mathbf{1}$ being the unit. We denote by $\mu_{p, q}: A_{p} \otimes A_{q} \rightarrow A_{p+q}$ the components of the multiplication $\mu$ in $A$.

Given such an $A$, for each $n \geqslant 0$ we have the anti-representation $\mathbb{B}_{n}(A)$ of $\mathbf{2}^{n-1}$ called the $n$th bar-cube of $A$. Explicitly,

(1) $\mathbb{B}_{n}(A)_{\left(\alpha_{1}, \cdots, \alpha_{p}\right)}=A_{\alpha_{1}} \otimes \cdots \otimes A_{\alpha_{p}}$

(2) The elementary maps $\delta_{\alpha^{\prime} \alpha^{\prime}}$ are given by the appropriate components $\mu_{p q}$ tensored by the identities.

The commutativity of the cube follows from the associativity of $A$. For example, for $n=3$ the diagram $\mathbb{B}_{3}(A)$ has the form

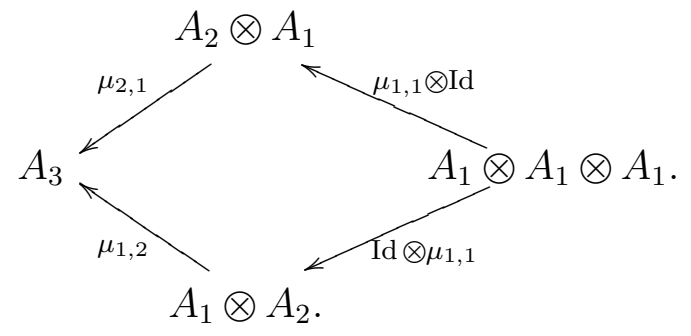

The corresponding chain complex, written as a cohomological one, will be called the $n$th bar-complex of $A$ and denoted

$$
B_{n}(A)=\left\{A_{1}^{\otimes n} \stackrel{\delta}{\rightarrow} \cdots \stackrel{\delta}{\rightarrow} \underset{\substack{\alpha_{1}+\alpha_{2}+\alpha_{3}=n \\ a_{i}>0}}{\bigoplus} A_{\alpha_{1}} \otimes A_{\alpha_{2}} \otimes A_{\alpha_{3}} \stackrel{\delta}{\rightarrow} \underset{\substack{\alpha_{1}+\alpha_{2}=n \\ a_{i}>0}}{\bigoplus} A_{\alpha_{1}} \otimes A_{\alpha_{2}} \stackrel{\delta}{\rightarrow} A_{n}\right\}
$$


its grading normalized so that $A_{n}$ is in degree $(-1)$. We note that

$$
H^{j} B_{n}(A)=\operatorname{Tor}_{-j, n}^{A}(\mathbf{1}, \mathbf{1})
$$

is the $n$th graded component of $\operatorname{Tor}_{-j}^{A}(\mathbf{1}, \mathbf{1})$ with respect to the additional grading coming from the grading of $A$.

Dually, by a graded coconnected coalgebra in $\mathcal{V}$ we mean a $\mathbb{Z}_{\geqslant 0}$-graded coassociative coalgebra $A=\bigoplus_{n \geqslant 0} A_{n}$ in $\mathcal{V}$ with $A_{0}=\mathbf{1}$ and the counit being the projection to $A_{0}=\mathbf{1}$. We denote by $\Delta_{p, q}: A_{p+q} \rightarrow A_{p} \otimes A_{q}$ the components of the comultiplication $\Delta$ in $A$. We note that

$$
\Delta_{0, n}: A_{n} \longrightarrow A_{0} \otimes A_{n}=1 \otimes A_{n}=A_{n}
$$

is the identity, and similarly for $\Delta_{n, 0}$. In particular, $A_{1}$ "consists of primitive elements", i.e., $\left.\Delta\right|_{A_{1}}=\mathbf{1} \otimes \operatorname{Id}+\mathrm{Id} \otimes \mathbf{1}$.

A graded coconnected coalgebra $A$ gives a representation $\mathbb{B}_{n}^{\dagger}(A)$ of $\mathbf{2}^{n-1}$ in $\mathcal{V}$ called the $n$th cobar-cube. Explicitly,

(1) $\mathbb{B}_{n}^{\dagger}(A)_{\left(\alpha_{1}, \cdots, \alpha_{p}\right)}=A_{\alpha_{1}} \otimes \cdots \otimes A_{\alpha_{p}}$

(2) The elementary maps $\gamma_{\alpha^{\prime} \alpha}$ are given by the appropriate components $\Delta_{p q}$ tensored by the identities.

The cochain complex of $\mathbb{B}_{n}^{\dagger}(A)$ will be called the $n$th cobar-complex of $A$ and denoted

$$
B_{n}^{\dagger}(A)=\left\{A_{n} \stackrel{\gamma}{\rightarrow} \bigoplus_{\substack{\alpha_{1}+\alpha_{2}=n \\ a_{i}>0}} A_{\alpha_{1}} \otimes A_{\alpha_{2}} \stackrel{\gamma}{\rightarrow} \underset{\substack{\alpha_{1}+\alpha_{2}+\alpha_{3}=n \\ a_{i}>0}}{\bigoplus} A_{\alpha_{1}} \otimes A_{\alpha_{2}} \otimes A_{\alpha_{3}} \stackrel{\gamma}{\rightarrow} \cdots \stackrel{\gamma}{\rightarrow} A_{1}^{\otimes n}\right\}
$$

its grading normalized so that $A_{n}$ is in degree 1 . As in the algebra case,

$$
H^{j} B_{n}^{\dagger}(A)=\operatorname{Cotor}_{A}^{j, n}(\mathbf{1}, \mathbf{1}) .
$$

C. Bialgebras and Hopf algebras in a braided monoidal category. Let $(\mathcal{V}, \otimes, \mathbf{1}, R)$ be an k-linear braided monoidal category. So for any $V_{1}, V_{2} \in \mathcal{V}$ one has the $R$-matrix

$$
R_{V_{1}, V_{2}}: V_{1} \otimes V_{2} \stackrel{\sim}{\longrightarrow} V_{2} \otimes V_{1}
$$

satisfying the braiding axioms [27]. These axioms imply, in particular, the Yang-Baxter equation for any $R_{V, V}: V \otimes V \rightarrow V \otimes V$. (Some physicists would call a braiding structure $a$ statistics.).

Recall [47] [55] that we can speak about bialgebras in $\mathcal{V}$. More precisely, if $A$ is an associative algebra in $\mathcal{V}$ with multiplication $\mu: A \otimes A \rightarrow A$, then $A \otimes A$ becomes an associative algebra with multiplication being the composition

$$
\begin{aligned}
(A \otimes A) \otimes(A \otimes A) & \simeq A \otimes(A \otimes A) \otimes A \stackrel{\operatorname{Id}_{A} \otimes R_{A, A} \otimes \operatorname{Id}_{A}}{\longrightarrow} A \otimes(A \otimes A) \otimes A \simeq \\
& \simeq(A \otimes A) \otimes(A \otimes A) \stackrel{\mu \otimes \mu}{\longrightarrow} A \otimes A .
\end{aligned}
$$


Definition 2.4.8. (a) A bialgebra in $\mathcal{V}$ is an object $A$ made into an associative algebra with unit $u: \mathbf{1} \rightarrow A$ via $\mu: A \otimes A \rightarrow A$ and into a coassociative coalgebra with counit $\varepsilon: A \rightarrow \mathbf{1}$ via $\Delta: A \rightarrow A \otimes A$ so that $\Delta$ is a morphism of algebras where $A \otimes A$ has the algebra structure (2.4.7).

(b) An antipode in a bialgebra $A$ in $\mathcal{V}$ is a morphism $\sigma: A \rightarrow A$ such that the two compositions

$$
\mu \circ(\sigma \otimes \operatorname{Id}) \circ \Delta, \quad \mu \circ(\operatorname{Id} \otimes \sigma) \circ \Delta: A \longrightarrow A
$$

are equal to $\eta \circ \varepsilon$. A bialgebra equipped with antipode is called a Hopf algebra.

We further recall the concept of the duals, see, e.g., [2] §2.1 or [55] §2. Let $\mathcal{V}$ be any monoidal category and $V, W$ be two objects. A duality datum between $V$ and $W$ is a pair of morphisms

$$
u: \mathbf{1} \longrightarrow V \otimes W, \quad c: V \otimes W \longrightarrow \mathbf{1}
$$

such that the following diagrams commute:
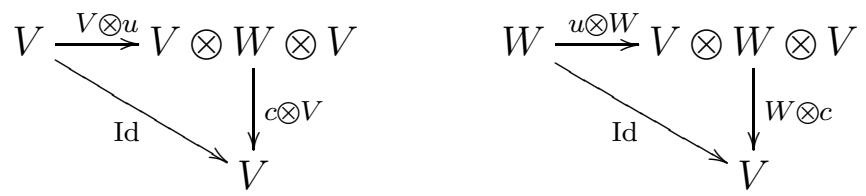

In this case $W$ is defined by $V$ uniquely up to a unique isomorphism, is called the right dual of $V$ and denoted $W=V^{*}$. Similarly $V$ is defined by $W$ uniquely up to a unique isomorphism, is called the left dual of $W$ and denoted $V={ }^{*} W$. An object having a left (resp. right) dual is called left (resp, right) dualizable.

Any monoidal category $\mathcal{V}$ can be considered as a 2-category $\Omega^{-1} \mathcal{V}$ with one object, so objects $V \in \mathcal{V}$ become 1-morphisms of $\Omega^{-1} \mathcal{V}$. The concept of left and right duals of objects in $\mathcal{V}$ becomes a particular case of the concept of the left and right adjoint of a 1-morphism of a 2-category (e.g., of the left and right adjoint of a functor between usual categories). The duality datum corresponds to the unit and counit of an adjunction.

Let us now return to the case when $\mathcal{V}$ is a braided monoidal category. In this case a left dual is also a right dual and vice versa, so we use the notation $V^{*}$ and speak simply about dualizable objects. A morphism $f: V \rightarrow W$ between dualizable objects gives the adjoint morphism $f^{t}: W^{*} \rightarrow V^{*}$. If every object of $\mathcal{V}$ is dualizable, then $\mathcal{V}$ is called rigid. In this case we can think of $V \mapsto V^{*}$ as a perfect duality on $\mathcal{V}$.

Proposition 2.4.9. Let $\mathcal{V}$ be a braided monoidal $\mathbf{k}$-linear abelian category. Let $A$ be a bialgebra $\mathcal{V}$ which is dualizable as an object of $\mathcal{V}$. Then:

(a) $A^{*}$ is also a bialgebra with $\mu_{A^{*}}=\Delta_{A}^{t}$ and $\Delta_{A^{*}}=\mu_{A}^{t}$. Further, if $A$ is a Hopf algebra, then $A^{*}$ is a Hopf algebra with antipode $\sigma_{A^{*}}=\sigma_{A}^{t}$.

(b) If $A$ is a Hopf algebra, then the antipode in $A$ is invertible.

Proof: (a) is Theorem 2.16 and (b) is a particular case of Theorem 4.1 of [55]. 
D. Primitive bialgebras. Relation to $\operatorname{Perv}\left(\operatorname{Sym}^{n}(\mathbb{C}), S\right)$. Let $\mathcal{V}$ be a braided monoidal k-linear abelian category as above.

Definition 2.4.10. A primitive bialgebra in $\mathcal{V}$ is a graded bialgebra $A=\bigoplus_{n \geqslant 0} A_{n}$ which is connected and coconnected.

For any partition $\left(\alpha_{1}, \cdots, \alpha_{k}\right) \in \mathbf{2}^{n-1}$ of $n, \alpha_{j}>0$ and a primitive bialgebra $A$, we denote

$$
\Delta_{\alpha_{1}, \cdots, \alpha_{k}}: A_{n} \longrightarrow A_{\alpha_{1}} \otimes \cdots \otimes A_{\alpha_{k}}, \quad \mu_{\alpha_{1}, \cdots, \alpha_{k}}: A_{\alpha_{1}} \otimes \cdots \otimes A_{\alpha_{k}} \longrightarrow A_{n}
$$

the corresponding components of the iterated (co)multiplication. For the trivial partition $(n)$ of $n$ we put $\Delta_{(n)}=\mu_{(n)}=\operatorname{Id}_{A_{n}}$.

Proposition 2.4.11. Let $A$ be a primitive bialgebra. For $n \geqslant 0$ define the morphism $\sigma_{n}$ : $A_{n} \rightarrow A_{n}$ by

$$
\sigma_{n}=\sum_{\left(\alpha_{1}, \cdots, \alpha_{k}\right) \in \mathbf{2}^{n-1}}(-1)^{k-1} \mu_{\alpha_{1}, \cdots, \alpha_{k}} \circ \Delta_{\alpha_{1}, \cdots, \alpha_{k}} .
$$

Then:

(a) $\sigma=\bigoplus_{n} \sigma_{n}: A \rightarrow A$ is an antipode for $A$.

(b) If each $A_{n}$ is dualzable, then $\sigma$ is invertible.

Proof: Part (a) is verified by a direct check, cf. [40] $\$ 1.2$ for a similar formula in the nonbraided case. Part (b) follows from Proposition 2.4.9, if we consider $A$ itself as a dualizable object in the braided category formed by graded objects of $\mathcal{V}$.

Given a primitive bialgebra $A$ and $n \geqslant 0$, the co-representation $\mathbb{B}_{n}(A)$ and the representation $\mathbb{B}_{n}^{\dagger}(A)$ of $\mathbf{2}^{n-1}$ combine into a double representation which we denote $\mathbb{B B}_{n}(A)$ and call the $n$th bibar-cube of $A$. For example, for $n=3$ the diagram $\mathbb{B B}_{3}(A)$ has the form

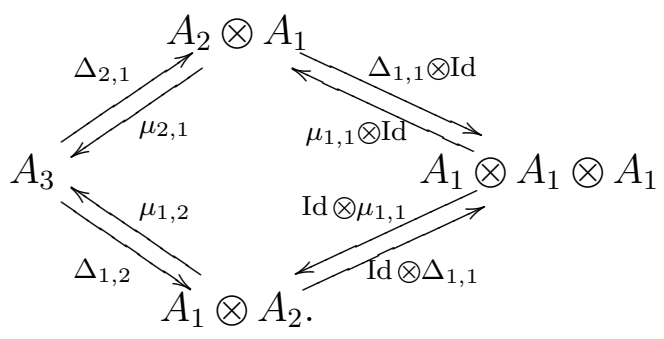

It is natural to expect that the double representation $\mathbb{B B}_{n}(A)$ actually comes from a perverse sheaf on $\operatorname{Sym}^{n}(\mathbb{C})$ smooth with respect to the diagonal factorization $S$. In the next chapter we give a precise result to this effect. 


\section{Factorizable perverse sheaves and bialgebras}

\subsection{Shuffle algebras in braided categories}

A. The tensor algebra. We return to the situation of $\S 2.4 \mathrm{C}$., that is, of a braided k-linear monoidal category $(\mathcal{V}, \otimes, \mathbf{1}, R)$. Let $V \in \mathcal{V}$; consider the tensor algebra

$$
T_{!}(V)=\bigoplus_{n=0}^{\infty} V^{\otimes n}
$$

with the multiplication

$$
V^{\otimes m} \otimes V^{\otimes n} \longrightarrow V^{\otimes m+n}
$$

begin the identity map. So as an algebra, $T !(V)$ is free. It has a unique structure of a bialgebra in $\mathcal{V}$ for which the comultiplication

$$
\Delta^{!}=\Delta^{!, R}: T_{!}(V) \longrightarrow T_{!}(V) \otimes T_{!}(V)
$$

is defined as the unique algebra map for which $T_{!}^{1}(V)=V$ is primitive, that is,

$$
\left.\Delta^{!}\right|_{T_{!}^{1}(V)}=\mathbf{1} \otimes \operatorname{Id}+\operatorname{Id} \otimes \mathbf{1}: V \longrightarrow V \otimes V .
$$

If we think of $V$ as consisting of "vectors" $x \in V$, then

$$
\Delta^{!}(x)=x \otimes 1+1 \otimes x, \quad x \in V .
$$

Since the algebra structure on $T_{!}(V) \otimes T_{!}(V)$ is defined using the $R$ matrix, see (2.4.7), the

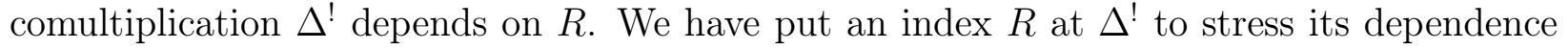
on $R$.

B. The cotensor (big shuffle) algebra. Dually, we have the cotensor algebra $T_{*}(V)$ which is a bialgebra in $\mathcal{V}$ with the same underlying object:

$$
T_{*}(V)=\bigoplus_{n=0}^{\infty} V^{\otimes n} .
$$

Its comultiplications

$$
\Delta_{m, n}=\Delta_{m, n}^{(*)}: V^{\otimes m+n} \longrightarrow V^{\otimes m} \otimes V^{\otimes n}
$$

are the identity maps, whereas the multiplication $\star_{R}$ on $T_{*}(V)$ is the so-called shuffle product, depending on $R$. That is,

$$
\star_{R}^{m, n}: V^{\otimes m} \otimes V^{\otimes n} \longrightarrow V^{\otimes m+n}
$$

acts as

$$
\star_{R}^{m, n}=\sum_{w \in \amalg_{m, n}} R_{w} .
$$


Here

$$
\amalg_{m, n}=\left\{w \in \Sigma_{m+n} \mid \text { if } i \leqslant m, j \geqslant m+1 \text { then } w(i)<w(j)\right\} \subset \Sigma_{m+n}
$$

is the subset of $(m, n)$-shuffles. The braiding axioms for $R$ imply the associativity of $\star_{R}$. Sometimes $T_{*}(V)$ is referred to as the big shuffle algebra. As a coalgebra, $T_{*}(V)$ is cofree. The following is straightforward.

Proposition 3.1.2. (a) $T_{!}(V)$ and $T_{*}(V)$ are primitive (Definition 2.4.10) bialgebras in $\mathcal{V}$. In particular, they are Hopf algebras.

(b) Let $V$ be a dualizable object of $\mathcal{V}$. Then $T_{!}(V)$ and $T_{*}(V)$ are dualizable as objects of the category of graded objects in $\mathcal{V}$ and $T_{*}(V)$ is identified with the Hopf algebra dual to $T_{!}(V)$.

C. The small shuffle (Nichols) algebra. Let us now assume, in addition, that $\mathcal{V}$ is abelian and $\otimes$ is exact in each variable.

Note that we have a canonical morphism of algebras in $\mathcal{V}$

$$
\alpha: T_{!}(V) \longrightarrow T_{*}(V)
$$

It sends the component $T_{!}^{1}(V)=V$ by the identity into the component $T_{*}^{1}(V)=V$ and is uniquely defined by this property. Indeed, $T_{!}(V)$ is freely generated by $V$, so to define its algebra morphism into anything, it is enough the specify the values on $V$ in an arbitrary way.

Proposition 3.1.3. $\alpha$ is a morphism of bialgebras.

Proof: One has to check that $\alpha$ preserves comultiplication, i.e., that

$$
\alpha \otimes \alpha \circ \Delta_{!}=\Delta_{*} \circ \alpha: T_{!}(V) \longrightarrow T_{*}(V) \otimes T_{*}(V) .
$$

Both maps are morphisms of algebras and $T_{!}(V)$ is generated as an algebra by $V=T_{!}^{1}(V)$. But on this component both maps agree. In the element notation, they both send any $x$ to $x \otimes 1+1 \otimes x$.

Definition 3.1.4. The Nichols (or small shuffle) algebra of an object $V \in \mathcal{V}$ is the bialgebra

$$
T(V)_{! *}:=\alpha\left(T_{!}(V)\right) .
$$

So it is a subalgebra of $T_{*}(V)$ and a quotient of $T_{!}(V)$.

Example 3.1.5 (abelian monodromy). Let $\Lambda \simeq \mathbb{Z}^{r}$ be a free abelian group of finite rank, and $c: \Lambda \otimes_{\mathbb{Z}} \Lambda \longrightarrow \mathbf{k}^{*}$ be a group homomorphism. If we pick a $\mathbb{Z}$-basis $e_{1}, \ldots, e_{r} \in \Lambda$, then $c$ will be determined by an $r \times r$ matrix $c_{i j}=c\left(e_{i} \otimes e_{j}\right) \in \mathbf{k}^{*}$ Consider the category Vect ${ }^{\Lambda}$ of $\Lambda$-graded vector spaces whose objects we write as

$$
V=\oplus_{\lambda \in \Lambda} V_{\lambda} .
$$


We can introduce on it a braiding given by the $R$-matrices

$$
R(x \otimes y)=c(\lambda, \mu) y \otimes x
$$

for $x \in V_{\lambda}, y \in V_{\mu}$ ("an abelian monodromy", as a physicist would say).

In particular, let $A=\left\|a_{i j}\right\|$ be a Cartan matrix and put $c_{i j}=q^{a_{i j}}$. Let $V=\mathbf{k}^{r}=\Lambda \otimes_{\mathbb{Z}} \mathbf{k}$ with its standard $\Lambda$-grading. Let $\mathfrak{g}$ be the semi-simple Lie algebra with Cartan matrix $A$. Then:

- If $q$ is not a root of 1 , then $T_{! *}(V)=U_{q}^{+}(\mathfrak{g})$ is the positive part of quantum universal enveloping algebra of $\mathfrak{g}$, see [17] [52].

- If $q$ is a root of 1 , then it was proved in [52] that $T_{! *}(V)=\mathbf{u}_{q}^{+}(\mathfrak{g})$ is the positive part of Lusztig's small (or restricted, see [43]) quantum enveloping algebra associated to $\mathfrak{g}$ and $q$. In particular, $T_{!_{*}}(V)$ is finite-dimensional.

This can be seen as an interpretation of the Lusztig's construction of $U_{q}^{+}(\mathfrak{g})\left(\right.$ resp. $\left.\mathbf{u}_{q}^{+}(\mathfrak{g})\right)$ as the quotient of a free Lie algebra by the kernel of the natural bilinear form $\langle-,-\rangle$, see [42], Ch. 1, [51]. In other words, taking the image of $\alpha$ in forming the Nichols algebra has the effect of quotienting by the kernel of $\langle-,-\rangle$, which is the universal way to encode the quantum Serre relations in $U_{q}^{+}(\mathfrak{g})$.

\subsection{Factorizable perverse sheaves}

A. The $E_{2}$-operad and braided categories. An interpretation going back to Deligne, views a braided monoidal structure in a category $\mathcal{V}$ as a datum, for each $n \geqslant 0$, of $n$-fold tensor product functors in $\mathcal{V}$ labelled by $n$-tuples of distinct points in $\mathbb{C}$. It is convenient to reformulate this approach as follows, replacing points by disks.

Let $D=\{|z| \leqslant 1\}$ be the standard unit disk in $\mathbb{C}$. We denote by $E_{2}$ the operad of little 2-disks in $\mathbb{R}^{2}=\mathbb{C}$, see [48] [13]. Thus, for each $n \geqslant 0$, the topological space $E_{2}(n)$ consists of $n$-tuples of disjoint round open disks $\left(U_{1}, \cdots, U_{n}\right)$ in $D$. Equivalently, we can see $E_{2}(n)$ as consisting of embeddings

$$
\phi=\left(\phi_{1}, \cdots, \phi_{n}\right): \bigsqcup_{i=1}^{n} D \longrightarrow D
$$

such that each $\phi_{i}: D \rightarrow D$ is a composition of a dilation and a translation. This interpretation makes manifest the operadic compositions

$$
E_{2}(k) \times E_{2}\left(n_{1}\right) \times \cdots \times E_{2}\left(n_{k}\right) \longrightarrow E_{2}\left(n_{1}+\cdots+n_{k}\right) .
$$

Thus $E_{2}$ is an operad in the category $\mathcal{T}$ op of topological spaces. Passing to fundamental groupoids, we get an operad $\Pi_{1}\left(E_{2}\right)$ in the category of groupoids. We note that each $E_{2}(n)$ is a $K(\pi, 1)$-space, so $\Pi_{1}\left(E_{2}(n)\right)$ contains all the information about its homotopy type. 
Proposition 3.2.1. Let $\mathcal{V}$ be a category. Giving a braided monoidal structure in $\mathcal{V}$ is equivalent to making $\mathcal{V}$ into a $\Pi_{1}\left(E_{2}\right)$-algebra in the category of categories, that is, defining:

(1) For any $n \geqslant 0$ and any disjoint open round disks $U_{1}, \cdots, U_{n} \subset D$, an n-variable functor

$$
\bigotimes_{\left(U_{1}, \cdots, U_{n}\right)}^{(n)}: \mathcal{V}^{n} \longrightarrow \mathcal{V}, \quad\left(V_{1}, \cdots, V_{n}\right) \mapsto \bigotimes_{\left(U_{1}, \cdots, U_{n}\right)} V_{i},
$$

(2) For any path $\gamma:[0,1] \rightarrow E_{2}(n)$, joining $\left(U_{1}, \cdots U_{n}\right)$ and $\left(U_{1}^{\prime}, \cdots U_{n}^{\prime}\right)$, a natural isomorphism of functors

$$
R_{\gamma}: \bigotimes_{\left(U_{1}, \cdots, U_{n}\right)}^{(n)} \longrightarrow \bigotimes_{\left(V_{1}, \cdots, V_{n}\right)}^{(n)}
$$

depending only on the homotopy class of $\gamma$.

(3) These data are required to be compatible with the operadic compositions.

For any open $U \subset \mathbb{C}$ let $U_{\neq}^{n} \subset U^{n}$ be the set of $\left(z_{1}, \cdots, z_{n}\right)$ where $z_{i} \in U$ are such that $z_{i} \neq z_{j}$ for $i \neq j$. Given $n$ objects $V_{1}, \cdots, V_{n} \in \mathcal{V}$ and $\left(z_{1}, \cdots, z_{n}\right) \in D_{\neq}^{n}$, we denote

$$
\bigotimes_{\left(z_{1}, \cdots, z_{n}\right)} V_{i}=\bigotimes_{\left(U_{1}, \cdots, U_{n}\right)} V_{i},
$$

where the $U_{i}$ are sufficiently small open disks centered around $z_{i}$. This defines a local system on $D_{\neq}^{n}$ with values in $\mathcal{V}$. Since the embedding $D^{n} \neq \mathbb{C}_{\neq}^{n}$ is a homotopy equivalence, this local system extends canonically to a local system in $\mathbb{C}_{\neq}^{n}$, and so we can and will use the notation $\bigotimes_{\left(z_{1}, \cdots, z_{n}\right)} V_{i}$ for any $\left(z_{1}, \cdots, z_{n}\right) \in \mathbb{C}_{\neq}^{n}$.

We will refer to the above point of view on braided categories (many tensor products operations, labelled by $\left(U_{1}, \cdots, U_{n}\right)$ or $\left.\left(z_{1}, \cdots, z_{n}\right)\right)$ as the operadic point of view, as opposed to the classical point of view (one operation $\otimes$ together with braiding isomorphisms).

B. Braidings on derived categories and perverse sheaves. Let $\mathcal{V}$ be a braided abelian k-linear category, with $\otimes$ exact in each argument. We extend the braiding to $C^{b}(\mathcal{V})$, the category of bounded cochain complexes over $\mathcal{V}$ in a standard way. In other words, the operation $\otimes$ on $C(\mathcal{V})$ is defined to be the usual graded tensor product of comlexes (with differential defined by the Leibniz rule). The braiding on $C^{b}(\mathcal{V})$ is modified by the Koszul sign rule. That is, for objects $V, W \in \mathcal{V}$ and integers $m, n \in \mathbb{Z}$, the tensor product and braiding of the shifted objects are defined by

$$
(V[m]) \otimes(W[n])=(V \otimes W)[m+n], \quad R_{V[m], W[n]}=(-1)^{m n} R_{V, W}[m+n] .
$$

Because of bi-exactness of $\otimes$, the braided structure descends to $D^{b}(\mathcal{V})$, the bounded derived category of $\mathcal{V}$.

For any stratified complex manifold $(X, S)$ this induces a braided monoidal structure on the derived category $D^{b}(X, S, \mathcal{V})$ of $S$-constructible complexes on $X$ with values in $\mathcal{V}$. The abelian subcategory of perverse sheaves is typically not closed under $\otimes$. However, the exterior tensor product of perverse sheaves is perverse. 
That is, if $(X, S)$ and $(Y, T)$ are two stratified complex manifolds, we have the functor

$$
\bigotimes: \operatorname{Perv}(X, S, \mathcal{V}) \times \operatorname{Perv}(Y, T, \mathcal{V}) \longrightarrow \operatorname{Perv}(X \times Y, S \times T, \mathcal{V})
$$

Denoting by $\pi: X \times Y \rightarrow Y \times X$ the permutation, we have the external braiding isomorphism

$$
R_{\mathcal{F}, \mathcal{G}}: \mathcal{F} \otimes \mathcal{G} \longrightarrow \pi^{*}(\mathcal{G} \bowtie \mathcal{F}) .
$$

Note that a "locally constant perverse sheaf" on $X$ is in fact a local system placed in degree $(-\operatorname{dim} X)$. Therefore $R_{\mathcal{F}, \mathcal{G}}$ involves the Koszul sign rule even for "locally constant" $\mathcal{F}$ and $\mathcal{G}$.

Further, we extend the operadic point of view on the braiding to the external tensor products of perverse sheaves. That is, let $\left(X_{i}, S_{i}\right), i=1, \cdots, m$, be several stratified complex manifolds and $\mathcal{F}_{i} \in \operatorname{Perv}\left(X_{i}, S_{i}, \mathcal{V}\right)$. Then for any disjoint open disks $U_{1}, \cdots, U_{m}$ in the unit disk $D$ we have the object

$$
\bigotimes_{\left(U_{1}, \cdots, U_{m}\right)} \mathcal{F}_{i} \in \operatorname{Perv}\left(\prod X_{i}, \prod S_{i}, \mathcal{V}\right) .
$$

C. Factorizable perverse sheaves with values in a braided abelian category. Let now $\mathcal{V}$ be a braided monoidal abelian category, as before.

For any open set $U \subset \mathbb{C}$ we denote $\operatorname{Sym}(U)=\bigsqcup_{n \geqslant 0} \operatorname{Sym}^{n}(U)$. We denote by $S_{n, U}$ the diagonal stratification of $\operatorname{Sym}^{n}(U)$ and by $S_{U}$ the resulting stratification of $\operatorname{Sym}(U)$. In the case $U=\mathbb{C}$ we abbreviate $S_{n, U}$ to $S_{n}$ and $S_{U}$ to $S$. Denote by $\operatorname{Perv}\left(\operatorname{Sym}(U), S_{U} ; \mathcal{V}\right)$ the product of the categories $\operatorname{Perv}\left(\operatorname{Sym}^{n}(U), S_{n, U} ; \mathcal{V}\right)$. Thus an object of $\operatorname{Perv}\left(\operatorname{Sym}(U), S_{U} ; \mathcal{V}\right)$ is a collection $\mathcal{F}=\left(\mathcal{F}_{n}\right)_{n \geqslant 0}$ of perverse sheaves $\mathcal{F} \in \operatorname{Perv}\left(\operatorname{Sym}^{n}(U), S_{n, U} ; \mathcal{V}\right)$.

Let $\left(U_{1}, \cdots U_{m}\right)$ be a point of $E_{2}(m)$, i.e., a tuple of disjoint round open disks in $D$. Consider the Cartesian product $\prod_{i=1}^{m} \operatorname{Sym}\left(U_{i}\right)$ with its stratification $\Pi S_{U_{i}}$ formed by the products of the strata of the $S_{U_{i}}$. Since $U_{1}, \cdots, U_{m}$ are disjoint, we have a canonical identification ("addition map")

$$
a: \prod_{i=1}^{m} \operatorname{Sym}\left(U_{i}\right) \stackrel{\sim}{\longrightarrow} \operatorname{Sym}\left(\bigcup_{i=1}^{m} U_{i}\right) .
$$

Definition 3.2.5. A factorizable perverse sheaf on $\operatorname{Sym}(\mathbb{C})$ with values in $\mathcal{V}$ is an object $\mathcal{F}=\left(\mathcal{F}_{n}\right)$ of $\operatorname{Perv}(\operatorname{Sym}(\mathbb{C}), S ; \mathcal{V})$ together with the data of

$\left.{ }^{*}\right)$ For each $m \geqslant 0$ and each $\left(U_{1}, \cdots U_{m}\right) \in E_{2}(m)$, an isomorphism

$$
\mu_{U_{1}, \cdots, U_{m}}:\left.\mathrm{X}_{\left(U_{1}, \cdots, U_{m}\right)} \mathcal{F}\right|_{\operatorname{Sym}\left(U_{i}\right)} \longrightarrow a^{*}\left(\left.\mathcal{F}\right|_{\operatorname{Sym}\left(\bigcup U_{i}\right)}\right),
$$

these isomorphisms compatible with the operadic compositions. 
Examples 3.2.6. The condition "compatible with operadic compositions" includes, in particular, the following:

(a) Associativity for binary tensor products. That is, suppose we have three disjoint round disks $U_{1}, U_{2}, U_{3} \subset D$. Then the two ways of decomposing the map

$$
\mu_{U_{1}, U_{2}, U_{3}}:\left.\left.\bigotimes_{\left(U_{1}, U_{2}, U_{3}\right)} \mathcal{F}\right|_{\operatorname{Sym}\left(U_{i}\right)} \longrightarrow a^{*} \mathcal{F}\right|_{\operatorname{Sym}\left(U_{1} \cup U_{2} \cup U_{3}\right)}
$$

as a composition of two 2-variable $\mu$ 's, are equal.

(b) Compatibility with restrictions. Suppose $U_{1}, \cdots, U_{n}$ are disjoint disks as above and

$U_{i}^{\prime} \subset U_{i}$ are smaller disks. Then the restriction of $\mu_{U_{1}, \cdots, U_{n}}$ to $\left.\bigotimes_{\left(U_{1}^{\prime}, \cdots, U_{n}^{\prime}\right)} \mathcal{F}\right|_{\operatorname{Sym}_{\left(U_{i}\right)}}$ is equal to $\mu_{U_{1}^{\prime}, \cdots, U_{n}^{\prime}}$

We denote by $\operatorname{FPS}(\mathcal{V})$ the category formed by factorizable perverse sheaves on $(\operatorname{Sym}(\mathbb{C}), S)$ with values in $\mathcal{V}$.

Remark 3.2.7. Note that the isomorphism (3.2.4) holds for arbitrary, not necessarily round, disjoint topological disks $U_{1}, \cdots, U_{m} \subset \mathbb{C}$. One can show that for a factorizable perverse sheaf $\mathcal{F}$ and any such disjoint $U_{i}$ there is a natural identification of $a^{*}\left(\left.\mathcal{F}\right|_{\operatorname{Sym}\left(\cup U_{i}\right)}\right)$ with "the" tensor product of the $\left.\mathcal{F}\right|_{\operatorname{Sym}\left(U_{i}\right)}$. To formulate it canonically, we need a version of braided tensor product labelled by arbitrary sequence of disjoint topological disks. This can be achieved by forming an extended, colored version of the $E_{2}$-operad where each topological disk $U \subset \mathbb{C}$ is a separte color, cf. [13] §3.1.2. Since this generalization is not necessary for us, we do not pursue it here.

\subsection{From bialgebras to factorizable sheaves: the localization theo- rem}

A. Statement of results. Let $\mathrm{Br}_{n}$ be the Artin braid group on $n$ strands. Let

$$
j_{n}: \operatorname{Sym}_{\neq}^{n}(\mathbb{C})=X_{1^{n}}^{\mathbb{C}} \hookrightarrow \operatorname{Sym}^{n}(\mathbb{C})
$$

be the embedding of the open stratum (polynomials with distinct roots). Its fundamental group is $\mathrm{Br}_{n}$.

As before, let $\mathcal{V}$ be a $\mathbf{k}$-linear braided monoidal abelian category with $\otimes$ bi-exact. Recall that we have extended the braided monoidal structure to the bounded derived category $D^{b}(\mathcal{V})$.

For any object $V \in D^{b}(\mathcal{V})$ the tensor power $V^{\otimes n}$ a representation of $\mathrm{Br}_{n}$ and so gives a $D^{b}(\mathcal{V})$-valued local system on $\operatorname{Sym}_{\neq}^{n}(\mathbb{C})$ which we denote $\mathcal{L}_{n}(V)$. In the language of Proposition 3.2.1, various determinations of $V^{\otimes n}$ form, by the very definition, a $D^{b}(\mathcal{V})$-valued local system on the space $E_{2}(n)$ which is homotopy equivalent to $\operatorname{Sym}_{\neq}^{n}(\mathbb{C})$. This is $\mathcal{L}_{n}(V)$. We will view $\mathcal{L}_{n}(V)$ as a complex of locally constant sheaves on $\operatorname{Sym}_{\neq}^{n}(\mathbb{C})$. Recall also the notation $K_{n}=\operatorname{Sym}^{n}(\mathbb{R})$ for the real skeleton of $\operatorname{Sym}^{n}(\mathbb{C})$.

Let $\operatorname{PB}(\mathcal{V})$ be the category of primitive bialgebras in $\mathcal{V}$. 
Theorem 3.3.1. There is an equivalence of categories (called the localization functor)

$$
L: \operatorname{PB}(\mathcal{V}) \longrightarrow \operatorname{FPS}(\mathcal{V}), \quad A \mapsto L(A)=\left(L_{n}(A) \in \operatorname{Perv}\left(\operatorname{Sym}^{n}(\mathbb{C}), S_{n} ; \mathcal{V}\right)\right)
$$

with the following properties:

(a) The restriction $\left.L_{n}(\mathcal{F})\right|_{\operatorname{Sym}_{\neq}^{n}(\mathbb{C})}$ is identified with the local system $\mathcal{L}_{n}\left(A_{1}[1]\right)$ of determinations of $\left(A_{1}[1]\right)^{\otimes n}$.

(b) The total space of vanishing cycles $\Phi_{\text {tot }}\left(L_{n}(A)\right)$, see (2.3.5), is identified with $A_{n}$.

(c) More generally, the cubical diagram associated to the constructible sheaf $R_{K_{n}}\left(L_{n}(A)\right)$ is identified with $\mathbb{B}_{n}^{\dagger}(A)$, the nth cobar-cube of $A$.

(d) The stalk if $L_{n}(A)$ at 0 is identified with the $n$th bar-complex of $A$

$$
B_{n}^{\bullet}(A)=\left\{A_{1}^{\otimes n} \rightarrow \bigoplus_{l(\alpha)=n-1} \bigotimes_{i=1}^{n-1} A_{\alpha_{i}} \rightarrow \cdots \rightarrow \bigoplus_{\alpha_{1}+\alpha_{2}=n} A_{\alpha_{1}} \otimes A_{\alpha_{2}} \rightarrow A_{n}\right\}
$$

with grading normalized so that $A_{1}^{\otimes n}$ is in degree $(-n)$.

(e) Suppose $\mathcal{V}$ is rigid. Then for the primitive bialgebra $A^{*}$ dual to $A$ we have that $L_{n}\left(A^{*}\right)=\mathbb{D}\left(L_{n}(A)\right)$ is the Verdier dual to $L_{n}(A)$.

Remark 3.3.2. In part (a) of the theorem, $\left(A_{1}[1]\right)^{\otimes n}$ is identified with $A_{1}^{\otimes n}[n]$, that is, the $n$th tensor power of $A_{1}$ put in degree $(-n)$. However, the braid group action on $\left(A_{1}[1]\right)^{\otimes n}$ differs from that on $A_{1}^{\otimes n}$ by twisting with the sign character $\operatorname{Br}_{n} \rightarrow \Sigma_{n} \stackrel{\text { sgn }}{\rightarrow}\{ \pm 1\}$. This is because we extend the braided structure on $\mathcal{V}$ to the derived category of $\mathcal{V}$ by using the Koszul sign rule (3.2.3).

We denote $\operatorname{Sym}_{\neq}(\mathbb{C})=\bigsqcup_{n} \operatorname{Sym}_{\neq}^{n}(\mathbb{C})$ and write $j: \operatorname{Sym}_{\neq}(\mathbb{C}) \hookrightarrow \operatorname{Sym}(\mathbb{C})$ for the embedding. For an object $V \in \mathcal{V}$ we denote $\mathcal{L}(V[1])$ the local system on $\operatorname{Sym}_{\neq}(\mathbb{C})$ which on $\operatorname{Sym}_{\neq}^{n}(\mathbb{C})$ is $\mathcal{L}_{n}(V[1])$.

Theorem 3.3.3. Let $V$ be any object of $\mathcal{V}$. Then:

(a) We have $L\left(T_{!}(V)\right) \simeq j_{!} \mathcal{L}(V[1])$.

(b) We also have $L\left(T_{*}(V)\right) \simeq j_{*} \mathcal{L}(V[1])$

(c) Further, the canonical map $\alpha: T_{!}(V) \rightarrow T_{*}(V)$ is sent by $L$ to the canonical map $c:$ $j_{!} \rightarrow j_{*}$, and the Nichols algebra $T_{! *}(V)$ corresponds, under $L$, to the intersection cohomology extension $j_{! *} \mathcal{L}(V[1])$.

Theorems 3.3.1 and 3.3.3 will be proved in the next Ch. 4. Here we note the following corollary which was proved, in an equivalent form, in [15], Th. 1.3.

Corollary 3.3.4. We have an identification

$$
H^{j}\left(\operatorname{Br}_{n}, V^{\otimes n} \otimes_{\mathbf{k}} \operatorname{sgn}\right) \simeq \operatorname{Tor}_{n-j, n}^{T_{*}(V)}(\mathbf{1}, \mathbf{1}) .
$$


Proof of the corollary: Let $j_{n}: \operatorname{Sym}_{\neq}^{n}(\mathbb{C}) \rightarrow \operatorname{Sym}^{n}(\mathbb{C})$ be the embedding. By 3.3.3 (b) $L_{n}\left(T_{*}(V)\right) \simeq j_{n *} \mathcal{L}_{n}(V[1])$. Because of the quasi-homogeneity of the diagonal stratification $S$ of $\operatorname{Sym}^{n}(\mathbb{C})$ (the $\mathbb{C}^{*}$-action coming from the standard dilation action on $\mathbb{C}$ ), the stalk of $j_{n *} \mathcal{L}_{n}(V[1])$ at 0 is identified with the complex

$$
R \Gamma\left(\operatorname{Sym}^{n}(\mathbb{C}), j_{n *} \mathcal{L}_{n}(V[1])\right) \simeq R \Gamma\left(\operatorname{Sym}_{\neq}^{n}(\mathbb{C}), \mathcal{L}_{n}(V[1])\right)
$$

and the complex in the RHS calculates $H^{\bullet}\left(\mathrm{Br}_{n}, V^{\otimes n} \otimes_{\mathbf{k}} \operatorname{sgn}\right)[n]$. So our statement follows from Theorem 3.3.1(d).

B. Example: Yetter-Drinfeld modules and Hurwitz spaces. Let us explain the relation of our results with the work [15] of Ellenberg, Tran and Westerland.

Let $G$ be a finite group with multiplication $m: G \times G \rightarrow G$. A Yetter-Drinfeld $G$-module (YD $G$-module, for short) is a finite-dimensional $G$-graded k-vector space $V=\bigoplus_{g \in G} V_{g}$ with a right $G$-action such that $V_{g} \cdot h=V_{h^{-1} g h}$; this is the same as a module over the Drinfeld double of the group ring $\mathbf{k}[G]$. Such objects form a $\mathbf{k}$-linear abelian rigid braided monoidal category $\mathcal{Y}_{G}$ with $\otimes$ being the usual graded tensor product and braiding given by

$$
R(v \otimes w)=w \otimes(v \cdot h), \quad v \in V_{g}, w \in V_{h} .
$$

Alternatively, we can consider character sheaves, i.e., conjugation equivariant sheaves $F$ of finite-dimensional k-vector spaces on $G$ as a discrete space. Such a sheaf $F$ gives a YD $G$-module $V=\Gamma(G, F)$ of global sections, and the monoidal operation corresponds to the convolution of sheaves $F * F^{\prime}=m_{*}\left(F \otimes F^{\prime}\right)$. The braided category of character sheaves (i.e., the category $\mathcal{Y}_{G}$ ) can be, therefore, seen as a categorification of the commutative ring of class functions on $G$ under convolution. See [2] §3.2, [44] for more background.

If $C \subset G$ is a conjugation invariant subset, then the constant sheaf $\underline{\mathbf{k}}_{C}$ is a character sheaf with corresponding YD $G$-module $V=\mathbf{k} C$ (the $\mathbf{k}$-span of $C$ ). In this case the $\mathrm{Br}_{n}$-module $V^{\otimes n}=\mathrm{k} C^{n}$ is the permutation module corresponding to the $\mathrm{Br}_{n}$-action on the Cartesian power $C^{n}$ given on the standard generators by

$$
\sigma_{i}\left(g_{1}, \cdots, g_{n}\right)=\left(g_{1}, \cdots, g_{i-1}, g_{i+1}, g_{i+1}^{-1} g_{i} g_{i+1}, g_{i+2}, \cdots, g_{n}\right), \quad i=1, \cdots, n-1 .
$$

(This action reflects the embedding of $\mathrm{Br}_{n}$ into the automorphism group of the free group.) The Hurwitz space associated to $G, C$ and $n$ is the unramified covering

$$
p_{n}: \operatorname{Hur}_{G, n}^{C} \longrightarrow \operatorname{Sym}_{\neq}^{n}(\mathbb{C})
$$

associated to the $\mathrm{Br}_{n}$-set $C^{n}$. It has the following relations to moduli of branched covers (see [16] §2 for a systematic discussion):

(1) Let $D=\{|z|<1\}$ be the unit disk in $\mathbb{C}$ and $\bar{D}$ be its closure $|z| \leqslant 1$. The complex manifold

$$
\operatorname{Hur}_{G, n}^{C}(D)=p_{n}^{-1}\left(\operatorname{Sym}_{\neq}^{n}(D)\right)
$$

parametrizes $G$-coverings $\pi: Y \rightarrow \bar{D}$ ramified at some $n$ points in $D$, with monodromies around these points liying in $C$, together with a distinguished point $y \in \pi^{-1}(1)$. 
(2) The quotient Deligne-Mumford stack $\operatorname{Hur}_{G, n}^{C} / / G$ parametrizes $G$-coverings of the affine line $\mathbb{A}^{1}$ ramified at $n$ points with monodromies around these points lying in $C$.

Note that the intrepretation (1) gives a map $M: \operatorname{Hur}_{G, n}^{C}(D) \rightarrow G$ given by the counterclockwise monodromy around the boundary of $\bar{D}$, and so we have a disjoint union decomposition (G-grading)

$$
\operatorname{Hur}_{G, n}^{C}(D)=\bigsqcup_{g \in G} \operatorname{Hur}_{G, n}^{C}(D)_{g}, \quad \operatorname{Hur}_{G, n}^{C}(D)_{g}:=M^{-1}(g) .
$$

Together with the natural $G$-action on $\operatorname{Hur}_{G, n}^{C}(D)$ (change of $y$ ) this makes $\operatorname{Hur}_{G, n}^{C}(D)$ into a "YD $G$-module in the category of complex manifolds".

Thus the (untwisted) local system $\mathcal{L}_{n}(V)$ of determinations of $V^{\otimes n}$ is the direct image

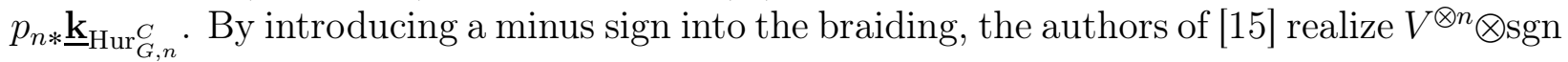
as $p_{n * \underline{\mathbf{k}}_{\mathrm{Hur}_{G, n}^{C}}}$, which, by Corollary 3.3 .4 (i.e., Thm. 1.3 of [15]) identifies the topological cohomology of Hurwitz spaces as

$$
H^{j}\left(\operatorname{Hur}_{G, n}^{C}, \mathbf{k}\right) \simeq \operatorname{Tor}_{n-j, n}^{T_{*}(V)}(\mathbf{k}, \mathbf{k}) .
$$

We note that Theorem 3.3.3(c) provides a similar geometric intnerpretation of the Nichols algebra $T_{! *}(V)$ :

Corollary 3.3.5. We have an identification

$$
\operatorname{Tor}_{n-j, n}^{T_{! *}(V)}(\mathbf{k}, \mathbf{k})=\mathbb{H}^{j}\left(\operatorname{Sym}^{n}(\mathbb{C}), j_{! *} p_{n *} \mathbf{k}_{\mathrm{Hur}_{G, n}^{C}}\right) .
$$

It is interesting whether the RHS of this identification, involving the intersection cohomology extension of a local system, can be described in terms of intersection cohomology of some natural compactification of the Hurwitz space.

Remark 3.3.6. The formulation of [15] proceeds in the dual form, using Ext-cohomology, not Tor-homology of $T_{*}(V)$, which is related to the homology rather than cohomology of $\operatorname{Hur}_{G, n}^{C}$. This has the convenience that $\operatorname{Ext}^{\bullet}(\mathbf{k}, \mathbf{k})$ forms an algebra, rather than a coalgebra. The fact that this algebra is (graded) braided-commutative reflects the phenomenon, mentioned in the Introduction, that the Koszul dual of a Hopf algebra is an $E_{2}$-algebra. In fact, the appearance of $E_{2}$-algebras in this context is more fundamental, since:

(1) The Hurwitz spaces $\operatorname{Hur}_{G, n}^{C}(D)$ themselves, taken for all $n \geqslant 0$, form, naturally, an $E_{2}$-algebra in the (braided monoidal) category of YD-modules in complex manifolds.

(2) Similarly, the quotients $\operatorname{Hur}_{G, n}^{C}(D) / / G$ form an $E_{2}$-algebra in the more usual (symmetric monoidal) category of analytic stacks.

These algebra structures are given by appropriate versions of the gluing maps of [16] §2.3. 


\section{Proof of Localization Theorem: Cousin complexes}

\subsection{The Cousin sheaves of a coalgebra}

Our proof of Theorem 3.3.1 consists in constructing the perverse sheaf $L_{n}(A)$ as an explicit complex of sheaves on $\operatorname{Sym}^{n}(\mathbb{C})$, which we call the Cousin complex. In this section we describe the sheaves constituting this complex.

A. Objects $A_{\mathbf{z}}$ and local systems $\mathcal{L}_{\alpha}(A)$. Let $A=\bigoplus_{n=0}^{\infty}$ be a graded object in $\mathcal{V}$ with $A_{0}=\mathbf{1}$. Let $\mathbf{z} \in \operatorname{Sym}^{n}(\mathbb{C})$ be a point understood as an unordered divisor $\mathbf{z}=\sum_{i=1}^{d} \lambda_{i} z_{i}$ with $\lambda_{i} \geqslant 1$ and $z_{i} \neq z_{j}$. The operadic point of view on the braided structure in $\mathcal{V}$ allows us to form the object

$$
A_{\mathbf{z}}=\bigotimes_{\left(z_{1}, \cdots, z_{d}\right)} A_{\lambda_{i}}
$$

which is canonically (up to a unique isomorphism) independent on the ordering of $z_{1}, \cdots, z_{d}$. Given an unordered partition $\lambda=\left(\lambda_{1}, \cdots, \lambda_{d}\right) \in \mathcal{P}(n)$, the objects $A_{\mathbf{z}}, \mathbf{z} \in X_{\lambda}^{\mathbb{C}}$, form a local system on the complex stratum $X_{\lambda}^{\mathbb{C}}$. We denote this local system $\mathcal{L}_{\lambda}(A)$. For $\lambda=1^{n}$ we get the local system $\mathcal{L}_{n}\left(A_{1}\right)$ of determinations of $A_{1}^{\otimes n}$ on $\operatorname{Sym}_{\neq}^{n}(A)$, see $\S 3.2 \mathrm{~A}$.

B. Cousin sheaves $\mathcal{E}^{\alpha}(A)$ of a coalgebra. Let us further assume that $A$ is a graded coassociative coalgebra in $\mathcal{V}$ with counit given by the projection to $A_{0}=\mathbf{1}$, satisfying the primitivity condition (2) of Definition 2.4.10.

Since the objects $A_{\mathbf{z}}$ are canonically determined by the points $\mathbf{z} \in \operatorname{Sym}^{n}(\mathbb{C})$, it is natural to consider the following.

Problem 4.1.1. Arrange the objects $A_{\mathbf{z}}$ into a constructible sheaf on $\operatorname{Sym}^{n}(\mathbb{C})$ so that these objects are the stalks and the sheaf structure (generalization maps) is given by the comultiplication $\Delta$ in $A$.

However, this is impossible unless $\Delta$ is cocommutative.

Example 4.1.2. Let $n=2$. After quotienting by translations, $\operatorname{Sym}^{2}(\mathbb{C})$ becomes the complex plane, with the open stratum, the reduction of $X_{1^{2}}^{\mathbb{C}}=\operatorname{Sym}_{\neq}^{2}(\mathbb{C})$ being $\mathbb{C}^{*}$ and the closed stratum, the reduction of $X_{(2)}^{\mathbb{C}}$, being the point 0 . So the local system $\mathcal{L}_{1^{2}}(A)$ on $\mathbb{C}^{*}$ has monodromy $R: A_{1} \otimes A_{1} \rightarrow A_{1} \otimes A_{1}$, and the stalk of $\mathcal{L}_{(2)}(A)$ at 0 is $A_{2}$. To combine them into a constructible sheaf on $\mathbb{C}$, we need to specify a morphism $\varepsilon: A_{2} \rightarrow A_{1} \otimes A_{1}$ such that $R \circ \varepsilon=\varepsilon$, so the comultiplication cannot do this in general.

Nevertheless we have interesting partial solutions to Problem 4.1.1, which produce sheaves not on all $\operatorname{Sym}^{n}(\mathbb{C})$ but on some subspaces.

Proposition 4.1.3. Let $\alpha \in \mathbf{2}^{n-1}$. The comultiplication $\Delta$ in $A$ defines a sheaf $\widetilde{\mathcal{E}}^{\alpha}(A)$ on $X_{\alpha}^{\mathfrak{I}}$ whose stalk at any $\mathbf{z} \in X_{\alpha}^{\mathfrak{I}}$ is identified with $A_{\mathbf{z}}$ and the generalization maps are given by the appropriate components of $\Delta$. 
Remark 4.1.4. The reason why it becomes possible to arrange the $A_{\mathbf{z}}$ into a sheaf on $X_{\alpha}^{\mathfrak{I}}$ is that the intersection of each $X_{\alpha}^{\mathfrak{I}}$ with each $X_{\lambda}^{\mathbb{C}}$ is contractible, see Proposition 2.2.5.

Proof of the proposition: Recall the "real parts" projection

$$
\rho_{\alpha}: X_{\alpha}^{\mathfrak{I}} \longrightarrow \prod_{\nu=1}^{k} \operatorname{Sym}^{\alpha_{\nu}}(\mathbb{R})
$$

For each $m \geqslant 0$ let $\mathbb{B}_{m}^{\dagger}(A)$ be the $m$ th cobar-cube associated to the coalgebra structure on $A$ and let $R_{m}(A)$ be the constructible sheaf on the stratified space $\left(K_{m}=\operatorname{Sym}^{m}(\mathbb{R}), K_{m, S}\right)$ corresponding to $\mathbb{B}_{m}^{\dagger}(A)$. Define, using the "classical" point of view (one tensor product operation) on the braided structure:

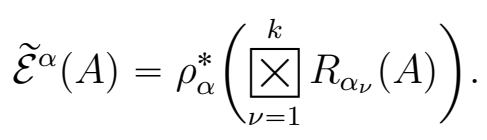

Let us show why, at the level of stalks, this gives the desired answer.

Let first $m \geqslant 0$ and consider a point $\mathbf{x}$ of $\operatorname{Sym}^{m}(\mathbb{R})$ written as an ordered divisor $m_{1} x_{1}+$ $\cdots+m_{p} x_{p}$, with $x_{1}<\cdots x_{p}$ and $m_{1}+\cdots+m_{p}=m$. The stalk of $R_{m}(A)$ at $\mathbf{x}$ is, in the "classical" notation

$$
R_{m}(A)_{\mathbf{x}}=A_{m_{1}} \otimes \cdots A_{m_{p}} .
$$

Let now $\alpha=\left(\alpha_{1}, \cdots, \alpha_{k}\right) \in \mathbf{2}^{n-1}$ and $\mathbf{z}=\left\{z_{1}, \cdots, z_{n}\right\} \in X_{\alpha}^{\mathfrak{I}}$. In other words, according to the increasing order of the $\operatorname{Im}\left(z_{j}\right)$, the $z_{j}$ are subvidided into $k$ groups, so that the elements the $\nu$ th group, of cardinality $\alpha_{\nu}$, have the same imaginary part. The $\nu$ th component of the projection $\rho_{\alpha}$ is the map $X_{\alpha}^{\mathfrak{I}} \rightarrow \operatorname{Sym}^{\alpha_{\nu}}(\mathbb{R})$ which sends $\mathbf{z}$ into the points represented by the real parts of the $z_{j}$ from the $\nu$ th group. Let us now write $\mathbf{z}$ itself as a divisor $\sum_{i=1}^{d} \lambda_{i} z_{i}$ with $z_{1}, \cdots, z_{d}$ being distinct and $\lambda_{i}>0$. From the above we conclude that in the "classical" notation, the stalk of $\widetilde{\mathcal{E}}^{\alpha}(A)$ at $\mathbf{z}$ would be simply the tensor product $\bigotimes_{i=1}^{d} A_{\lambda_{i}}$, taken in the lexicographic order with respect first, to the imaginary and then to the real parts of $z_{1}, \cdots, z_{d}$. This is precisely $A_{\mathbf{z}}$.

Let us form the complexes (recall that $j_{\alpha *}$ means the derived direct image)

$$
\mathcal{E}^{\alpha}(A)=j_{\alpha *} \widetilde{\mathcal{E}}^{\alpha}(A), \quad j_{\alpha}: X_{\alpha}^{\mathfrak{I}} \hookrightarrow \operatorname{Sym}^{n}(\mathbb{C}) .
$$

We call $\mathcal{E}^{\alpha}(A)$ the $\alpha$ th Cousin sheaf of $A$; it will be proved later that is indeed quasiisomorphic to a single sheaf in degree 0 . In order to establish this and other properties of $\mathcal{E}^{\alpha}(A)$ we need some preparations.

C. Contingency matrices and double cosets. Let $\xi=\left(\xi_{1}, \cdots, \xi_{r}\right)$ and $\eta=\left(\eta_{1}, \cdots, \eta_{s}\right)$ be two ordered partitions of the same number $m=\sum \xi_{i}=\sum \eta_{j}$. By a $(\xi, \eta)$-contingency 
matrix $^{1}$ we will mean an $r \times s$ matrix $\zeta=\left\|\zeta_{i j}\right\|_{i=1, \cdots, r}^{j=1, \cdots, s}$ with entries in $\mathbb{Z}_{\geqslant 0}$ such that

$$
\sum_{j} \zeta_{i j}=\xi_{i}, \quad \forall i=1, \cdots, r, \quad \sum_{i} \zeta_{i j}=\eta_{j}, \quad \forall j=1, \cdots, s,
$$

and, in addition, each row and each column has a nonzero entry. We denote the set of $(\xi, \eta)$-contingency matrices by $S(\xi, \eta)$.

Example 4.1.8. Let $\eta=1^{m}$ be the maximal ordered partition. Then a $\left(\xi, 1^{m}\right)$-contingency matrix is the same as a shuffle of type $\xi$, i.e., a sequence $\left(X_{1}, \cdots, X_{r}\right)$ of subsets of $\{1, \cdots, m\}$ with $\left|X_{i}\right|=\xi_{i}$, forming a disjoint decomposition. In particular,

$$
\left|S\left(\xi, 1^{m}\right)\right|=\frac{m !}{\left(\xi_{1}\right) ! \cdots\left(\xi_{r}\right) !} .
$$

Let $F$ be a field and Let $P(\xi) \subset G L_{m}(F)$ be the parabolic subgroup of block upper triangular matrices with blocks of sizes $\xi_{1}, \cdots, \xi_{r}$, and similarly for $P(\eta)$. Recall that $\Sigma_{m}$ denotes the symmetric group, i.e., the Weyl group of $G L_{m}(F)$. Let $\Sigma(\xi)=\Sigma_{\xi_{1}} \times \cdots \times \Sigma_{\xi_{r}} \subset$ $\Sigma_{m}$ be the Weyl group of the Levi subgroup of $P(\xi)$, and similarly for $\Sigma(\eta)$.

Proposition 4.1.9. We have the identifications (parabolic Bruhat decomposition)

$$
P(\xi) \backslash G L_{m}(F) / P(\eta) \simeq S(\xi, \eta) \simeq \Sigma(\xi) \backslash \Sigma_{m} / \Sigma(\eta) .
$$

Proof: This is well known. For a discussion of the first identification (possible relative positions of a pair of partial flags in $F^{m}$ ) see, e.g., [8]. As for the second identification, see [14] Lemma 3.3, which has been visualized as the "balls in boxes" picture in [50] $§ 6$.

Given $\zeta \in S(\xi, \eta)$, we have an ordered partition $\operatorname{lex}(\zeta)$ of $m$ obtained by reading the nonzero entries of $\zeta$ in the lexicographic order, starting from the first column $\zeta_{1,1}, \cdots, \zeta_{s, 1}$, then the second column $\zeta_{1,2}, \cdots, \zeta_{s, 2}$ and so on. Note that $\operatorname{lex}(\zeta)>\eta$ is a refinement of $\eta$ (but not necessrily a refinement of $\xi$ ).

Let now $n \geqslant 0$ and $\alpha, \beta, \gamma \in \mathbf{2}^{n-1}$ be three ordered partitions of $n$ such that $\alpha \geqslant \beta \leqslant \gamma$. Writing $\beta=\left(\beta_{1}, \cdots, \beta_{k}\right)$, the inequality $\beta \leqslant \gamma$ means that each $\beta_{i}$ is the sum of a string of consecutive $\gamma$ 's:

$$
\beta_{i}=\gamma_{r_{i}}+\gamma_{r_{i}+1}+\cdots+\gamma_{r_{i+1}-1}, \quad \text { for some } 1=r_{1}<\cdots<r_{k+1}=l(\gamma)+1
$$

Similarly, the inequality $\alpha \geqslant \beta$ means that

$$
\beta_{i}=\alpha_{s_{i}}+\alpha_{s_{i}+1}+\cdots+\alpha_{s_{i+1}-1}, \quad \text { for some } \quad 1=s_{1}<\cdots<s_{k+1}=l(\alpha)+1 .
$$

\footnotetext{
${ }^{1}$ the statisticians call such matrices contingency tables with margins $(\xi, \eta)$ (the name given by Karl Pearson at the beginning of the last century), cf. [14].
} 
This means that for each $i$ we have two ordered partitions of $\beta_{i}$ which we denote

$$
\alpha / \beta_{i}=\left(\alpha_{s_{i}}, \alpha_{s_{i}+1}, \cdots, \alpha_{s_{i+1}-1}\right), \quad \gamma / \beta_{i}=\left(\gamma_{r_{i}}, \gamma_{r_{i}+1}, \cdots, \gamma_{r_{i+1}-1}\right)
$$

We put

$$
S(\alpha, \beta, \gamma)=\prod_{i=1}^{k} S\left(\alpha / \beta_{i}, \gamma / \beta_{i}\right)
$$

Thus, an element of $S(\alpha, \beta, \gamma)$ is a sequence $\vec{\zeta}=\left(\zeta^{(1)}, \cdots, \zeta^{(k)}\right)$ of contingency matrices $\zeta^{(i)} \in S\left(\alpha / \beta_{i}, \gamma / \beta_{i}\right)$. For such a sequence $\vec{\zeta}$ we denote by $\operatorname{Lex}(\vec{\zeta})$ the ordered partition of $n$ formed by writing $\operatorname{lex}\left(\zeta^{(1)}\right), \cdots, \operatorname{lex}\left(\zeta^{(k)}\right)$ in a single sequence. We note that $\operatorname{Lex}(\vec{\zeta}) \geqslant \beta$ is a refinement of $\beta$ (but not necessarily a refimenent of $\alpha$ ).

D. Properties of the Cousin sheaves. Recall that $A=\oplus_{n} A_{n}$ is a graded coalgebra in the braided category $\mathcal{V}$, as in $\S \mathrm{B}$. We study the complexes $\mathcal{E}^{\alpha}(A)$ defined in (4.1.7).

For any ordered partition $\theta=\left(\theta_{1}, \cdots, \theta_{p}\right) \in 2^{n-1}$ we denote $A_{\theta}=A_{\theta_{1}} \otimes \cdots \otimes A_{\theta_{p}}$.

For two ordered partitions $\xi, \eta \in \mathbf{2}^{m-1}$ and a contingency matrix $\zeta=\left\|\zeta_{i j}\right\| \in S(\xi, \eta)$ we denote $A_{\zeta}=\bigotimes_{i, j} A_{\zeta_{i j}}$, the tensor product in the sense of (3.2.2), formed with respect to positioning $A_{\zeta_{i j}}$ at the point $(i, j) \in \mathbb{R}^{2}=\mathbb{C}$, i.e., at the complex number $i+j \sqrt{-1}$.

For $\alpha \geqslant \beta=\left(\beta_{1}, \cdots, \beta_{k}\right) \leqslant \gamma \in 2^{n-1}$ and a sequence of matrices $\vec{\zeta}=\left(\zeta^{(1)}, \cdots, \zeta^{(k)}\right) \in$ $S(\alpha, \beta, \gamma)$ we put $A_{\vec{\zeta}}=A_{\zeta^{(1)}} \otimes \cdots \otimes A_{\zeta^{(k)}}$.

Proposition 4.1.12. (a) The restriction of $\mathcal{E}^{\alpha}(A)$ to any fine cell $X_{[\beta: \gamma]}, \beta \leqslant \gamma$, vanishes unless $\alpha \geqslant \beta$.

(b) Suppose $\alpha \geqslant \beta \leqslant \gamma$. Then the stalk of $\mathcal{E}^{\alpha}(A)$ at any point $\mathbf{z} \in X_{[\beta: \gamma]}$ is quasiisomorphic to the object

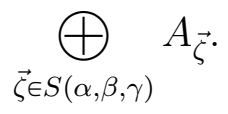

in degree 0.

This implies immediately:

Corollary 4.1.13. $\mathcal{E}^{\alpha}(A)$ is quasi-isomorphic to a single sheaf in degree 0 which is constructible with respect to the stratification by fine cells.

To prove Proposition 4.1.12, let $\mathbf{z} \in X_{[\beta: \gamma]}$ and $U \subset \operatorname{Sym}^{n}(\mathbb{C})$ be a small ball around $\mathbf{z}$. The stalk of $\mathcal{E}^{\alpha}(A)$ at $\mathbf{z}$ is, by definition, $R \Gamma\left(U \cap X_{\alpha}^{\mathfrak{I}}, \widetilde{\mathcal{E}}^{\alpha}(A)\right)$. So it is enough to show the following.

Proposition 4.1.14. (1) Unless $\alpha \geqslant \beta$, the intersection $U \cap X_{\alpha}^{\mathfrak{I}}$ is empty.

(2) Assume $\alpha \geqslant \beta$. Then: 
(2a) $U \cap X_{\alpha}^{\mathfrak{I}}$ is the union of contractible components $T_{\vec{\zeta}}$ labelled by $\vec{\zeta} \in S(\alpha, \beta, \gamma)$.

(2b) Further, consider the stratification of $X_{\alpha}^{\mathfrak{I}}$ given by $X_{\alpha}^{\mathfrak{I}}=\bigsqcup_{\varepsilon \geqslant \alpha} X_{[\alpha: \varepsilon]}$. The intersection of $T_{\vec{\zeta}}$ with each stratum $X_{[\alpha: \varepsilon]}$ is contractible or empty, and the minimal stratum meeting $T_{\vec{\zeta}}$ is $X_{[\alpha: \operatorname{Lex}(\vec{\zeta})]}$.

Proof: Part (1) is clear since the closure of $X_{\alpha}^{\mathfrak{I}}$ is the union of $X_{[\beta: \gamma]}$ for $\alpha \geqslant \beta \leqslant \gamma$.

Let us prove part (2). Since $\mathbf{z} \in X_{[\beta: \gamma]} \subset X_{\beta}^{\mathfrak{I}}$, we have a decomposition $\mathbf{z}=\mathbf{z}_{1}+\cdots+\mathbf{z}_{k}$ where each $\mathbf{z}_{i} \in \operatorname{Sym}^{\beta_{i}}(\mathbb{C})$ has the imaginary parts of all its component points equal; denoting this common value $\operatorname{Im}\left(\mathbf{z}_{i}\right)$, we further have $\operatorname{Im}\left(\mathbf{z}_{1}\right)<\cdots<\operatorname{Im}\left(\mathbf{z}_{k}\right)$. Further, the condition that $\mathbf{z} \in X_{[\beta: \gamma]}$ means that each $\mathbf{z}_{i}$, positioned on a horizontal line $\operatorname{Im}(z)=$ const, has multiplicities $\gamma_{r_{i}}, \gamma_{r_{i}+1}, \cdots, \gamma_{r_{i+1}-1}$, see (4.1.10), if read from left to right on that line.

Now suppose we deform $\mathbf{z}$ to a point $\mathbf{z}^{\prime}$ in a small ball $U$ around $\mathbf{z}$, such that $\mathbf{z}^{\prime} \in X_{\alpha}^{\mathfrak{I}}$. Recall that $\alpha \geqslant \beta$, in particular, $\beta_{i}$ is represented as the sum (4.1.11).

Such a deformation deforms each $\mathbf{z}_{i}$ to some $\mathbf{z}_{i}^{\prime}$. The deformation of $\mathbf{z}_{i}$ to $\mathbf{z}_{i}^{\prime}$ subdivides each $\mathbf{z}_{i}$ to a sum $\mathbf{z}_{i, s_{i}}+\mathbf{z}_{i, s_{i}+1}+\cdots+\mathbf{z}_{i, s_{i+1}-1}$ so that $\mathbf{z}_{i, \nu}$ moves to a divisor $\mathbf{z}_{i, \nu}^{\prime}$ elying on the $\nu$ th horizontal line $\operatorname{Im}(z)=$ const featured in the definition of the stratum $X_{\alpha}^{\mathfrak{I}}$. Such a separation defines a contingency matrix $\zeta^{(i)} \in S\left(\alpha / \beta_{i}, \gamma / \beta_{i}\right)$. This matrix is just the matrix of multiplicities of the divisor $\mathbf{z}_{i}^{\prime}$. So the tensor product $A_{\mathbf{z}_{i}^{\prime}}$ is identified with $A_{\zeta^{(i)}}$.

Now, deforming the whole of $\mathbf{z}$ to $\mathbf{z}^{\prime}$ amounts to deforming each $\mathbf{z}_{i}$ to a $\mathbf{z}_{i}^{\prime}$ as above, and this defines a sequence of contingency matrices $\vec{\zeta}=\left(\zeta^{(1)}, \cdots, \zeta^{(k)}\right)$. The component $T_{\vec{\zeta}}$ of the intersection $U \cap X_{\alpha}^{\mathfrak{I}}$ consists, by definition, of deformations $\mathbf{z}^{\prime}$ giving the sequence $\vec{\zeta}$. The space $\Gamma\left(T_{\vec{\zeta}}, \widetilde{\mathcal{E}}^{\alpha}(A)\right)$ is then identified with the tensor product $A_{\mathbf{z}^{\prime}}$ for any $\mathbf{z}^{\prime} \in T_{\vec{\zeta}}$, and this tensor product is identified, by the above, with $A_{\vec{\zeta}}$.

\subsection{The Cousin complexes of a bialgebra as perverse sheaves}

A. Definition of the Cousin complexes. Assume now that $A$ is a primitive bialgebra in $\mathcal{V}$. For $\alpha, \beta \in \mathbf{2}^{n-1}$ and $d>0$ we write $\alpha>_{d} \beta$ if $\alpha>\beta$ and $l(\alpha)=l(\beta)+d$.

Let $\alpha=\left(\alpha_{1}, \cdots, \alpha_{k+1}\right)>_{1} \beta=\left(\beta_{1}, \cdots, \beta_{k}\right)$. Define the morphism of sheaves on $X_{\beta}^{\mathfrak{I}}$

$$
\delta_{\alpha, \beta}^{\prime}: j_{\beta}^{*} \mathcal{E}^{\alpha}(A)=j_{\beta}^{*} j_{\alpha *} \widetilde{\mathcal{E}}^{\alpha}(A) \longrightarrow \widetilde{\mathcal{E}}^{\beta}(A)
$$

using the multiplication $\mu$ in $A$. More precisely, $\alpha>_{1} \beta$ means that $\beta$ is obtained from $\alpha$ by combining together two consecutive parts, say $\alpha_{i}$ and $\alpha_{i+1}$ :

$$
\beta=\left(\alpha_{1}, \cdots, \alpha_{i-1}, \alpha_{i}+\alpha_{i+1}, \alpha_{i+2}, \cdots, \alpha_{k+1}\right)
$$

which is a particuilar case of (4.1.11). Accordingly, we apply a particular case of the reasoning of Proposition 4.1.14(2a). That is, let $\mathbf{z}$ be a point of $X_{\beta}^{\mathfrak{I}}$, considered as an unordered divisor. The fact that $\mathbf{z} \in X_{\beta}^{\mathfrak{I}}$ means that $\mathbf{z}$ is decomposed into a sum $\mathbf{z}_{1}+\cdots+\mathbf{z}_{k}$, where $\mathbf{z}_{j}$ is of degree $\beta_{j}$ and its points have the same imaginary part. The stalk of $j_{\beta}^{*} j_{\alpha *} \widetilde{\mathcal{E}}^{\alpha}(\mathrm{A})$ at $\mathbf{z}$ is, 
by definition, the space of sections of $\widetilde{\mathcal{E}}_{\alpha}(A)$ on $U \cap X_{\alpha}^{\mathfrak{I}}$, where $U$ is a small ball around $\mathbf{z}$ in $\operatorname{Sym}^{n}(\mathbb{C})$. This intersection consists of several components, labelled by the ways of decomposing $\mathbf{z}_{i}$ into a sum of effective divisors

$$
\mathbf{z}_{i}^{1}+\mathbf{z}_{i}^{2}, \quad \mathbf{z}_{i}^{1} \in \operatorname{Sym}^{\alpha_{i}}(\mathbb{C}), \mathbf{z}_{i}^{2} \in \operatorname{Sym}^{\alpha_{i+1}}(\mathbb{C})
$$

The component, denote it $T=T\left(\mathbf{z}_{i}^{1}, \mathbf{z}_{i}^{2}\right)$, corresponding to such a decomposition, is obtaind by increasing the imaginary parts of all the points of $\mathbf{z}_{i}^{1}$ by the small increment $\varepsilon$, getting a new divisor $\mathbf{w}_{i}^{1}$ and similarly decreasing the imaginary parts of the points of $\mathbf{z}_{i}^{2}$ getting a divisor $\mathbf{w}_{i}^{2}$. The divisor

$$
\mathbf{w}=\mathbf{z}_{1}+\cdots+\mathbf{z}_{i-1}+\mathbf{w}_{i}^{1}+\mathbf{w}_{i}^{2}+\mathbf{z}_{i+1}+\cdots+\mathbf{z}_{k-1}
$$

represents a point in $T$ mapped by $\rho_{\alpha}: X_{\alpha}^{\mathfrak{I}} \rightarrow \prod \operatorname{Sym}^{\alpha_{i}}(\mathbb{R})$ into the stratum which is minimal possible for $\mathrm{T}$ and so the space of sections of the sheaf $\widetilde{\mathcal{E}}^{\alpha}(A)$ over $T$ is canonically identified with $A_{\mathrm{w}}$. The multiplication $\mu$ in $A$ gives a morphism $A_{\mathrm{w}} \rightarrow A_{\mathrm{z}}$. We define the action of $\delta_{\alpha, \beta}^{\prime}$ on the stalk of $j_{\beta}^{*} j_{\alpha *} \widetilde{\mathcal{E}}^{\alpha}(A)$ at $\mathbf{z}$ to be the map $A_{\mathbf{w}} \rightarrow A_{\mathbf{z}}$ given by multiplication in $A$.

Proposition 4.2.2. The maps of stalks defined above commute with the generalization maps and so define a morphism of sheaves $\delta_{\alpha, \beta}^{\prime}: j_{\beta}^{*} j_{\alpha *} \widetilde{\mathcal{E}}^{\alpha}(A) \longrightarrow \widetilde{\mathcal{E}}^{\beta}(A)$.

Proof: The generalization maps are given by the comultiplication in $A$, the maps of stalks we defined are given by the multiplication. The commutation of the two sets of maps follows from the compatibility of multiplication and comultiplication in $A$.

More precisely, let us write Definition 2.4.8 of a braided bialgebra diagrammatically, as the commutativity of

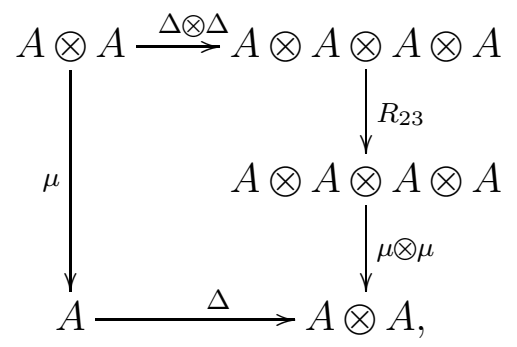

and decompose this condition into parts corresponding to various graded components of $A \otimes A$. That is, we fix $(p, q),(r, s) \in \mathbb{Z}_{+}^{2}$ with $p+q=r+s$ and look at the matrix element of $\Delta \circ \mu$ (the lower left path in the diagram) mapping from $A_{p} \otimes A_{q}$ to $A_{r} \otimes A_{s}$.

Let $S((p, q),(r, s))$ be the set of $2 \times 2$ matrices of nonnegative integers

$$
\left(\begin{array}{ll}
p_{1} & p_{2} \\
q_{1} & q_{2}
\end{array}\right) \text { such that } p_{1}+p_{2}=p, q_{1}+q_{2}=q, \quad p_{1}+q_{1}=r, p_{2}+q_{2}=s .
$$

(This is a particular case of the definition of contingency matrices in $\S 4.1 \mathrm{C}$.) Then (4.2.3) gives

$$
\Delta_{r, s} \circ \mu_{p, q}=\sum_{\left(\begin{array}{cc}
p_{1} & p_{2} \\
q_{1} & q_{2}
\end{array}\right) \in S((p, q),(r, s))}\left(\mu_{p_{1}, q_{1}} \otimes \mu_{p_{2}, q_{2}}\right) \circ\left(\operatorname{Id} \otimes R_{A_{p_{2}}, A_{q_{1}}} \otimes \mathrm{Id}\right) \circ\left(\Delta_{p_{1}, p_{2}} \otimes \Delta_{q_{1}, q_{2}}\right) .
$$


We now reduce our question (that $\delta_{\alpha, \beta}^{\prime}$ commutes with generalization maps) to an instance of (4.2.4). Let $\alpha>_{1} \beta$ and $\mathbf{z} \in X_{\beta}^{\mathfrak{I}}$ be as before. Let us write the action of $\delta_{\alpha, \beta}^{\prime}$ on the stalks at $\mathbf{z}$ more explicitly. By the above assumption (4.2.1), we have $\beta_{i}=\alpha_{i}+\alpha_{i+1}$. Let us write the summand $\mathbf{z}_{i}$ of $\mathbf{z}$ as

$$
\begin{aligned}
\mathbf{z}_{i} & =m_{1} z_{i, 1}+\cdots+m_{l} z_{i, l}, \quad \sum m_{\nu}=\beta_{i}, \\
\operatorname{Im}\left(z_{i, 1}\right) & =\cdots=\operatorname{Im}\left(z_{i, l}\right), \quad \operatorname{Re}\left(z_{i, 1}\right)<\cdots<\operatorname{Re}\left(z_{i, l}\right) .
\end{aligned}
$$

A decomposition $\mathbf{z}_{i}=\mathbf{z}_{i}^{1}+\mathbf{z}_{i}^{2}$ defining a compoment $T\left(\mathbf{z}_{i}^{1}, \mathbf{z}_{i}^{2}\right)$ above, i.e., a summand in $\left(j_{\beta}^{*} æ_{\alpha *} \widetilde{\mathcal{E}}^{\alpha}(A)\right)_{\mathbf{z}}$, is given by writing, in some way,

$$
m_{\nu}=m_{\nu}^{1}+m_{\nu}^{2}, \quad \nu=1, \cdots, l
$$

The corresponding summand is simply

$$
\left(\bigotimes_{m=1}^{i-1} A_{\mathbf{z}_{m}}\right) \otimes \bigotimes_{\nu=1}^{l}\left(A_{m_{\nu}^{1}} \otimes A_{m_{\nu}^{2}}\right) \otimes\left(\bigotimes_{m=i+1}^{k} A_{\mathbf{z}_{m}}\right),
$$

the stalk $\widetilde{\mathcal{E}}^{\beta}(A)_{\mathbf{z}}$ is

$$
\left(\bigotimes_{m=1}^{i-1} A_{\mathbf{z}_{m}}\right) \otimes \bigotimes_{\nu=1}^{l} A_{m_{\nu}} \otimes\left(\bigotimes_{m=i+1}^{k} A_{\mathbf{z}_{m}}\right) .
$$

and the map on the stalks given by $\delta_{\alpha, \beta}^{\prime}$ is

$$
\bigotimes_{\nu=1}^{l}\left(A_{m_{\nu}^{1}} \otimes A_{m_{\nu}^{2}}\right) \stackrel{\mu \otimes \cdots \otimes \mu}{\longrightarrow} \bigotimes_{\nu=1}^{l} A_{m_{\nu}}
$$

tensored on the left and right by the identities. We need to show that such maps commute with the generalization maps for points inside the stratum $X_{\beta}^{\mathfrak{I}}$ (i.e., from a smaller to a bigger fine cell inside this tratum). For this, it suffices to consider elementary generalizations, when the dimension of the fine cell increases by 1 . If we start from $\mathbf{z}$ as before, then an elementary generalization corresponds to splitting just one $m_{j}$, in some way, as $m_{j}^{\prime}+m_{j}^{\prime \prime}$ and moving from $\mathbf{z}$ to a nearby $\widetilde{\mathbf{z}}$ which has the form

$$
\widetilde{\mathbf{z}}=\mathbf{z}_{1}+\cdots+\mathbf{z}_{i-1}+\widetilde{\mathbf{z}}_{i}+\mathbf{z}_{i+1}+\cdots+\mathbf{z}_{k}
$$

where $\mathbf{z}_{t}, t \neq i$ are the same as for $\mathbf{z}$, while $\widetilde{\mathbf{z}}_{i}$ is obtained by splitting some the divisor $m_{j} z_{i, j}$ into $m_{j}^{\prime} z_{i, j}^{\prime}+m_{j}^{\prime \prime} z_{i, j}^{\prime \prime}$ so that

$$
\operatorname{Im}\left(z_{i, j}^{\prime}\right)=\operatorname{Im}\left(z_{i, j}^{\prime \prime}\right)=\operatorname{Im}\left(z_{i, j}\right), \quad \operatorname{Re}\left(z_{i, j}^{\prime}\right)<\operatorname{Re}\left(z_{i, j}^{\prime \prime}\right) .
$$

So the number $m_{j}$ gets split in two ways:

$$
m_{j}=m_{j}^{1}+m_{j}^{2}=m_{j}^{\prime}+m_{j}^{\prime \prime}
$$


We notice that all the tensor products involved will contain the tensor product of the $A_{\mathbf{z}_{m}}$, $m \neq i$, and all the maps involved will be given by identities on these tensor factors. So in the further discussion we will ignore these factors. What will remain is the diagrams of the form

$$
\begin{gathered}
\bigotimes_{\nu=1}^{l}\left(A_{m_{\nu}^{1}} \otimes A_{m_{\nu}^{2}}\right) \longrightarrow\left(\bigotimes_{\nu=1}^{j-1} A_{m_{\nu}^{1}} \otimes A_{m_{\nu}^{2}}\right) \otimes\left(A_{p_{1}} \otimes A_{p_{2}} \otimes A_{q_{1}} \otimes A_{q_{2}}\right) \otimes\left(\bigotimes_{\nu=j+1}^{l} A_{m_{\nu}^{1}} \otimes A_{m_{\nu}^{2}}\right) \\
\quad{ }^{\mu \otimes \cdots \otimes \mu} \\
\bigotimes_{\nu=1}^{l} A_{m_{\nu}} \longrightarrow\left(\bigotimes_{n u=1} A_{m_{\nu}}\right) \otimes\left(A_{m_{j}^{\prime}} \otimes A_{m_{j}^{\prime \prime}}\right) \otimes\left(\bigotimes_{n u=j+1}^{l} A_{m_{\nu}}\right)
\end{gathered}
$$

with

$$
p_{1}+p_{2}=m_{j}^{1}, q_{1}+q_{2}=m_{j}^{2}, \quad p_{1}+q_{1}=m_{j}^{\prime}, p_{2}+q_{2}=m_{j}^{\prime \prime} .
$$

In each such diagram, the left vertical arrow represents (after omitting the inessential tensor factors, as we agreed) the action of $\delta$ on the stalks at $\mathbf{z}$. The lower horizontal arrow represents the generalization map of $\widetilde{\mathcal{E}}^{\beta}(A)$ from $\mathbf{z}$ to $\widetilde{\mathbf{z}}$. The top horizontal arrow, summed over the admissible $p_{1}, p_{2}, q_{1}, q_{2}$, represents the generalization from $\mathbf{z}$ to $\widetilde{\mathbf{z}}$ in $j_{\beta}^{*} j_{\alpha *} \widetilde{\mathcal{E}}^{\alpha}(A)$. The right vertical arrow, similarly summed, represents the action of $\delta_{\alpha, \beta}^{\prime}$ on stalks at $\widetilde{\mathbf{z}}$. So removing even more inessential factors (corresponding to $\nu \neq j$ ), we reduce our statement to (4.2.4). Proposition 4.2.5 is proved.

By adjunction, the morphism $\delta_{\alpha, \beta}^{\prime}$ gives a morphism of sheaves on $\operatorname{Sym}^{n}(\mathbb{C})$

$$
\delta_{\alpha, \beta}: \mathcal{E}^{\alpha}(A) \longrightarrow \mathcal{E}^{\beta}(A), \quad \alpha>_{1} \beta
$$

Proposition 4.2.5. The morphisms $\underline{\delta}_{\alpha, \beta}$ define an anti-representation of $\mathbf{2}^{n-1}$ in the category of sheaves on $\operatorname{Sym}^{n}(\mathbb{C})$, and therefore we have a complex of sheaves

$$
\mathcal{E}_{n}^{\bullet}(A)=\left\{\mathcal{E}^{\left(1^{n}\right)}(A) \stackrel{\underline{\delta}}{\rightarrow} \bigoplus_{l(\alpha)=n-1} \mathcal{E}^{\alpha}(A) \stackrel{\delta}{\rightarrow} \ldots \stackrel{\delta}{\rightarrow} \mathcal{E}^{(n)}(A)\right\}
$$

Set the grading of this complex so that $\mathcal{E}^{1^{n}}(A)$ is situated in degree $(-n)$.

Proof: Follows from the associativity of the multiplication.

We call $\mathcal{E}_{n}^{\bullet}(A)$ the $n$th Cousin complex of $A$. By definition, it is a complex of sheaves on $\operatorname{Sym}^{n}(\mathbb{C})$ constructible with respect to the stratification by fine cells.

Example 4.2.6. Let $n=2$. The complex

$$
\mathcal{E}_{2}^{\bullet}(A)=\left\{\mathcal{E}^{(1,1)}(A) \stackrel{\delta}{\longrightarrow} \mathcal{E}^{(2)}(A)\right\}
$$

consists of 2 terms. After "reducing" (quotienting) by translations, it can be seen as a complex of sheaves on $\mathbb{C}$ stratified by $\mathbb{C} \backslash \mathbb{R}_{\geqslant 0}$, the reduction of $X_{(1,1)}^{\mathfrak{I}}$ and $\mathbb{R}_{\geqslant 0}$, the reduction 
of $X_{(2)}^{\mathfrak{I}}$. The sheaf $\mathcal{E}^{(2)}(A)$ lives on $\mathbb{R}_{\geqslant 0}$ with stalks (at 0 and elsewhere) and the generalization map given by $A_{2} \stackrel{\Delta}{\longrightarrow} A_{1} \otimes A_{1}$.

The sheaf $\mathcal{E}^{(1,1)}(A)$ is the direct image of the local system associated to $A_{1} \otimes A_{1}$ from $\mathbb{C} \backslash \mathbb{R}_{\geqslant 0}$ to the whole of $\mathbb{C}$. So its stalk at 0 is $A \otimes A$ while the stalk at any point of $\mathbb{R}_{>0}$ is the sum of two copies of $A \otimes A$ corresponding to the two sides of the cut $\mathbb{R}_{\geqslant 0}$. The generalization map is given by $(\mathrm{Id}, R)$. Thus the diagram of stalks and generalization maps given by $\underline{\delta}$ over $\mathbb{R}_{\geqslant 0}$ has the form ( $\underline{\delta}$ acts vertically):

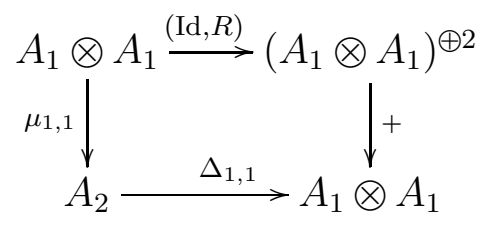

The commutativity of this diagram (i,e., the fact that $\underline{\delta}$ is indeed a morphism of sheaves) is the identity $\Delta_{1,1} \circ \mu_{1,1}=\mathrm{Id}+R$ which is a particular case of (4.2.4), see also (5.2.1) below.

\section{B. Vertical factorizability of Cousin complexes.}

Definition 4.2.7. Let $U_{1}, U_{2} \subset \mathbb{C}$ be open sets. We say that $U_{1}, U_{2}$ are vertically disjoint and write $U_{1}<U_{2}$, if $\operatorname{Im}\left(z_{1}\right)<\operatorname{Im}\left(z_{2}\right)$ for any $z_{1} \in U_{1}, z_{2} \in U_{2}$.

If $U_{1}, U_{2}$ are vertically disjoint, then $U_{1} \cap U_{2}=\varnothing$. As in (3.2.4), we have the addition map

$$
a=\left(a_{p q}\right): \bigsqcup_{p+q=n} \operatorname{Sym}^{p}\left(U_{1}\right) \times \operatorname{Sym}^{q}\left(U_{2}\right) \stackrel{\simeq}{\longrightarrow} \operatorname{Sym}^{n}\left(U_{1} \cup U_{2}\right) .
$$

Proposition 4.2.8. The collection of complexes $\mathcal{E}(A)=\left(\mathcal{E}_{n}^{\bullet}(A)\right)_{n \geqslant 0}$ is vertically factorizable. That is, for any vertically disjoint $U_{1}, U_{2}$ we have an isomorphism of complexes of sheaves

$$
\mu_{U_{1}, U_{2}}^{p q}:\left.\left.\mathcal{E}_{p}^{\bullet}(A)\right|_{\operatorname{Sym}^{p}\left(U_{1}\right)} \bowtie \mathcal{E}_{q}^{\bullet}(A)\right|_{\operatorname{Sym}^{q}\left(U_{2}\right)} \longrightarrow a_{p q}^{*}\left(\left.\mathcal{E}_{n}^{\bullet}(A)\right|_{\operatorname{Sym}^{n}\left(U_{1} \cup U_{2}\right)}\right),
$$

these isomorphisms being compatible with restrictions and associative for any triple $U_{1} \prec$ $U_{2} \prec U_{3}$ of vertically disjoint open sets.

Proof: Let $p+q=n$. For any two ordered partitions

$$
\beta=\left(\beta_{1}, \cdots, \beta_{k}\right) \in 2^{p-1}, \quad \gamma=\left(\gamma_{1}, \cdots, \gamma_{m}\right) \in \mathbf{2}^{q-1}
$$

we denote their concatenation by

$$
\beta \sqcup \gamma=\left(\beta_{1}, \cdots, \beta_{k}, \gamma_{1}, \cdots, \gamma_{m}\right) \in \mathbf{2}^{n-1} .
$$

Our statement follows from the identifications of sheaves

$$
\mu_{U_{1}, U_{2}}^{\beta, \gamma}:\left.\left.\mathcal{E}^{\beta}(A)\right|_{\operatorname{Sym}^{p}\left(U_{1}\right)} \bowtie \mathcal{E}^{\gamma}(A)\right|_{\operatorname{Sym}^{q}\left(U_{2}\right)} \stackrel{\simeq}{\longrightarrow} a_{p q}^{*}\left(\left.\mathcal{E}^{\beta \sqcup \gamma}(A)\right|_{\left.\operatorname{Sym}^{n}\left(U_{1}\right) \cup U_{2}\right)}\right) .
$$

To construct these, we recall that $\mathcal{E}^{\beta}(A)$ is defined as the direct image of the sheaf $\widetilde{\mathcal{E}}^{\beta}(A)$ from the imaginary stratum $X_{\beta}^{\mathfrak{I}}$, and similarly for $\mathcal{E}^{\gamma}(A)$. More precisely, the construction of $\mu_{U_{1}, U_{2}}^{\beta, \gamma}$ follows from: 
(1) The equality of subsets

$$
\left(X_{\beta}^{\mathfrak{I}} \cap \operatorname{Sym}^{p}\left(U_{1}\right)\right) \times\left(X_{\gamma}^{\mathfrak{I}} \cap \operatorname{Sym}^{q}\left(U_{2}\right)\right)=a_{p q}^{-1}\left(X_{\beta \sqcup \gamma}^{\mathfrak{I}} \cap \operatorname{Sym}^{n}\left(U_{1} \cup U_{2}\right)\right),
$$

and

(2) The identification of two sheaves on the space in (1):

(2L) The restriiction of $\widetilde{\mathcal{E}}^{\beta}(A) \bowtie \widetilde{\mathcal{E}}^{\gamma}(A)$ to the LHS in (1), and

(2R) The pullback, under $a_{p q}$, of th restriction of $\widetilde{\mathcal{E}}^{\beta \sqcup \gamma}(A)$ to the RHS in (1).

The equality (1) follows from the very definition of the imaginary strata, while the identification (2) follows from the very definition of the sheaves $\widetilde{\mathcal{E}}^{\alpha}$.

C. Stalks of Cousin complexes. Recall the notations $B_{n}(A)$ for the $n$th bar-complexe of $A$. For any point $\mathbf{z}=\sum_{i=1}^{m} \lambda_{i} z_{i} \in \operatorname{Sym}^{n}(\mathbb{C})$ we denote

$$
B_{\mathbf{z}}(A)=\bigotimes_{\left(z_{1}, \cdots, z_{m}\right)} B_{\lambda_{i}}(A)
$$

Proposition 4.2.9. (a) The complex $\mathcal{E}_{n}^{\bullet}(A)$ is constructible with respect to the diagonal stratification $S$ of $\operatorname{Sym}^{n}(\mathbb{C})$ by complex strata $X_{\lambda}^{\mathbb{C}}$.

(b) In fact, the stalk of $\mathcal{E}_{n}^{\bullet}(A)$ at a point $\mathbf{z} \in \operatorname{Sym}^{n}(\mathbb{C})$ is quasi-isomorphic to $B_{\mathbf{z}}(A)$.

The proof of Proposition 4.2.9 will occupy the rest of this $\S \mathrm{C}$. Before giving the proof in general, we consider an example which illustrates the situation.

Example 4.2.10. Proposition 4.2.9 means, in particular, that the restriction of $\mathcal{E}_{n}^{\bullet}(A)$ to the generic stratum $\operatorname{Sym}_{\neq}^{n}(\mathbb{C})=X_{\left(1^{n}\right)}^{\mathbb{C}}$ is quasi-isomorphic to $\mathcal{L}_{1^{n}}(A)[n]$, the local system of determinations of $A_{1}^{\otimes n}$, put in degree $(-n)$. However, the stalk $\mathcal{E}_{n}^{\bullet}(A)_{\mathbf{z}}, \mathbf{z} \in \operatorname{Sym}_{\neq}^{n}(\mathbb{C})$, as a complex, depends on the Fox-Neuwirth cell $X_{\left[\beta: 1^{n}\right]}$ in which $\mathbf{z}$ lies. Let us see why, up to quasi-isomorphism, the answer is always the same, namely the stalk of $\mathcal{L}_{1^{n}}(A)[n]$.

The simplest case is when $\beta=\left(1^{n}\right)$, i.e., $\operatorname{Im}\left(z_{1}\right)<\cdots<\operatorname{Im}\left(z_{n}\right)$ after a reordering. In this case only the sheaf $\mathcal{E}^{\left(1^{n}\right)}(A)$ has non-trivial stalk at $\mathbf{z}$ (the other sheaves being supported on higher codimension strata), and this stalk is $A_{1}^{\otimes n}$. So in this case $\mathcal{E}^{\bullet}(A)_{\mathbf{z}}=A_{\mathbf{z}}[n]=B_{\mathbf{z}}(A)$ as a complex.

The most complicated case is when $\beta=(n)$, i.e., $\operatorname{Im}\left(z_{1}\right)=\cdots=\operatorname{Im}\left(z_{n}\right)$, and we can assume $\operatorname{Re}\left(z_{1}\right)<\cdots<\operatorname{Re}\left(z_{n}\right)$ after a reordering. In this case all the $\mathcal{E}^{\alpha}(A)$ will have nontrivial stalks at $\mathbf{z}$. The stalk of a given $\mathcal{E}^{\alpha}(A)$ is, by Proposition 4.1.12, the sum of as many copies of $A_{1}^{\otimes n}$, as there are contingency matrices $\zeta$ of type $\left(\alpha,\left(1^{n}\right)\right)$. Such matrices are, by Example, 4.1.8, in bijection with shuffles of type $\alpha$, i.e., with sequences $\left(I_{1}, \cdots, I_{l}\right)$ of subsets of $\{1, \cdots, n\}$ forming a disjoint decomposition and such that $\left|I_{\nu}\right|=\alpha_{\nu}$. Such a 
sequence gives a face $\left[I_{1}, \cdots, I_{l}\right]$ of the $n$th permutohedron $P_{n}$, see Proposition A.1. When $\alpha$ and $\zeta$ vary, we get precisely all the faces. Thus the stalk at $\mathbf{z}$, as a complex, is:

$$
\mathcal{E}^{\bullet}(A)_{\mathbf{z}}=A_{1}^{\otimes n}[n] \otimes_{\mathbf{k}} C^{\bullet}\left(P_{n}, \mathbf{k}\right),
$$

where $C^{\bullet}\left(P_{n}, \mathbf{k}\right)$ is the cellular cochain complex of $P_{n}$ (with respect to the CW-decomposition given by the faces) with coefficients in $\mathbf{k}$. Since $P_{n}$, being a convex polytope, is contractible, $C^{\bullet}\left(P_{n}, \mathbf{k}\right)$ is quasi-isomorphic to $\mathbf{k}$ in degree 0 , which gives our desired quasi-isomorphism for $\mathcal{E}^{\bullet}(A)_{\mathbf{z}}$.

Proof of Proposition 4.2.9: We will prove (a) and (b) simultaneously. To prove (a) we need to show that for any $\lambda \in \mathcal{P}(n)$, any $\mathbf{z} \in X_{\lambda}^{\mathbb{C}}$ and any nearby $\mathbf{z}^{\prime}$ lying in the same $X_{\lambda}^{\mathbb{C}}$, the generalization map $\gamma_{\mathbf{z}, \mathbf{z}^{\prime}}: \mathcal{E}_{n}^{\bullet}(A)_{\mathbf{z}} \rightarrow \mathcal{E}_{n}^{\bullet}(A)_{\mathbf{z}^{\prime}}$ is a quasi-isomorphism. So let $\mathbf{z}$ be fixed.

Let $\beta \leqslant \gamma$ be such that $\mathbf{z} \in X_{[\beta: \gamma]}$. We notice first that because of vertical factorizability of $\mathcal{E}^{\bullet}(A)$, both our statements (quasi-isomorphicity of $\gamma_{\mathbf{Z}^{\prime}}$ for nearby $\mathbf{z}^{\prime} \in X_{\lambda}^{\mathbb{C}}$ as well as the identification of $\left.\mathcal{E}_{n}^{\bullet}(A)_{\mathbf{z}}\right)$ reduce to the case where $\beta=(n)$, i.e., $\operatorname{Im}\left(z_{1}\right)=\cdots=\operatorname{Im}\left(z_{m}\right)$. Ordering the $z_{i}$ according to the increasing real part, we see that $z_{i}$ enters into $\mathbf{z}$ with multiplicity $\gamma_{i}$, i.e., $\lambda=\bar{\gamma}$ is the unordered partition of $n$ associated to then ordered partition $\gamma$. This means that

$$
B_{\mathbf{z}}(A)=B_{\gamma}(A)=B_{\gamma_{1}}(A) \otimes \cdots \otimes B_{\gamma_{m}}(A)
$$

(isomorphism of complexes of objects of $\mathcal{V}$ ). Further, let us write each bar-complex $B_{\gamma_{i}}(A)$ as a sum of explicit summands, i.e., identify, as a graded object:

$$
B_{\gamma_{i}}(A)=\underset{\theta^{(i)}=\left(\theta_{1}^{(i)}, \cdots, \theta_{m_{i}}^{(i)}\right) \in \mathbf{2}^{\gamma_{i}}}{A_{\theta^{(i)}}}\left[m_{i}\right], \quad A_{\theta^{(i)}}=A_{\theta_{1}^{(i)}} \otimes \cdots \otimes A_{\theta_{m_{i}}^{(i)}}, \quad m_{i}=l\left(\theta^{(i)}\right) .
$$

Thus, as a graded object,

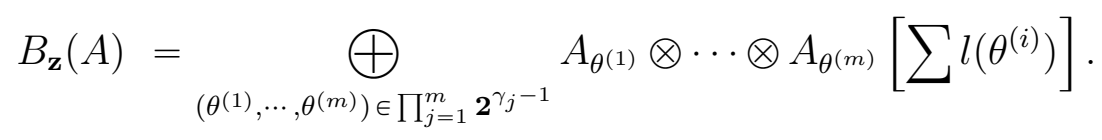

We compare this with the shape of the stalk $\mathcal{E}_{n}^{\bullet}(A)_{\mathbf{z}}$. By Proposition 4.1.12, it is a certain direct sum: first, over $\alpha \in \mathbf{2}^{n-1}$ (parametrizing the individual $\mathcal{E}^{\alpha}(A)$ ) and, second, over contingency matrices $\zeta \in S(\alpha, \gamma)$ (parametrizing the summands in the stalk of $\mathcal{E}^{\alpha}(A)$ at $\mathbf{z}$ ). For such $\zeta=\left\|\zeta_{i j}\right\|$ we denote by $\zeta_{\bullet j}$ the $j$ th column of $\zeta$. This is a vector in $\mathbb{Z}_{\geqslant 0}^{l(\alpha)}$ with entries summing up to $\gamma_{j}$, with some of these entries possibly being zero. We denote by $\mathbf{c}_{j}(\zeta)$ and call the reduced $j$ th column of $\zeta$ the ordered partition of $\gamma_{j}$ obtained by removing the zeroes from the entries of $\zeta_{\bullet j}$. The summand corresponding to $\alpha$ and $\zeta$ is, by Proposition 4.1.12, the tensor product $A_{\zeta}$ which we can canonically identify as

$$
A_{\zeta}=A_{\mathbf{c}_{1}(\zeta)} \otimes \cdots \otimes A_{\mathbf{c}_{m}(\zeta)}
$$

So as a graded object,

$$
\mathcal{E}_{n}^{\bullet}(A)_{\mathbf{z}}=\bigoplus_{\alpha \in \mathbf{2}^{n-1}} \bigoplus_{\zeta \in S(\alpha, \gamma)} A_{\mathbf{c}_{1}(\zeta)} \otimes \cdots \otimes A_{\mathbf{c}_{m}(\zeta)}[l(\alpha)]
$$


This means that each summand $A_{\theta^{(1)}} \otimes \cdots \otimes A_{\theta^{(m)}}$ in (4.2.11) will be found in (4.2.12) possibly several times: once for each occurrence of $\alpha \in \mathbf{2}^{n-1}$ and $\zeta \in S(\alpha, \gamma)$ such that $\mathbf{c}_{j}(\zeta)=\theta^{(j)}$ for all $j=1, \cdots, m$.

The possible $\zeta$ with this property are in bijection with XO-tables of type $\left(l\left(\theta^{(1)}\right), \cdots, l\left(\theta^{(m)}\right)\right.$, see Definition A.10. Indeed, all we need to do to recover $\zeta$ is, for any $j$, to mark by $\mathrm{X}$ the positions in the $j$ th column which will be occupied by nonzero entries of $\theta^{(j)}$. There are $l\left(\theta^{(j)}\right)$ such positions. Once we know $\zeta$, the partition $\alpha$ is found uniquely by summing the rows. This implies that at the level of graded objects, we have an identification

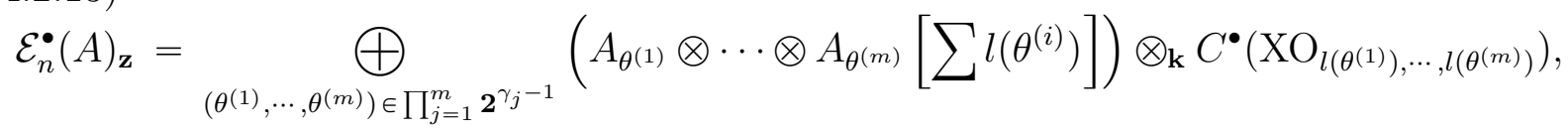

where $C^{\bullet}\left(\mathrm{XO}_{l\left(\theta^{(1)}\right), \cdots, l\left(\theta^{(m)}\right)}\right)$ is the XO-cochain complex, see Definition A.14, and $\otimes_{\mathbf{k}}$ means the tensor product of a (graded) object of $\mathcal{V}$ and a (graded) $\mathbf{k}$-vector space given by the k-linear structure on $\mathcal{V}$.

We next define a morphism of graded objects

$$
\gamma: \mathcal{E}_{n}^{\bullet}(A)_{\mathbf{z}} \longrightarrow B_{\mathbf{z}}(A)
$$

in terms of decompositions (4.2.13) and (4.2.11). For every $\left(\theta^{(1)}, \cdots, \theta^{(m)}\right) \in \prod_{j=1}^{m} \mathbf{2}^{\gamma_{j}-1}$ we consider the morphism of complexes

$$
\begin{gathered}
q_{\theta^{(1)}, \cdots, \theta(m)}=\Sigma \circ p, \\
C^{\bullet}\left(\mathrm{XO}_{l\left(\theta^{(1)}\right), \cdots, l\left(\theta^{(m)}\right)}\right) \stackrel{p}{\longrightarrow} C^{0}\left(\mathrm{XO}_{l\left(\theta^{(1)}\right), \cdots, l\left(\theta^{(m)}\right)}\right) \stackrel{\Sigma}{\longrightarrow} \mathbf{k},
\end{gathered}
$$

where $p$ is the projection to the degree 0 component and $\Sigma$ is given by summing the values of a 0-cochain over all the vertices of the XO-complex. We then define $\gamma$ to act om the summand in (4.2.13) corresponding to $\left(\theta^{(1)}, \cdots, \theta^{(m)}\right)$ as $\operatorname{Id} \otimes_{\mathbf{k}} q_{\theta^{(1)}, \cdots, \theta^{(m)}}$.

We claim that $\gamma$ is in fact a morphism of complexes. This can either be seen directly by analyzing the differential in $\mathcal{E}_{n}^{\bullet}(A)_{\mathbf{z}}$ (described below) or, more conveniently for us, deduced from the following observation:

$\gamma$ is a particular instance of the generalization maps $\gamma_{\mathbf{z}, \mathbf{z}^{\prime}}$ where $\mathbf{z}^{\prime}$ is a small displacement of $\mathbf{z}$ in the same stratum $X_{\lambda}^{\mathbb{C}}$.

More precisely, $\gamma$ corresponds to the case when $\mathbf{z}^{\prime}$ is a maximally generic displacement of $\mathbf{z}$ inside $X_{\lambda}^{\mathbb{C}}$, that is $\mathbf{z}^{\prime} \in X_{[\gamma: \gamma]}$, while $\mathbf{z} \in X_{[(n): \gamma]}$. In other words, assume that the displacement is of the form

$$
\mathbf{z}=\sum_{i=1}^{m} \gamma_{i} z_{i} \quad \leadsto \quad \mathbf{z}^{\prime}=\sum_{i=1}^{m} \gamma_{i} z_{i}^{\prime}, \quad \operatorname{Im}\left(z_{1}^{\prime}\right)<\cdots<\operatorname{Im}\left(z_{m}^{\prime}\right)
$$

(we move each $z_{i}$ in the vertical direction a bit to make the imaginary parts all different). In this case, similarly to Example 4.2.10,

$$
\mathcal{E}_{n}^{\bullet}(A)_{\mathbf{z}^{\prime}}=B_{\mathbf{z}^{\prime}}(A)
$$


as a complex. If we identify $B_{\mathbf{z}^{\prime}}(A)$ with $B_{\mathbf{z}}(A)$ by continuity of the braided tensor prouct $\bigotimes_{\left(z_{1}, \cdots, z_{m}\right)}^{(m)}$ under displacement of the points, then the map $\gamma_{\mathbf{z}, \mathbf{z}^{\prime}}$ becomes precisely $\gamma$. Since we known that each $\gamma_{\mathbf{z}, \mathbf{z}^{\prime}}$ is a morphism of complexes, so is $\gamma$.

We now analyze the differential in $\mathcal{E}_{n}^{\bullet}(A)_{\mathbf{z}}$. Fix $\alpha \in \mathbf{2}^{n-1}$ and $\zeta \in S(\alpha, \gamma)$, so that the corresponding summand in $\mathcal{E}_{n}^{\bullet}(A)_{\mathbf{z}}$ is $A_{\zeta}$. Let $\zeta_{\nu, \bullet}$ be the $\nu$ th row of $\zeta$. (After omitting the zeroes, $\zeta_{\nu, \bullet}$ becomes an ordered partition of $\left.\alpha_{\nu}\right)$. As before, let also $\theta^{(j)}=\mathbf{c}_{j}(\zeta)$ be the ordered partition of $\gamma_{j}$ obtained by omitting zeroes of the column $\zeta_{\bullet, j}$.

Further let $\beta \in \mathbf{2}^{n-1}$ be such that $\alpha>_{1} \beta$ so $\beta$ is obtained by combining some $\alpha_{i}$ and $\alpha_{i+1}$ together, as in (4.2.1). Let us look at the action of $\delta_{\alpha, \beta}: \mathcal{E}^{\alpha}(A)_{\mathbf{z}} \rightarrow \mathcal{E}^{\beta}(A)_{\mathbf{z}}$ on the summand $A_{\zeta}$. This action consists of a morphism $\delta_{\zeta, \zeta^{\prime}}: A_{\zeta} \rightarrow A_{\zeta^{\prime}}$, where $\zeta^{\prime} \in S(\beta, \gamma)$ is obtained by adding together the rows $\zeta_{i, \bullet}$ and $\zeta_{i+1, \bullet}$ and keeping the other rows of $\zeta$ intact. Now, there are the following possibilities:

(0) The rows $\zeta_{i, \bullet}$ and $\zeta_{i+1, \bullet}$ have disjoint sets of X's (i.e., of positions filled with non-zero entries).

(1) $\zeta_{i, \bullet}$ and $\zeta_{i+1, \bullet}$ have exactly one X-position in common.

$\left(2^{+}\right) \zeta_{i, \bullet}$ and $\zeta_{i+1, \bullet}$ have some number $k \geqslant 2 \mathrm{X}$-positions in common.

In the case (0) we have $A_{\zeta^{\prime}}=A_{\zeta}$ and $\delta_{\zeta, \zeta^{\prime}}= \pm \mathrm{Id}$. This is a matrix element of the differential $d_{\mathrm{XO}}$ in $C^{\bullet}\left(\mathrm{XO}_{l\left(\theta^{(1)}, \ldots, l\left(\theta^{(m)}\right)\right.}\right.$ multiplied by the identity of the first factor in the corresponding summand in (4.2.13).

In the cases $(1)$ and $\left(2^{+}\right)$the differential $\delta_{\zeta, \zeta^{\prime}}$ is given by the multiplication in $A$. But the difference between them is as follows. In the case (1) we have a matrix element of the differential $d_{B}$ in $B_{\mathbf{z}}(A)=\otimes B_{\gamma_{\nu}}(A)$ (tensored by the identity of $C^{\bullet}\left(\mathrm{XO}_{l\left(\theta^{(1)}, \cdots, l\left(\theta^{(m)}\right)\right.}\right)$ ). That last differential is the tensor products of the differentials in the $B_{\gamma_{\nu}}(A)$ and so each matrix element of it acts by increasing the degree by 1 in just a single tensor factor $B_{\gamma_{\nu}}(A)$, leaving the other tensor factors intact. In contrast, in the case $\left(2^{+}\right)$the matrix element, while having a similar general nature (multiplying some groups of components of $A$ together) acts simultaneously on more than one tensor factor constituting $B_{\mathbf{z}}(A)$.

To account for this difference, we note that the decomposition (4.2.13) gives a bigrading of $\mathcal{E}_{n}^{\bullet}(A)_{\mathbf{z}}$, and $\delta_{\zeta, \zeta^{\prime}}$ has, with respect to this bigrading, the bidegree $(1-k, k)$ in all cases $(k=0,1,2, \cdots)$. So considering the filtration of (4.2.13) whose $d$ th layer is the sum of the

$$
\left(A_{\theta^{(1)}} \otimes \cdots \otimes A_{\theta^{(m)}}\left[\sum l\left(\theta^{(i)}\right)\right]\right)^{\geqslant d} \otimes_{\mathbf{k}} C^{\bullet}\left(\mathrm{XO}_{l\left(\theta^{(1)}\right), \cdots, l\left(\theta^{(m)}\right)}\right),
$$

we see that the quotients of the filtration are the complexes $C^{\bullet}\left(\mathrm{XO}_{l\left(\theta^{(1)}, \cdots, l\left(\theta^{(m)}\right)\right.}\right)$ tensored with various homogeneous parts of $A_{\theta^{(1)}} \otimes \cdots \otimes A_{\theta^{(m)}}$ (the latter considered as graded objects with no differential). Since $C^{\bullet}\left(\mathrm{XO}_{l\left(\theta^{(1)}\right), \cdots, l\left(\theta^{(m)}\right)}\right)$ is, by Proposition A.15, quasi-isomorphic to $\mathbf{k}$ in degree 0, the morphism $q_{\theta^{(1)}, \ldots, \theta^{(m)}}$ in (4.2.14) is a quasi-isomorphism (here we use the assumptions $\operatorname{char}(\mathbf{k})=0$ ). Therefore the associated graded object, i.e., the term $E_{1}$ of 
the spectral sequence of our filtered complex, is identified with $B_{\mathbf{z}}(A)$, and the differential $d_{1}$ is the differential in $B_{\mathbf{z}}(A)$ (coming from Case (1)). We note further that $\gamma$ is a morphism of filtered complexes if we consider the "stupid filtration" of the target by the $B_{\mathbf{z}}(A) \geqslant j$ and so gives a morphism of the spectral sequences associated to the source and target. By what we shown, this morphism of spectral sequences gives an isomorphism of the $E_{1}$-terms so it is an isomorphism, and therefore $\gamma$ is a quasi-isomorphism. In particular, $\mathcal{E}_{n}^{\bullet}(A)_{\mathbf{z}}$ is quasi-isomorphic to $B_{\mathbf{z}}(A)$. This proves part (a) of Proposition 4.2.9.

Let us prove part (b). This part means that each generalization map $\gamma_{\mathbf{z}, \mathbf{z}^{\prime}}$ where $\mathbf{z}, \mathbf{z}^{\prime}$ lie in the same complex stratum $X_{\lambda}$, is a quasi-isomorphism. Now, by the transitive property of the generalization maps, it is enough to establish quasi-isomorphicity for the case when $\mathbf{z}^{\prime}$ is the maximally generic displacement within $X_{\lambda}$, considered above. In this case, as we have seen, $\gamma_{\mathbf{z}, \mathbf{z}^{\prime}}$ is identified with the map $\gamma$ which we have just shown to be a quasi-isomorphism. Proposition 4.2.9 is proved.

D. Costalks of Cousin complexes. We now identify the costalks of $\mathcal{E}_{n}^{\bullet}(A)$, i.e., the complexes of vector spaces $i_{\mathbf{z}}^{!} \mathcal{E}_{n}^{\bullet}(A), \mathbf{z}=\sum_{i=1}^{m} \lambda_{i} z_{i} \in \operatorname{Sym}^{n}(\mathbb{C})$. Here $i_{\mathbf{z}}:\{\mathbf{z}\} \rightarrow \operatorname{Sym}^{n}(\mathbb{C})$ is the embedding of $\mathbf{z}$.

Recall that $B_{n}^{\dagger}(A)$ is the $n$th cobar-complex of $A$. As before, we write

$$
B_{\mathbf{z}}^{\dagger}(A)=\bigotimes_{\left(z_{1}, \cdots, z_{m}\right)} B_{\lambda_{i}}^{\dagger}(A)
$$

Proposition 4.2.15. The costalk of $\mathcal{E}_{n}^{\bullet}(A)$ at a point $\mathbf{z} \in \operatorname{Sym}^{n}(\mathbb{C})$ is quasi-isomorphic to $B_{\mathbf{z}}^{\dagger}(A)$.

Proof: As with Proposition 4.2.9, we assume that $\mathbf{z} \in X_{[\beta: \gamma]}$. Vertical factorizability of the $\mathcal{E}_{n}^{\bullet}(A)$ reduces our statement to the case $\beta=(n)$. That is, $\mathbf{z} \in X_{(n)}^{\mathfrak{I}}$ lies in the minimal imaginary stratum. Recall that $X_{(n)}^{\mathfrak{I}}$ consists of $\mathbf{z}$ such that $\operatorname{Im}\left(z_{1}\right)=\cdots=\operatorname{Im}\left(z_{m}\right)$, or, put differently, $\mathbf{z}$ is obtained by a translation, by a purely imaginary number, from a divisor in $K_{n}=\operatorname{Sym}^{n}(\mathbb{R})$. This means that we have an identification $X_{(n)}^{\mathfrak{I}} \simeq K_{n} \times i \mathbb{R}$. Let $\rho: X_{(n)}^{\mathfrak{I}} \longrightarrow$ $K_{n}$ be the projection.

We look at the costalks at $\mathbf{z}$ of individual terms $\mathcal{E}^{\alpha}(A)$ of the complex $\mathcal{E}_{n}^{\bullet}(A)$. Recall that we denote by $j_{\alpha}: X_{\alpha}^{\mathfrak{I}} \rightarrow \operatorname{Sym}^{n}(\mathbb{C})$ the embedding of the imaginary stratum corresponding to $\alpha$.

If $\alpha \neq(n)$, then $\mathbf{z}$ lies in the closure of $X_{\alpha}^{\mathfrak{I}}$ but not in $X_{\alpha}^{\mathfrak{I}}$ itself. This implies that the costalk at $\mathbf{z}$ of $\mathcal{E}^{\alpha}(A)=j_{\alpha_{*}} \widetilde{\mathcal{E}}^{\alpha}(A)$ is 0 by the base change theorem.

If $\alpha=(n)$, then $\mathcal{E}^{(n)}(A)$ is already supported on the closed stratum $X_{(n)}^{\mathfrak{I}}$. Denoting, by a minor abuse of notation, the embedding $\{\mathbf{z}\} \rightarrow X_{(n)}^{\mathfrak{I}}$ by the same symbol $i_{\mathbf{z}}$, we have

$$
i_{\mathbf{z}}^{!} \mathcal{E}^{(n)}(A)=i_{\mathbf{z}}^{!}\left(j_{(n)}^{!} \mathcal{E}^{(n)}(A)\right)
$$

The sheaf $j_{(n)}^{!} \mathcal{E}^{(n)}(A)$ on $X_{(n)}^{\mathfrak{I}}$ coincides wth $j_{(n)}^{*} \mathcal{E}^{(n)}(A)$ and is what we denoted earlier (4.1.5) by $\widetilde{\mathcal{E}}^{(n)}(A)$. So it has the form $\rho^{*} R_{n}(A)$, where $R_{n}(A)$ is the constructible sheaf on $K_{n}$ corresponding to $\mathbb{B}_{n}^{\dagger}(A)$, the $n$th cobar-cube of $A$. 
Now, $K_{n}$ with its stratification $S_{\mathbb{R}}$ and $X_{(n)}^{\mathfrak{I}}$ with the pullback stratification are quasiregular cell complexes (see Definition 1.1.9), and $R_{n}(A)$ and, therefore, $\mathcal{E}_{n}^{(n)}(A)$ are cellular sheaves on them. We now recall the following well known fact.

Lemma 4.2.16. Let $Y$ be a quasi-regular cell complex and $\mathcal{F}$ be a cellular sheaf on $Y$. For a cell $\sigma$ of $Y$ let $j_{\sigma}: \sigma \rightarrow Y$ be the embedding. Denote the stalk of $\mathcal{F}$ at $\sigma$ by $F_{\sigma}=\Gamma\left(\sigma, j_{\sigma}^{*} \mathcal{F}\right)$. Then the costalk $\Gamma\left(\sigma, j_{\sigma}^{!} \mathcal{F}\right)$ at $\sigma$ is quasi-isomorphic to the complex

$$
F_{\sigma} \stackrel{\gamma}{\longrightarrow} \bigoplus_{\substack{\tau \supset \sigma \\ \operatorname{dim}(\tau=\operatorname{dim}(\sigma)+1}} F_{\tau} \otimes \mathrm{OR}(\tau / \sigma) \stackrel{\gamma}{\longrightarrow} \bigoplus_{\substack{\tau \supset \sigma \\ \operatorname{dim}(\tau=\operatorname{dim}(\sigma)+2}} F_{\tau} \otimes \mathrm{OR}(\tau / \sigma) \stackrel{\gamma}{\longrightarrow} \cdots
$$

where $\operatorname{OR}(\tau / \sigma)$ is the 1-dimensional relative orientation $\mathbf{k}$-vector space, and the differential $\gamma$ is induced by the generalization maps of $\mathcal{F}$. The grading of this complex is normalized so that $F_{\sigma}$ is in degree 0 .

We apply this lemma to $\mathcal{F}=\widetilde{\mathcal{E}}^{(n)}(A)$. The cells in $X_{(n)}^{\mathfrak{I}}$ are the fine cells $X_{[(n): \gamma]}$. If $\mathbf{z} \in X_{[(n): \gamma]}$ as we assumed, then the complex of the lemma will be precisely $B_{\mathbf{z}}(A)$ but with a shift: the grading will start from degree 0 on the left. This is because it describes the stalk at the cell (i.e., $j_{\sigma}^{!}$instead of $j_{\mathbf{z}}^{!}$). Passing from that to the stalk at $\mathbf{z}$ itself removes the shift (by introducing the cohomology of a constant sheaf on the cell with support at $\mathbf{z}$ ) and we get $B_{\mathbf{z}}(A)$ with correct grading.

\section{E. Perversity of Cousin complexes.}

Proposition 4.2.17. The complex $\mathcal{E}_{n}^{\bullet}(A)$ is a perverse sheaf constructible with respect to the stratification $S$, i.e., an object of $\operatorname{Perv}\left(\operatorname{Sym}^{n}(\mathbb{C}), S, \mathcal{V}\right)$.

Proof: The $S$-constructibility is shown in Proposition 4.2.9. So it remains to prove the perversity conditions $\left(P^{+}\right)$and $\left(P^{-}\right)$, see $\S 1.1 \mathrm{~A}$.

We start with $\left(P^{+}\right)$. As the stalk $i_{\mathbf{z}}^{*} \mathcal{E}_{n}^{\bullet}(A)$ is quasi-isomorphic to $B_{\mathbf{z}}(A)$ (Proposition 4.2.9), we need to show that the locus of $\mathbf{z}$ for which $H^{-j}\left(B_{\mathbf{z}}(A)\right) \neq 0$, has complex dimension $\leqslant j$. Indeed, suppose $\mathbf{z} \in X_{\lambda}^{\mathbb{C}}$, so $\mathbf{z}=\sum_{i=1}^{m} \lambda_{i} z_{i}$ with $z_{i}$ distinct. By definition, $B_{\mathbf{z}}(A)$ is the tensor product of the $B_{\lambda_{i}}(A)$, and each $B_{\lambda_{i}}(A)$ is a complex concentrated in degrees fron $\left(-\lambda_{i}\right)$ to $(-1)$. So $B_{\mathbf{z}}(A)$ is concentrated in degrees from $(-n)$ to $(-m)$. Now, the complex dimension of $X_{\lambda}^{\mathbb{C}}$ is $m$, and $\left(P^{+}\right)$follows.

The condition $\left(P^{-}\right)$follows similarly from Proposition 4.2.15.

\subsection{Factorizability of Cousin complexes: proof of Theorem 3.3.1}

A. From vertical factorizability to full factorizability. Recall (§3.2) that the space $E_{2}(m)$ of the $E_{2}$-operad consists of $m$-tuples $\left(U_{1}, \cdots, U_{m}\right)$ of disjoint round disks in the unit disk $D \subset \mathbb{C}$. Let $E_{2}^{<}(n) \subset E_{2}(m)$ be the subspace formed by $\left(U_{1}, \cdots, U_{m}\right)$ such that $U_{1} \prec \cdots<U_{m}$, see Definition 4.2.7. The space $E_{2}^{<}(n)$ is contractible and together they 
form a (non-symmetric) suboperad $E_{2}^{<}$in $E_{2}$. Thus $E_{2}^{<}$is a non-symmetric verstion of the homotopy associative operad $E_{1}$.

Let $A$ be a primitive bialgebra in $\mathcal{V}$, as before. We want to equip the collection of perverse sheaves $\mathcal{E}(A)=\left(\mathcal{E}_{n}^{\bullet}(A)\right)$ with a structure of a factorizable perverse sheaf in the sense of Definition 3.2.5. That is, for each $m \geqslant 0$ and each $\left(U_{1}, \cdots U_{m}\right) \in E_{2}(m)$, we want to construct an isomorphism of perverse sheaves (i.e, an isomorphism of the objects of the derived category)

$$
\mu_{U_{1}, \cdots, U_{m}}:\left.\mathrm{X}_{\left(U_{1}, \cdots, U_{m}\right)} \mathcal{F}\right|_{\operatorname{Sym}\left(U_{i}\right)} \longrightarrow a^{*}\left(\left.\mathcal{F}\right|_{\operatorname{Sym}\left(\cup U_{i}\right)}\right)
$$

so that these isomorphisms are compatible with the operadic compositions.

For $\left(U_{1}, \cdots, U_{m}\right) \in E_{2}^{<}(m)$, such an isomorphism is already given by Proposition 4.2.8. In this case $\mu_{U_{1}, \cdots, U_{m}}$ is in fact an isomorphism of complexes of sheaves. These isomorphisms are compatible with the operadic compositions in $E_{2}^{<}$.

Next, we want to define the isomorphisms $\mu_{U_{1}^{\prime}, \cdots, U_{m}^{\prime}}$ for any $\left(U_{1}^{\prime}, \cdots, U_{m}^{\prime}\right) \in E_{2}(m)$ by extending them from $E_{2}^{<}(m)$ to $E_{2}(m)$ "by continuity". That is, let

$$
\gamma:[0,1] \longrightarrow E_{2}(m), \quad t \mapsto \gamma(t)=\left(U_{1}(t), \cdots, U_{m}(t)\right)
$$

be a continuous path in $E_{2}(m)$. We denote $U_{i}=U_{i}(0)$ and $U_{i}^{\prime}=U_{i}(1)$. Let also $U(t)=$ $\bigcup U_{i}(t)$ and $U=U(0), U^{\prime}=U(1)$.

Because perverse sheaves are a purely topological concept, the 1-parameter family of disks $U_{i}(t)$ defines a 1-parameter family of equivalences of categories ("isomonodromic deformations of perverse sheaves")

$$
h_{t}: \operatorname{Perv}\left(\operatorname{Sym}\left(U_{i}(0), S\right) \longrightarrow \operatorname{Perv}\left(\operatorname{Sym}\left(U_{i}(t)\right), S\right) .\right.
$$

Moreover, if $\mathcal{F} \in \operatorname{Perv}(\operatorname{Sym}(D), S)$, then $h_{t}$ takes the restriction $\left.\mathcal{F}\right|_{\operatorname{Sym}\left(U_{0}\right)}$ to $\left.\mathcal{F}\right|_{\operatorname{Sym}\left(U_{t}\right)}$. We have a similar family of equivalences of categories for the unions

$$
k_{t}: \operatorname{Perv}(\operatorname{Sym}(U(0), S) \longrightarrow \operatorname{Perv}(\operatorname{Sym}(U(t)), S) .
$$

Taking $t=1$, we obtain the isomorphism ("monodromy map")

$M_{\gamma}: \operatorname{Hom}\left(\left.\bigotimes_{\left(U_{1}, \cdots, U_{m}\right)} \mathcal{F}\right|_{\operatorname{Sym}\left(U_{i}\right)}, a^{*}\left(\left.\mathcal{F}\right|_{\operatorname{Sym}(U)}\right)\right) \rightarrow \operatorname{Hom}\left(\left.\bigotimes_{\left(U_{1}^{\prime}, \cdots, U_{m}^{\prime}\right)} \mathcal{F}\right|_{\operatorname{Sym}\left(U_{i}^{\prime}\right)}, a^{*}\left(\left.\mathcal{F}\right|_{\operatorname{Sym}\left(U^{\prime}\right)}\right)\right)$.

These isomorphisms are compatible with concatenation of paths.

We now fix $\left(U_{1}, \cdots, U_{m}\right) \in E_{2}^{<}(m)$, consider some $\left(U_{1}^{\prime}, \cdots, U_{m}^{\prime}\right) \in E_{2}(m)$ and choose a path $\gamma$ joining them as above. We define

$$
\mu_{U_{1}^{\prime}, \cdots, U_{m}^{\prime}}=M_{\gamma}\left(\mu_{U_{1}, \cdots, U_{m}}\right) .
$$

For this to be well-defined, we need to establish the following. 
Lemma 4.3.2. Suppose $\gamma$ is a closed path in $E_{2}(m)$ with $\gamma(0)=\gamma(1)=\left(U_{1}, \cdots, U_{m}\right) \in$ $E_{2}^{<}(m)$. Then $M_{\gamma}\left(\mu_{U_{1}, \cdots, U_{m}}\right)=\mu_{U_{1}, \cdots, U_{m}}$.

Proof: This follows from the identification, up to quasi-isomorphism, of the stalks of the $\mathcal{E}_{n}^{\bullet}(A)$ in Proposition 4.2.9. That is, the stalks at $\mathbf{z}=\sum_{i=1}^{m} \lambda_{i} z_{i}, z_{i} \neq z_{j}$, are, up to quasiisomorphism (in particular, at the level of their cohomology), manifestly factorizable whether $z_{1} \prec \cdots<z_{m}$ or not. This means that the two morphisms of perverse sheaves, $M_{\gamma}\left(\mu_{U_{1}, \cdots, U_{m}}\right)$ and $\mu_{U_{1}, \cdots, U_{m}}$, induce the same maps on the cohomology of the stalks of the perverse sheaves in question. This, in its turn, implies that these two morphisms of perverse sheaves coincide.

Well-definedness being established, we easily conclude that:

Proposition 4.3.3. The isomorphisms (4.3.1) make $\mathcal{E}(A)=\left(\mathcal{E}_{n}^{\bullet}(A)\right)$ into a factorizable perverse sheaf on $\operatorname{Sym}(\mathbb{C})$ with values in $\mathcal{V}$.

B. Proof of Theorem 3.3.1. Proposition 4.3.3 defines a functor

$$
L: \operatorname{PB}(\mathcal{V}) \longrightarrow \operatorname{FPS}(\mathcal{V}), \quad A \mapsto L(A)=\left(L_{n}(A)\right)_{n \geqslant 0}, \quad L_{n}(A):=\mathcal{E}_{n}^{\bullet}(A)
$$

To prove that $L$ is an equivalence, we construct a functor in the opposite direction

$$
\Phi: \operatorname{FPS}(\mathcal{V}) \longrightarrow \operatorname{PB}(\mathcal{V}), \quad \mathcal{F}=\left(\mathcal{F}_{n}\right)_{n \geqslant 0} \mapsto \Phi(\mathcal{F})=\bigoplus_{n \geqslant 0} \Phi_{n}(\mathcal{F}), \quad \Phi_{n}(\mathcal{F}):=\Phi_{\text {tot }}\left(\mathcal{F}_{n}\right) .
$$

We recall (2.3.5) that $\Phi_{\text {tot }}\left(\mathcal{F}_{n}\right)$ is the stalk at 0 of the sheaf $R_{K_{n}}\left(\mathcal{F}_{n}\right)=\underline{H}_{K_{n}}^{0}(\mathcal{F})$, where $K_{n}=\operatorname{Sym}^{n}(\mathbb{R})$. As $\mathcal{F}$ is factorizable, we see that the stalk of $R_{K_{n}}\left(\mathcal{F}_{n}\right)$ at the stratum $K_{\alpha}$, $\alpha=\left(\alpha_{1}, \cdots, \alpha_{m}\right) \in \mathbf{2}^{n-1}$, is canonically identified with $\Phi_{\alpha_{1}}(\mathcal{F}) \otimes \cdots \otimes \Phi_{\alpha_{m}}(\mathcal{F})$. Consider the generalization maps from strata corresponding to $\alpha$ with $l(\alpha)=1$ to strata corresponding to $\alpha$ with $l(\alpha)=2$, for the sheaf structures of the $R_{K_{n}}\left(\mathcal{F}_{n}\right)$. These maps have the form $\Delta_{p, q}: \Phi_{n}(\mathcal{F}) \rightarrow \Phi_{p}(\mathcal{F}) \otimes \Phi_{q}(\mathcal{F}), p+q=n$. So they give a comultiplication $\Delta$ on $\Phi(\mathcal{F})$. This comultiplication is coassociative, as one can see by considering strata corresponding to $\alpha$ with $l(\alpha)=3$. It also satisfies the primitivity condition because $\mathcal{F}_{0}=\mathbf{1}$.

To define a multiplication on $\Phi(\mathcal{F})$ we use the Cousin resolution of $\mathcal{F}_{n}$ from Proposition 2.3.7. That is, we denote $j_{\alpha}: X_{\alpha}^{\mathfrak{I}} \rightarrow \operatorname{Sym}^{n}(\mathbb{C})$ the embedding of the imaginary stratum and put $\widetilde{\mathcal{E}}^{\alpha}\left(\mathcal{F}_{n}\right)=j_{\alpha}^{!} \mathcal{F}_{n}$, which is a single sheaf on $X_{\alpha}^{\mathfrak{I}}$. Then we put $\mathcal{E}^{\alpha}\left(\mathcal{F}_{n}\right)=j_{\alpha *} \widetilde{\mathcal{E}}^{\alpha}\left(\mathcal{F}_{n}\right)$, which is a single sheaf of $\operatorname{Sym}^{n}(\mathbb{C})$, and these sheaves, taken for all $\alpha \in 2^{n-1}$, form a complex quasi-isomorphic to $\mathcal{F}_{n}$.

Now, fix a point $\mathbf{z} \in X_{\alpha}^{\mathfrak{I}}$. We can write $\mathbf{z}=\sum \mathbf{z}_{i}$ where $\mathbf{z}_{i}$ is a divisor of degree $\alpha_{i}$ with all the points on the same horizontal line $\operatorname{Im}(z)=$ const. Therefore the pair $\left(X_{\alpha}^{I}\right.$ en, $\left.\operatorname{Sym}^{n}(\mathbb{C})\right)$ near $\mathbf{z}$ is homeomorphic to the product of the pairs $\left(\operatorname{Sym}^{\alpha_{i}}(\mathbb{R}), \operatorname{Sym}^{\alpha_{i}}(\mathbb{C})\right)$ near $\mathbf{z}_{i}$. Together with factorizability of $\mathcal{F}$ this implies that the stalk of $\widetilde{\mathcal{E}}^{\alpha}\left(\mathcal{F}_{n}\right)$ at $\mathbf{z}$ is the tensor product of the stalks of $\underline{H}_{K_{\alpha_{i}}}^{0}\left(\mathcal{F}_{\alpha_{i}}\right)$ at $\mathbf{z}_{i}$. This further implies that the stalk at 0 of the direct image is identified as

$$
\mathcal{E}^{\alpha}\left(\mathcal{F}_{n}\right)_{0}=\Phi_{\alpha_{1}}(\mathcal{F}) \otimes \cdots \otimes \Phi_{\alpha_{m}}(\mathcal{F})
$$


Let now $\delta$ be the differential in the Cousin complex of $\mathcal{F}_{n}$. As a graded sheaf, this complex is the sum of summands

$$
\bigoplus_{\alpha \in \mathbf{2}^{n-1}} \mathcal{E}^{\alpha}\left(\mathcal{F}_{n}\right)[l(\alpha)]
$$

Consider the action of the components of $\delta$ on the stalks of these summands at 0 . The components acting from the summands with $l(\alpha)=2$ to the summands with $l(\alpha)=1$ have the form $\mu_{p, q}: \Phi_{p}(\mathcal{F}) \otimes \Phi_{q}(\mathcal{F}) \rightarrow \Phi_{n}(\mathcal{F}), p+q=n$. So they define a multiplication $\mu$ in $\Phi(\mathcal{F})$. The condition $\delta^{2}=0$ implies the associativity of $\mu$. The compatibility of $\delta$ and $\mu$ follows from the fact that $\delta$ is a morphism of sheaves, i.e., commutes with the generalization maps, by the same analysis as in the proof of Proposition 4.2.2.

This defines the functor $\Phi$. If $\mathcal{F}$ is a factorizable perverse sheaf, then the above reasoning identifies the complex $\mathcal{E}^{\bullet}\left(\mathcal{F}_{n}\right)$ with $\mathcal{E}_{n}^{\bullet}(\Phi(A))$, and this shows that $L(\Phi(\mathcal{F})) \simeq \mathcal{F}$. If $A$ is a primitive bialgebra, then $\Phi(L(A)) \simeq A$ by Propositions 4.2.9 and 4.2.15. This shows that $L$ is an equivalence of categories.

Further, Proposition 4.2.9 implies properties (a) and (d) of the functor $L$ claimed in Theorem 3.3.1. Proposition 4.2.15 implies properties (b) and (c). Finally, to show the property (e) it is enough to notice that the Verdier duality interchanges stalks and costalks so the above identification of the multiplication and comultiplication in $A=\Phi(\mathcal{F})$ identifies comultiplication and multiplication in $\Phi(\mathbb{D} \mathcal{F})$, thus showing that $\Phi(\mathbb{D} \mathcal{F}) \simeq A^{*}$. Theorem 3.3.1 is proved.

\subsection{Perverse sheaves corresponding to shuffle algebras: proof of Theorem 3.3.3}

Here we prove Theorem 3.3.3, with the three paragraphs A,B,C below corresponding to parts (a), (b), (c) of the theorem.

A. Localization of the tensor algebra $T_{!}(V)$. Let $A=T_{!}(V)$, so $A_{n}=V^{\otimes n}$. We know that

$$
\left.\mathcal{E}_{n}^{\bullet}(A)\right|_{\operatorname{Sym}_{\neq}^{n}(\mathbb{C})}=\mathcal{L}_{\left(1^{n}\right)}(A)=\mathcal{L}_{n}(V)[n]
$$

is the local system of determinations of $V^{\otimes n}$, pu in degree $(-n)$. To show that $\mathcal{E}_{n}^{\bullet}(A) \simeq$ $j_{n !} \mathcal{L}_{n}(V)[n]$ it is enough to show that the stalk of $\mathcal{E}_{n}^{\bullet}(A)$ at any point $\mathbf{z} \in \operatorname{Sym}^{n}(\mathbb{C}) \backslash \operatorname{Sym}_{\neq}^{n}(\mathbb{C})$ is an exact complex. By Proposition 4.2.9, this stalk is the tensor product of the $B_{\lambda_{i}}(A)$, where $\mathbf{z}=\sum \lambda_{i} z_{i}$ with $z_{i}$ distinct. The condition $\mathbf{z} \in \operatorname{Sym}^{n}(\mathbb{C}) \backslash \operatorname{Sym}_{\neq}^{n}(\mathbb{C})$ means that some $\lambda_{i} \geqslant 2$. So our statement follows from the following.

Lemma 4.4.1. If $A=T_{!}(V)$, then for any $m \geqslant 2$ the complex $B_{m}(A)$ is exact.

For example, for $m=2$ the complex $B_{2}(A)=\left\{A_{1} \otimes A_{1} \stackrel{\mu}{\rightarrow} A_{2}\right\}$ is identified with $\{V \otimes V \stackrel{\text { Id }}{\rightarrow} V \otimes V\}$ and so is exact. 
Proof of the lemma: For any graded algebra $A$ we can write $B_{m}(A)$ as the graded object as follows:

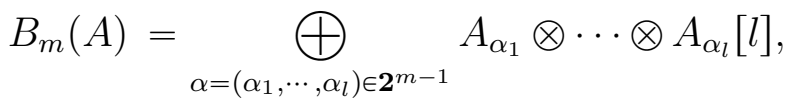

with the differential given by the multiplication in $A$. In our case $A_{\alpha_{i}}=V^{\otimes \alpha_{i}}$ and each component of the multiplication is the identity map. Therefore we have an isomorphism of complexes

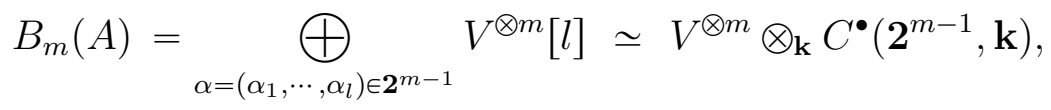

where

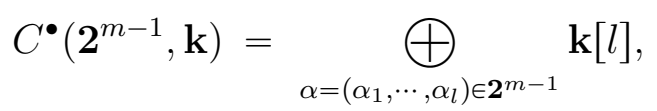

with the differential being the alternating sum of elementary contractions of ordered partitions. Now notice that $C^{\bullet}\left(\mathbf{2}^{m-1}, \mathbf{k}\right)$ is (up to shift) nothing but the augmented cellular chain complex of the $(n-2)$-dimensional simplex and so is exact.

B. Localization of the cotensor algebra $T_{*}(V)$. Let $A=T_{*}(V)$, so $A_{n}=V^{\otimes n}$ as before but the multiplication is the shuffe product while the comultiplication $\Delta_{p q}$ : $A_{p+q} \rightarrow A_{p} \otimes A_{q}$ is the identity. As before, $\left.\mathcal{E}_{n}^{\bullet}(A)\right|_{\operatorname{Sym}_{\neq}^{n}(\mathbb{C})}=\mathcal{L}_{n}(A)[n]$. To prove that $\mathcal{E}_{n}^{\bullet}(A) \simeq j_{*} \mathcal{L}_{n}(A)[n]$, it is enough to prove that the costalks $i_{\mathbf{z}}^{!} \mathcal{E}_{n}^{\bullet}(A)$ are exact for any $\mathbf{z} \in \operatorname{Sym}^{n}(\mathbb{C}) \backslash \operatorname{Sym}_{\neq}^{n}(\mathbb{C})$. This follows from Proposition 4.2.15 and from the statement dual to Lemma 4.4.1:

Lemma 4.4.2. Let $A=T_{*}(V)$. Then for any $m \geqslant 2$ the $m$ th cobar-complex $B_{m}^{\dagger}(A)$ is exact.

Proof: completely parallel to that of Lemma 4.4.1.

C. Localization of the Nichols algebra $T_{! *}(V)$. We notice the following:

(1) The canonical map $\alpha: T_{!}(V) \rightarrow T_{*}(V)$ is the unique morphism of algebras extending the identity on $T_{!}(V)_{1}=V=T_{*}(V)_{1}$.

(2) The canonical map $c_{n}: j_{n !} \mathcal{L}_{n}(V) \rightarrow j_{n *} \mathcal{L}_{n}(V)$ is the unique morphism of perverse sheaves which is the identity on $j_{n}^{*} j_{n !} \mathcal{L}_{n}(V)=\mathcal{L}_{n}(V)=j_{n}^{*} j_{n *} \mathcal{L}_{n}(V)$.

So Theorem 3.3.1 on equivalence between factorizable perverse sheaves and primitive bialgebras implies that $\alpha$ corresponds, under this equivalence, to the collection of the $c_{n}$. In other words, the functor $\Phi_{\text {tot }}$, see Theorem 3.3.1(b), takes $j_{n !} \mathcal{L}_{n}(V)$ as well as $j_{n *} \mathcal{L}_{n}(V)$ to $V^{\otimes n}$, and takes the map $c_{n}$ to $\alpha_{n}$, the $n$th component of $\alpha$.

Now, $\Phi_{\text {tot }}$ is an exact functor from $\operatorname{Perv}\left(\operatorname{Sym}^{n}(\mathbb{C}), S, \mathcal{V}\right)$ to $\mathcal{V}$ and so takes images to images. Therefore it takes $\left(j_{n}\right)_{! *} \mathcal{L}_{n}(V)$ to $T_{! *}(V)_{n}$, the $n$th component of the Nichols algebra. This finishes the proof of Theorem 3.3.3. 


\section{Examples: Granger-Maisonobe quivers and bialgebras (Cases $n=2,3$ )}

\subsection{Generalities: reducing translation invariance}

In this chapter we reivew the known elementary classification of perverse sheaves on $\operatorname{Sym}^{n}(\mathbb{C})$ for $n=2,3$, smooth with respect to the diagonal stratification $S$. We also show how a primitive braided bialgebra $A$ gives, in an elementary way, a perverse sheaf (denoted by $L_{n}(A)$ in Theorem 3.3.1) in terms of these descriptions.

Let $\mathcal{V}$ be a k-linear braided monoidal abelian category with $\otimes$ biexact.

As before, let $\operatorname{Sym}_{0}^{n}(\mathbb{C}) \subset \operatorname{Sym}_{n}(\mathbb{C})$ be the subspace of monic polynomials with zero subprincipal part, see (2.1.2) ff. Let $S^{0}$ be the stratification induced by $S$ on $\operatorname{Sym}_{0}^{n}(\mathbb{C})$. Since $S$ is translation invariant, we have an identification

$$
\operatorname{Perv}\left(\operatorname{Sym}^{n}(\mathbb{C}), S, \mathcal{V}\right) \simeq \operatorname{Perv}\left(\operatorname{Sym}_{0}^{n}(\mathbb{C}), S^{0}, \mathcal{V}\right) .
$$

Note, however, that this identification involves a shift of degree: perverse sheaves on $\left(\operatorname{Sym}^{n}(\mathbb{C}), S\right)$ are situated, as complexes, in degrees $[-n,-1]$, while perverse sheaves on $\left.\operatorname{Sym}_{0}^{n}(\mathbb{C}), S^{0}\right)$ are situated in degrees $[-n+1,0]$. In particular, it does affect the concept of factorizable perverse sheaves because of the Koszul sign rule (3.2.3) involved in the braiding of shifted objects.

In this chapter we will work only with $\operatorname{Sym}_{0}^{n}(\mathbb{C})$, but keep the same notation that we used for various strata in $\operatorname{Sym}^{n}(\mathbb{C})$, assuming that they are intersected with $\operatorname{Sym}_{0}^{n}(\mathbb{C})$.

\subsection{Case $n=2$ : the $(\Phi, \Psi)$ description.}

Note that $\operatorname{Sym}_{0}^{2}(\mathbb{C})=\mathbb{C}$, with the complex strata $X_{(2)}^{\mathbb{C}}$ and $X_{1,1}^{\mathbb{C}}$ being $\{0\}$ and $\mathbb{C}-\{0\}$. Thus $\operatorname{Perv}\left(\operatorname{Sym}_{0}^{2}(\mathbb{C}), S^{0}, \mathcal{V}\right)$ is identified with the category $\operatorname{Perv}(\mathbb{C}, 0, \mathcal{V})$ studied in Example 1.1.5 (for the case of $\mathcal{V}=$ Vect $_{\mathbf{k}}$, which generalizes verbatim to arbitrary $\mathcal{V}$ ).

In other words, $\operatorname{Perv}\left(\operatorname{Sym}_{0}^{2}(\mathbb{C}), S^{0}, \mathcal{V}\right)$ is equivalent to the category of diagrams formed by two objects and two morphisms of $\mathcal{V}$

$$
E_{2}=\Phi \underset{u}{\stackrel{v}{\rightleftarrows}} \Psi=E_{1,1}
$$

such that the morphisms $T_{\Psi}=\mathrm{Id}_{\Psi}-v u$ and $T_{\Phi}=\mathrm{Id}_{\Phi}-u v$ are invertible.

Let $A$ be a primitive bialgebra in $\mathcal{V}$. We associate to it the diagram

$$
\Phi=A_{2} \underset{u=\mu_{1,1}}{\stackrel{v=\Delta_{1,1}}{\rightleftarrows}} A_{1} \otimes A_{1}=\Psi
$$

In the calculations below and in $§ 5.3$ later we will assume that we have a fully faithful embedding of $\mathcal{V}$, as a monoidal (but not as braided monoidal) category into Vect $_{\mathbf{k}}$, in other words, that objects $V$ of $\mathcal{V}$ have consist of elements, or vectors $x \in V$, so that the tensor 
product of two objects $V \otimes W$ consists of sums of formal tensors $x_{i} \otimes y_{i}$. This assumption is for notational simplification only: it is straightforward (but more lengthy) to restate our arguments entirely in terms of diagrams involving various tensor products in $\mathcal{V}$.

Then for $a \otimes b \in A_{1} \otimes A_{1}$ we get, using the assumption that $A_{1}$ consists of primitive elements:

$$
\begin{gathered}
v u(a \otimes b)=\Delta_{1,1}(a b)=(\Delta(a) \cdot \Delta(b))_{1,1}= \\
=((a \otimes 1+1 \otimes a)(b \otimes 1+1 \otimes b))_{1,1}=(a \otimes 1)(1 \otimes b)+(1 \otimes a)(b \otimes 1)= \\
=a \otimes b+R_{A_{1}, A_{1}}(a \otimes b),
\end{gathered}
$$

the last equality coming from the fact that the multiplication in $A \otimes A$ is defined using $R$. Therefore

$$
T_{\Psi}=\mathrm{Id}-v u=-R_{A_{1}, A_{1}}: A_{1} \otimes A_{1} \longrightarrow A_{1} \otimes A_{1}
$$

is the negative of braiding operator and it is invertible by axioms of braided monoidal categories. This minus sign is an instance of the twist of the braid group action by the sign character in Remark 3.3.2.

Further, the invertibility of

$$
T_{\Phi}=\mathrm{Id}-\mu_{1,1} \circ \Delta_{1,1}: A_{2} \longrightarrow A_{2}
$$

follows from that of $T_{\Psi}$ by (1.1.6). Note that $T_{\Phi}=\sigma_{2}$ is just the antipode of $A$ acting on $A_{2}$.

\subsection{Case $\mathrm{n}=3$ : the Granger-Maisonobe quiver.}

A. Imaginary strata for $n=3$. We have that $\operatorname{Sym}_{0}^{3}(\mathbb{C})=\mathbb{C}^{2}$ is the space of cubic polynomials of the form $x^{3}+a x+b$. The open stratum $X_{(1,1,1)}^{\mathbb{C}}$ consists of polynomials with distinct roots, i.e., with non-vanishing discriminant. It is therefore the complement of the complex semi-cubic parabola

$$
Z=\left\{(a, b) \in \mathbb{C}^{2} \mid 4 a^{3}+27 b^{2}=0\right\} .
$$

The other two strata $X_{(2,1)}^{\mathbb{C}}$ and $X_{(3)}^{\mathbb{C}}$ are equal to $Z \backslash\{0\}$ (the smooth locus of $Z$ ) and $\{0\}$.

The category of perverse sheaves on $\mathbb{C}^{2}$ smooth with respect to a semi-cubic parabola was described in the papers of Granger-Maisonobe [26] and MacPherson-Vilonen [46] (the latter authors considering the more general case of a curve of the form $a^{m}=b^{n}$ ).

We want to emphasize that our method (in the main body of the paper) is a direct generalization of the Granger-Maisonobe approach. In particular, the cuts ("coupures") $H$ and $M_{\mathbb{R}}$ of [26] (whose idea is attributed by the authors to Kyoji Saito) are precisely the unions of imaginary strata $X_{(2,1)}^{\mathfrak{I}} \cup X_{(1,2)}^{\mathfrak{I}}$ and $X_{(3)}^{\mathfrak{I}}=\operatorname{Sym}_{0}^{3}(\mathbb{R})$ in our sense. So we can say, together with the authors of [26], that K. Saito "est a l'origine de ce travail". 
More precisely, for $n=3$ we have 4 imaginary strata:

$$
X_{(3)}^{\mathfrak{I}}=\operatorname{Sym}_{0}^{3}(\mathbb{R}), X_{(1,2)}^{\mathfrak{I}}, X_{(2,1)}^{\mathfrak{I}}, X_{(1,1,1)}^{\mathfrak{I}}
$$

whose real dimensions are equal to $2,3,3,4$ respectively. Thus $X_{(1,1,1)}^{\mathfrak{I}} \subset \operatorname{Sym}_{0}^{3}(\mathbb{C})=\mathbb{C}^{2}$ is open, while $X_{(1,2)}^{\mathfrak{I}}$ and $X_{(2,1)}^{\mathfrak{I}}$ are locally closed 3-dimensional subanalytic hypersurfaces with boundary. The boundary of each of them is formed by $Z \backslash\{0\}$ (this boundary actually lies in the stratum). Further, each of them contains in their closure (but does not actually contain) the stratum $X_{(3)}^{\mathfrak{I}}$.

For further visualization it is instructive to take, as proposed in [26], the intersection of the entire picture with the unit sphere $S^{3}=\left\{|a|^{2}+\left|b^{2}\right|=1\right\} \subset \mathbb{C}^{2}$. The intersection $T=Z \cap S^{3}$ is, as well known, a trefoil knot in $S^{3}$. The intersection with $S^{3}$ of $X_{(3)}^{\mathfrak{I}}$, i.e., of the interior of the real semi-cubic parabola, see Fig. 3, is the same as the intersection of this interior with the unit circle in $\mathbb{R}^{2}$. So it is an interval (arc) in $S^{3}$ beginning and ending on $T$ and not meeting $T$ at any other points, i.e., a chord $C$ of $T$. The two points of $C \cap T$, denote them $a$ and $b$, subdivide $T$ into two halves $T_{+}$and $T_{-}$.

The intersections $X_{(1,2)}^{\mathfrak{I}} \cap S^{3}$ and $X_{(2,1)}^{\mathfrak{I}} \cap S^{3}$ are 2-dimensional locally closed surfaces in $S^{3}$, with boundary. They have $T_{+}$resp. $T_{-}$as the boundary and approach (but do not actually contain) the chord $C$. All parts of this picture are identified with (the intersection with $S^{3}$ of) various fine cells. This is shown symbolically in Fig. 5, where $T$ is represented as a circle but knottedness is not depicted.

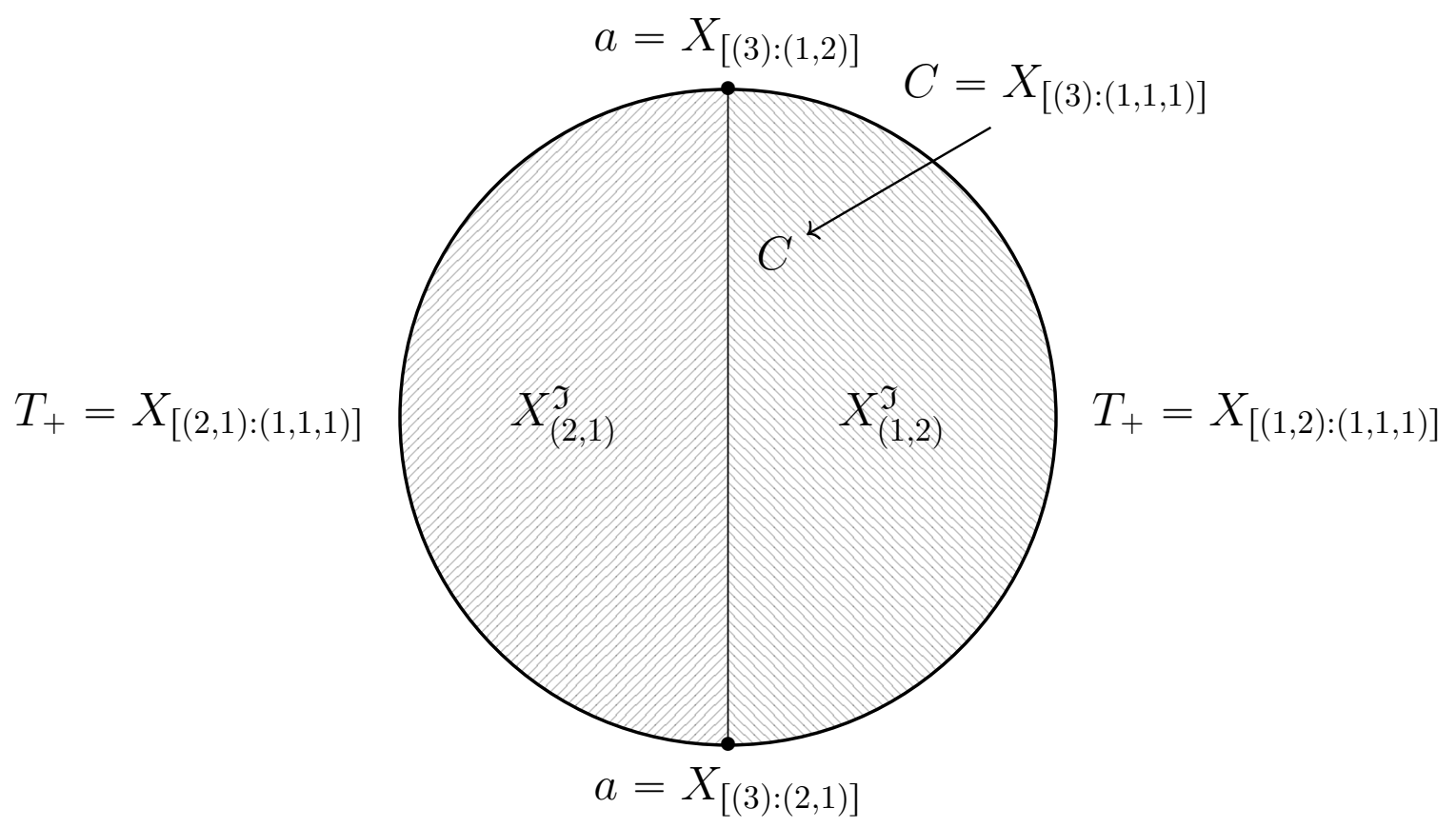

Figure 5: Imaginary strata and fine cells intersected with $S^{3}$. Knottedness not depicted. 
B. The Granger-Maisonobe quiver. The description of $\operatorname{Perv}\left(\operatorname{Sym}_{0}^{3}(\mathbb{C}), S^{0}\right.$, Vect $)$ was given in [26] is in terms of diagrams of 4 vector spaces denoted there by $E, F_{1}, F_{2}, G$. Our method being a direct generalization of the method of the Granger-Maisonobe approach, their spaces have transparent interpretation in our general terms. We reproduce this type of diagram below, keeping their notation on the right and adding our more systematic notation on the left:

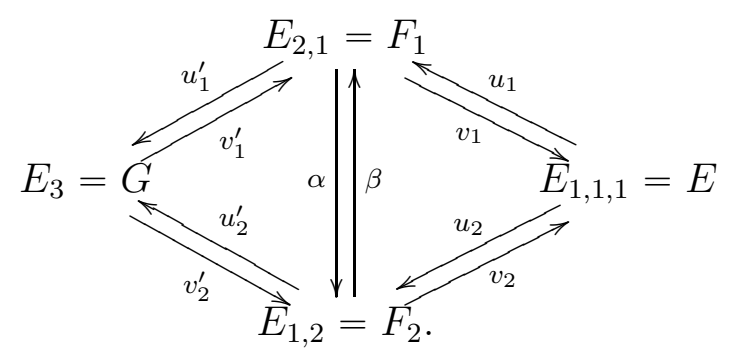

We note that the arguments of [26] generalize verbatim to perverse sheaves with values in any abelian category, so we have:

Theorem 5.3.2 ([26]). Let $\mathcal{V}$ be any $\mathbf{k}$-linear abelian category (not necessarily monoidal). $\operatorname{Perv}\left(\operatorname{Sym}_{0}^{3}(\mathbb{C}), S^{0}, \mathcal{V}\right)$ is equivalent to the category of diagrams (5.3.1) formed by objects and morphisms of $\mathcal{V}$ and satisfying the following relations:

(1) $M_{1}=v_{1} u_{1}+\operatorname{Id}$ and $M_{2}=v_{2} u_{2}+\operatorname{Id}$ are isomorphisms.

(2) $u_{1}^{\prime} u_{1}+u_{2}^{\prime} u_{2}=0$ and $v_{1} v_{2}^{\prime}+v_{2} v_{2}^{\prime}=0$.

(3) $v_{2}^{\prime} u_{1}^{\prime}=-\alpha+u_{2} v_{1}$ and $v_{1}^{\prime} u_{2}^{\prime}=-\beta+u_{1} v_{2}$.

(4) $\alpha$ and $\beta$ are isomorphisms.

(5) $v_{1}^{\prime} u_{1}^{\prime}=\mathrm{Id}+\beta u_{2} M_{1}^{-1} v_{1}$ and $v_{2}^{\prime} u_{2}^{\prime}=\mathrm{Id}+\alpha u_{1} M_{2}^{-1} v_{2}$.

(6) $v_{2} \alpha=M_{1} M_{2} v_{1}$ and $v_{1} \beta=M_{2} M_{1} v_{2}$.

Let us analyze this result in a series of remarks.

Remarks 5.3.3. (a) The relations (3) imply that $\alpha$ and $\beta$ are expressed through the rest of the generators.

(b) The relations (2) mean that the remaining generators form, up to replacing some arrows with their negatives, a double representation of $\mathbf{2}^{3-1}$ in $\mathcal{V}$, i.e., we get a commutative square

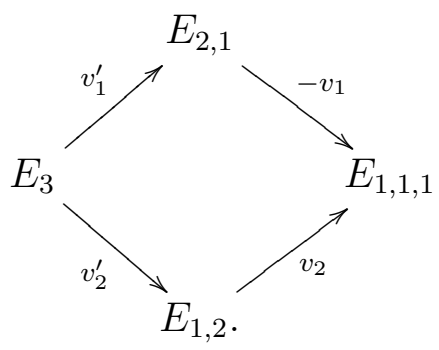


and another commutative square obtained from the " $u$-part" of the diagram.

(c) Further, let $\mathcal{F} \in \operatorname{Perv}\left(\operatorname{Sym}_{0}^{3}(\mathbb{C}), S^{0}, \mathcal{V}\right)$. Then the commutative square in (b) obtained from the diagram (5.3.1) corresponding to $\mathcal{F}$, precisely describes the constructible sheaf $R_{K^{0}}(\mathcal{F})$ on the real skeleton $K^{0}=\operatorname{Sym}_{0}^{3}(\mathbb{R})$. This means that $E_{\alpha}$ is the stalk of $R_{K^{0}}(\mathcal{F})$ at the stratum $K_{\alpha}^{0}=K^{0} \cap K_{\alpha}, \alpha \in 2^{3-1}$, while the $v_{i}, v_{i}^{\prime}$ are the generalization maps, see [26] $\S I I .4$.

(d) From this point of view, the rest of relations in Theorem 5.3.2 can be viewed as giving a precise meaning to the concept of a bi-sheaf on $K^{0}$, by specifying the compatibility conditions between the sheaf structure ( $v$-part) and the cosheaf structure (u-part).

\section{Granger-Maisonobe relations from a braided bialgebra.}

Theorem 5.3.4. Let $A$ be a primitive bialgebra in a braided monoidal category $(\mathcal{V}, \otimes, R)$. Let us associate to it the diagram (5.3.1) by:

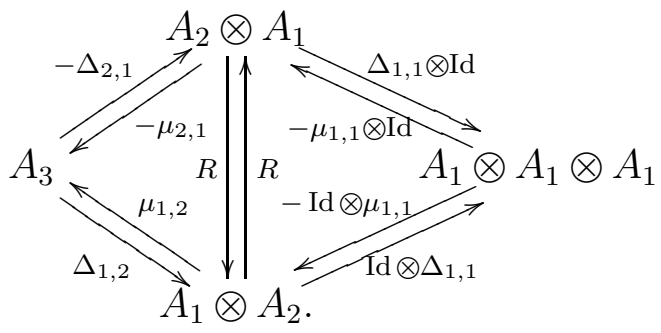

That is, up to signs, the maps $u_{i}, u_{i}^{\prime}$ are given by the multiplication, $v_{i}, v_{i}^{\prime}$ by the comultiplication (so they form the bibar-cube $\mathbb{B}_{3}(A)$ in $\mathcal{V}$ ) while $\alpha=R_{A_{2}, A_{1}}$ and $\beta=R_{A_{1}, A_{2}}$ are the braiding isomorphisms. Then this diagram satisfies the relations of Theorem 5.3.2.

Proof: The condition (1) is fulfilled, in virtue of (5.2.1), with

$$
M_{1}=-R_{A_{1}, A_{1}} \otimes \mathrm{Id}, \quad M_{2}=-\mathrm{Id} \otimes R_{A_{1}, A_{1}} .
$$

As in the case $n=2$, these minus signs are an instance of the braid group action being twisted by the sign character (Remark 3.3.2).

The conditions (4) are obvious. The relations (2) follow from associativity and coassociativity. Let us check the remaining relations directly.

Relations (5). Let us check the relation

$$
v_{1}^{\prime} u_{1}^{\prime}=\mathrm{Id}+\beta u_{2} M_{1}^{-1} v_{1}: F_{1} \longrightarrow F_{1},
$$

that is,

$$
\left(-\Delta_{2,1}\right)\left(-\mu_{2,1}\right)=\mathrm{Id}+R_{A_{1}, A_{2}}\left(-\mathrm{Id} \otimes \mu_{1,1}\right)\left(-R_{A_{1}, A_{1}}^{-1} \otimes \mathrm{Id}\right)\left(\Delta_{1,1} \otimes \mathrm{Id}\right): A_{2} \otimes A_{1} \longrightarrow A_{2} \otimes A_{1} .
$$


Let $x=a_{2} \otimes a \in A_{2} \otimes A_{1}=F_{1}$. As traditional in the theory of Hopf algebras ("Sweedler notation"), we write symbolically

$$
\Delta\left(a_{2}\right)_{11}=b \otimes c
$$

meaning by $b \otimes c$ a sum of the form $\sum b_{i} \otimes c_{i}$. Let us also write, in the same way,

$$
R(c \otimes a)=c^{\prime} \otimes a^{\prime}, \quad R=R_{A_{1}, A_{1}} .
$$

Then

$$
\begin{gathered}
v_{1}^{\prime} u_{1}^{\prime}(x)=\left[\Delta\left(a_{2} a\right)\right]_{2,1}=\left[\left(a_{2} \otimes 1+\Delta\left(a_{2}\right)_{11}+1 \otimes a_{2}\right)(a \otimes 1+1 \otimes a)\right]_{2,1}= \\
=a_{2} \otimes a+\Delta\left(a_{2}\right)_{11}(a \otimes 1)=a_{2} \otimes a+b a^{\prime} \otimes c^{\prime}
\end{gathered}
$$

i.e.,

$$
\left(v_{1}^{\prime} u_{1}^{\prime}-\mathrm{Id}\right)(x)=b a^{\prime} \otimes c^{\prime}
$$

On the other hand

$$
\beta u_{2} M_{1}^{-1} v_{1}(x)=\beta u_{2} M_{1}^{-1}(b \otimes c \otimes a)=R_{A_{1}, A_{2}}(\tilde{c} \otimes \tilde{b} a)
$$

where

$$
R^{-1}(b \otimes c)=\tilde{c} \otimes \tilde{b}
$$

We have to show that

$$
b a^{\prime} \otimes c^{\prime}=R_{A_{1}, A_{2}}(\tilde{c} \otimes \tilde{b} a) .
$$

We have a commutative diagram (naturality of the braiding)

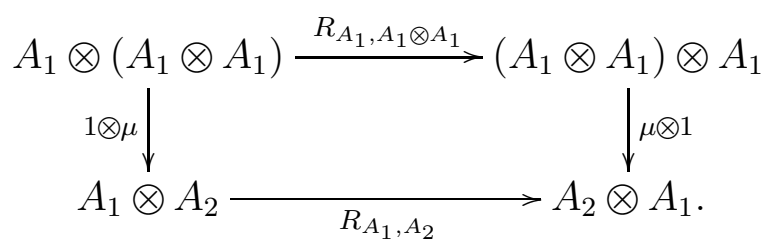

On the other hand, $R_{A_{1}, A_{1} \otimes A_{1}}$ equals the composition

$$
A_{1} \otimes A_{1} \otimes A_{1} \stackrel{R_{12}}{\longrightarrow} A_{1} \otimes A_{1} \otimes A_{1} \stackrel{R_{23}}{\longrightarrow} A_{1} \otimes A_{1} \otimes A_{1},
$$

whence

$$
R_{A_{1} \otimes A_{1}, A_{1}} R_{12}^{-1}=R_{23}
$$

It follows:

$$
R_{A_{1}, A_{2}}(1 \otimes \mu) R_{12}^{-1}=(\mu \otimes 1) R_{A_{1}, A_{1} \otimes A_{1}} R_{12}^{-1}=(\mu \otimes 1) R_{23},
$$

thus

$$
R_{A_{1}, A_{2}}(1 \otimes \mu) R_{12}^{-1}(b \otimes c \otimes a)=(\mu \otimes 1) R_{23}(b \otimes c \otimes a),
$$

and this is the sought for relation (5.3.5). The second relation in (5) is proved similarly. 
Relations (3). Let us check that

$$
v_{2}^{\prime} u_{1}^{\prime}=-\alpha+u_{2} v_{1}: F_{1} \longrightarrow F_{2},
$$

i.e.,

$$
\Delta_{1,2}\left(-\mu_{2,1}\right)=-R_{A_{2}, A_{1}}+\left(-\mathrm{Id} \otimes \mu_{1,1}\right)\left(\Delta_{1,1} \otimes \mathrm{Id}\right): A_{2} \otimes A_{1} \longrightarrow A_{1} \otimes A_{2} .
$$

Dropping the minus signs, we write this as

$$
\Delta_{1,2} \mu_{2,1}=R_{A_{2}, A_{1}}+\left(\mathrm{Id} \otimes \mu_{1,1}\right)\left(\Delta_{1,1} \otimes \mathrm{Id}\right) .
$$

As before, let $a_{2} \otimes a \in A_{2} \otimes A_{1}$ and let $\Delta\left(a_{2}\right)_{11}=b \otimes c$. Then

$$
\begin{aligned}
\Delta_{1,2} \mu_{2,1}\left(a_{2} \otimes a\right)= & {\left[\left(a_{2} \otimes 1+b \otimes c+1 \otimes a_{2}\right)(a \otimes 1+1 \otimes a)\right]_{12}=} \\
& =\left(1 \otimes a_{2}\right)(a \otimes 1)+b \otimes c a .
\end{aligned}
$$

But, by definition of the product in $A \otimes A$,

$$
\left(1 \otimes a_{2}\right)(a \otimes 1)=(\mu \otimes \mu)\left(1 \otimes R_{A_{2}, A_{1}}\left(a_{2} \otimes a\right) \otimes 1\right)=R_{A_{2}, A_{1}}\left(a_{2} \otimes a\right) .
$$

So the LHS of (5.3.8), applied to $a_{2} \otimes a$, is equal to

$$
R_{A_{2}, A_{1}}\left(a_{2} \otimes a\right)+b \otimes c a,
$$

with the first summand in (5.3.9) being the same as the value, at $a_{2} \otimes a$, of the first summand in the RHS of (5.3.8). Now, the second summand in the RHS of (5.3.8), applied to $a_{2} \otimes a$, is

$$
\left(\operatorname{Id} \otimes \mu_{1,1}\right)(b \otimes c \otimes a)=b \otimes c a,
$$

so it coincides with the second summand of (5.3.9) as well, thus proving (5.3.8) and (5.3.7). The second relation in (3) is proved similarly.

Relations (6): Let us check the identity

$$
v_{2} \alpha=M_{1} M_{2} v_{1}: F_{1} \longrightarrow E,
$$

i.e.,

$$
\left(\mathrm{Id} \otimes \Delta_{1,1}\right) R_{A_{2}, A_{1}}=\left(-\mathrm{Id} \otimes R_{A_{1}, A_{1}}\right)\left(-R_{A_{1}, A_{1}} \otimes \mathrm{Id}\right)\left(\Delta_{1,1} \otimes \mathrm{Id}\right): A_{2} \otimes A_{1} \longrightarrow A_{1} \otimes A_{1} \otimes A_{1} .
$$

This follows from the commutative diagram (naturality of the braiding)

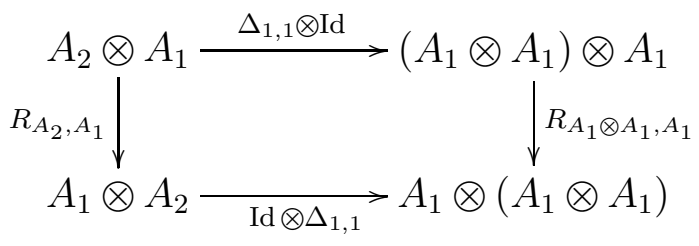

and the fact that

$$
R_{A_{1} \otimes A_{1}, A_{1}}=\left(\mathrm{Id} \otimes R_{A_{1}, A_{1}}\right)\left(R_{A_{1}, A_{1}} \otimes \mathrm{Id}\right) .
$$

The second relation in (6) is proved similarly. 


\section{A Shuffle complexes}

(A.1) The permutohedron and its faces. Let $n \geqslant 0$. By $\Sigma_{n}$ we denote the symmetric group Aut $\{1, \cdots, n\}$. The $n$th permutohedron is the convex polytope in $\mathbb{R}^{n}$ defined as

$$
P_{n}=\operatorname{Conv}\left\{[s], s \in \Sigma_{n}\right\}, \quad[s]:=(s(1), \cdots, s(n)) \in \mathbb{R}^{n} .
$$

The following is well known.

Proposition A.1. (a) $P_{n}$ is a convex polytope of dimension $n-1$ with $n$ ! vertices $[s], s \in \Sigma_{n}$.

(b) Faces of $P_{n}$ (of all dimensions) are labelled by sequences $\left(I_{1}, \cdots, I_{m}\right)$ of subsets $I_{\nu} \subset\{1, \cdots, n\}$ which form a disjoint decomposition of $\{1, \cdots, n\}$. The face $\left[I_{1}, \cdots, I_{m}\right]$ corresponding to $\left(I_{1}, \cdots, I_{m}\right)$ has vertices $[s]$ for all permutations s obtained by ordering elements in the $I_{\nu}$ in all possible ways. Thus

$$
\left[I_{1}, \cdots, I_{m}\right] \simeq P_{\left|I_{1}\right|} \times \cdots \times P_{\left|I_{m}\right|}
$$

is a product of permutohedra and has dimension $n-m$.

(A.2) The shuffle polytope and the shuffle complex. Let $\left(d_{1}, \cdots, d_{m}\right)$ be a sequence of non-negative integers and $d=d_{1}+\cdots+d_{m}$. Recall that a $\left(d_{1}, \cdots, d_{m}\right)$-shuffle is a permutation $s \in \Sigma_{d}$ whiose restriction onto every interval

$$
d_{1}+\cdots+d_{j}, d_{1}+\cdots+d_{j}+1, \cdots, d_{1}+\cdots+d_{j+1}-1, \quad j=1, \cdots, m-1,
$$

is monotone (order preserving). The set of $\left(d_{1}, \cdots, d_{m}\right)$-shuffles will be denoted $\amalg_{d_{1}, \cdots, d_{m}} \subset$ $\Sigma_{d}$.

Example A.3. In examples it is convenient to label integers from the intervals (A.2) by numbered letters such as

$$
a_{1}, \cdots, a_{d_{1}}, b_{1}, \cdots, b_{d_{2}}, c_{1}, \cdots, c_{d_{3}} \text {, etc. }
$$

Then a shuffle is a just reordering of these letters which preserves the order of $a$ 's, the order of $b$ 's etc. For example, $a_{1} b_{1} a_{2} b_{2}$ and $b_{1} b_{2} a_{1} a_{2}$ are $(2,2)$-shuffles.

Definition A.4. The shuffle polytope $N_{d_{1}, \cdots, d_{m}} \subset \mathbb{R}^{d}$ is the convex hull of the points [s], $s \in \amalg_{d_{1}, \cdots, d_{m}}$.

This $N_{d_{1}, \cdots, d_{m}} \subset P_{d}$, see Fig. 6 for an illustration. 


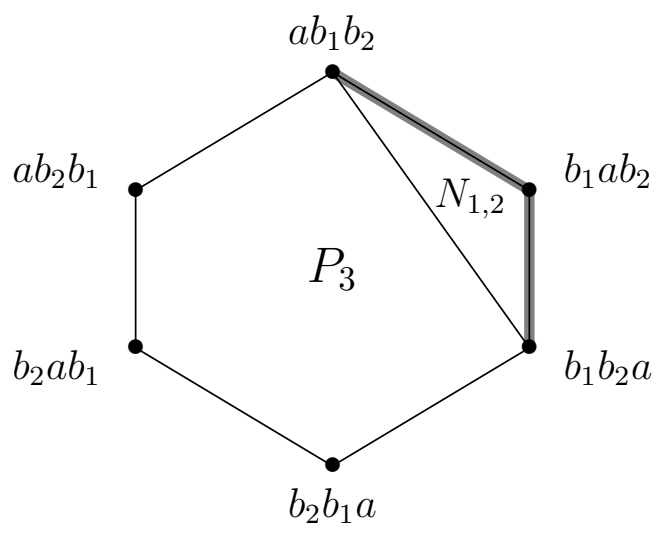

Figure 6: The hexagon $P_{3}$ and the triangle $N_{1,2}$.

Remark A.5. As pointed out in [23], Ch. 12, §2, see also [37], the 2-index shuffle polytope $N_{d_{1}, d_{2}}$ is the same as the resultohedron, i. e., the Newton polytope of the resultant $R\left(f_{1}, f_{2}\right)$ of two indeterminate polynomials of degrees $d_{1}, d_{2}$ :

$$
f_{1}(x)=u_{0} x^{d_{1}}+u_{1} x^{d_{1}-1}+\cdots+u_{d_{1}}, \quad f_{2}(x)=v_{0} x^{d_{2}}+v_{1} x^{d_{2}-1}+\cdots+v_{d_{2}} .
$$

An arbitrary $N_{d_{1}, \cdots, d_{m}}$ can also be interpreted in a similar way. For this we introduce $m$ indeterminate polynomials $f_{1}(x), \cdots, f_{m}(x)$ of degrees $d_{1}, \cdots, d_{m}$ and form the expression

$$
\mathcal{R}\left(f_{1}, \cdots, f_{m}\right)=\prod_{i<j} R\left(f_{i}, f_{j}\right)
$$

Then $N_{d_{1}, \cdots, d_{m}}$ can be identified with the Newton polytope of $\mathcal{R}$, i.e., with the Minkowski sum of the resultohedra $N_{d_{i}, d_{j}}$. We do not prove this fact here since we will not need it.

Definition A.6. The shuffle complex $\amalg \mathrm{D}_{d_{1}, \cdots, d_{m}}$ is the union of all the faces of $N_{d_{1}, \cdots, d_{m}} \subset$ $P_{d}$, which are also faces of $P_{d}$.

Examples A.7. (a) If $\left(d_{1}, \cdots, d_{m}\right)=(1, \cdots, 1)$, then

$$
N_{1, \cdots, 1}=\amalg \mathrm{D}_{1, \cdots, 1}=P_{m} .
$$

(b) $\amalg D_{1,2}$ is the union of the two edges of the hexagon $P_{3}$ represented by thick lines in Fig. 6.

(c) $\amalg \mathrm{D}_{2,2}$ is the complex in Fig. 7 . 


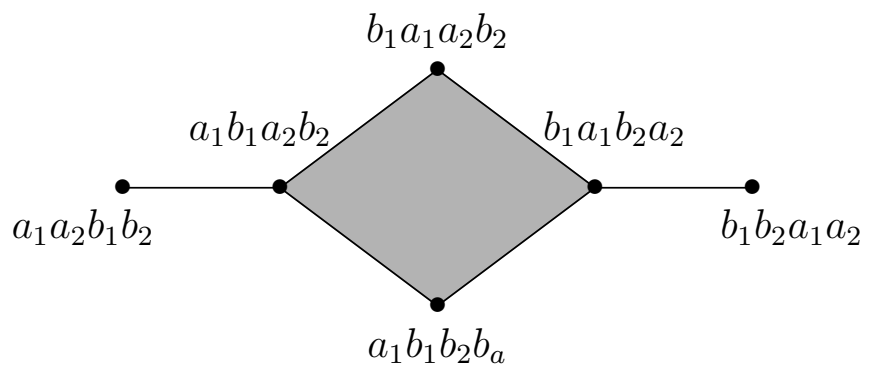

Figure 7: The complex $\amalg \mathrm{D}_{2,2}$.

Proposition A.8. Each $\amalg \mathrm{D}_{d_{1}, \cdots, d_{m}}$ is contractible.

The proof will be given in the next $\S($ A.3).

(A.3) Relation to the triangulated product of simplices. . For any $p \geqslant 0$ we consider the standard coordinate space $\mathbb{R}^{p+1}$ with basis $e_{0}, \cdots, e_{p}$. Let $\Delta^{p}=\operatorname{Conv}\left\{e_{0}, \cdots . e_{p}\right\} \subset$ $\mathbb{R}^{p+1}$ be the standard $p$-dimensional simplex witn vertices $e_{0}, \cdots, e_{p}$. It is convenient to identify the set of vertices of $\Delta^{p}$ with the standard ordinal $[p]=\{0,1, \cdots, p\}$.

Given $d_{1}, \cdots, d_{m}$ as before, we consider the product $\Pi=\Delta^{d_{1}} \times \cdots \times \Delta^{d_{m}}$. It is a convex polytope of dimension $d=d_{1}+\cdots+d_{m}$. We denote its vertices by $e_{\sigma}$ where $\sigma$ runs in the set $\left[d_{1}\right] \times \cdots \times\left[d_{m}\right]$ which we consider with the partial order given by the product of the standard orders on the $\left[d_{j}\right]$. We denote by $T$ the standard triangulation of $\Pi$ well known in the theory of simplicial sets, see, e.g., [24] §I.1.5. By definition, simplices of $T$ (of any dimension $m$ ) have the form

$$
\Delta_{\tau}=\operatorname{Conv}\left\{e_{\tau_{0}}, e_{\tau_{1}}, \cdots, e_{\tau_{m}}\right\}, \quad \tau=\left(\tau_{0}<\cdots<\tau_{m}\right), \quad \tau_{i} \in \prod_{j=1}^{m}\left[d_{j}\right],
$$

i.e., they are parametrized by chains in the poset $\prod_{j=1}^{m}\left[d_{j}\right]$. Proposition A.8 follows from the next more precise fact.

Proposition A.9. $\amalg \mathrm{D}_{d_{1}, \cdots, d_{m}}$ is identified with $T^{\vee}$, the polyhedral complex Poincaré dual to $T$.

Explicitly, to construct $T^{\vee}$, we take one vertex for each maximal simplex of $T$, these vertices being joined by an edge when the simplices are adjacent and so on. That is, cells of $T^{\vee}$ correspond to those simplices of $T$ that lie inside $\Pi$ (i.e., do not lie on its boundary), with the opposite order of inclusion.

Proof of Proposition A.9: Note first that maximal chains $\tau$ in $\prod\left[d_{j}\right]$ (with $m=d$ ) are in bijection with $\amalg_{d_{1}, \cdots, d_{m}}$. Indeed, such chains can be seen as "lattice paths" or "taxicab paths" $\mathfrak{p}$ in the $m$-dimensional parallelotope $\prod_{j=1}^{m}\left[0, d_{j}\right]$, going from the minimal vertex $(0, \ldots, 0)$ to the maximal one $\left(d_{1}, \cdots d_{m}\right)$. This is because, $\tau$ being maximal, each $\tau_{\nu+1}$ is obtained from $\tau_{\nu}$ by going exactly one step in one of the $l$ directions. See the left part of Fig. 8 for $m=2$. 
$b_{1} a_{1} a_{2} a_{3} b_{2} a_{4} a_{5} b_{3}$

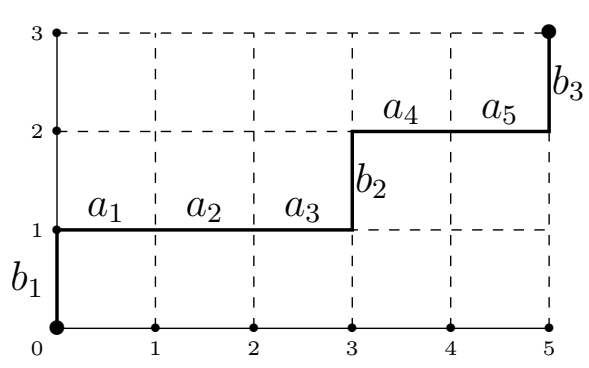

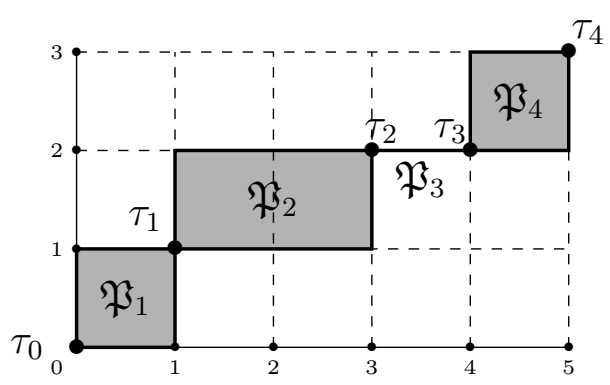

Figure 8: A taxicab path $\mathfrak{p}$ representing a shuffle and a block path $\mathfrak{P}$.

Further, consider simplices $\Delta_{\tau}$ corresponding to arbitrary chains $\tau=\left(\tau_{0}<\cdots<\tau_{m}\right)$. The condition for $\Delta_{\tau}$ to lie inside $\Pi$ is that $\tau_{0}$ is the minimal element $(0, \cdots, 0)$ of $\prod\left[d_{j}\right]$ and $\tau_{m}$ is the maximal element $\left(d_{1}, \cdots, d_{m}\right)$.

Notice that such $\tau$ are in bijection with block paths in the parallelotope $\prod_{j=1}^{m}\left[0, d_{j}\right]$, i.e., sequences $\mathfrak{P}=\left(\mathfrak{P}_{1}, \cdots, \mathfrak{P}_{m}\right)$ of lattice parallelotopes (possibly degenerate, i.e., of smaller dimension) inside $\prod_{j=1}^{m}\left[0, d_{j}\right]$ with the following properties:

(1) The minimal (with respect to the product partial order on $\mathbb{R}^{m}$ ) vertex of $\mathfrak{P}_{1}$ is $(0, \cdots, 0)$.

(2) For $i=1, \cdots, m-1$, the minimal vertex of $\mathfrak{P}_{i+1}$ coincides with the maximal vertex of $\mathfrak{P}_{i}$.

(3) The maximal vertex of $\mathfrak{P}_{m}$ is $\left(d_{1}, \cdots, d_{m}\right)$.

More precisely, since $\tau_{\nu+1}>\tau_{\nu}$, the difference $\tau_{i+1}-\tau_{\nu}$, as a vector in $\mathbb{R}^{m}$, has the form $\left(q_{1}, \cdots, q_{m}\right)$ with $q_{i} \in \mathbb{Z}_{\geqslant 0}$, and we have a parallelotope of these dimensions with minimal vertex $\tau_{\nu}$ and maximal vertex $\tau_{\nu+1}$. See the right part of Fig. 8 for $m=2$.

It remains to notice that the poset of such block paths $\mathfrak{P}$ is identified with the poset of faces of $\amalg \mathrm{D}_{d_{1}, \cdots, d_{m}}$. For $m=2$ the reader can compare with the description of all faces of the shuffle polytope $N_{d_{1}, d_{2}}$ in [23] Ch. 12, $\S 2$ in terms of "labirinths": block paths are precisely the labirinths corresponding to faces of $N_{d_{1}, d_{2}}$ which are also faces of $P_{d_{1}+d_{2}}$.

This proves Propositions A.9 and A.8.

(A.4) Relation to XO-tables. Let $\left(d_{1}, \cdots, d_{m}\right)$ be as before.

Definition A.10. By an XO-table of type $\left(d_{1}, \cdots, d_{m}\right)$ we will mean an $l \times m$ matrix $\Xi$ for some $l \geqslant 1$ formed by X's and O's such that:

(1) The number of X's in the $j$ th column of $\Xi$ is precisely $d_{j}$.

(2) Each row of $\Xi$ contains at least one X.

The set of such tables will be denored $\mathrm{XO}\left(d_{1}, \cdots, d_{m}\right)$. 
The number $l=l(\Xi)$ will be called the height of $\Xi$. It can vary between $\max \left(d_{1}, \cdots, d_{m}\right)$ and $d_{1}+\cdots+d_{m}$.

Examples A.11. (a) XO-tables $\Xi$ of maximal height $d_{1}+\cdots+d_{m}$ are in bijection with $\amalg_{d_{1}, \cdots, d_{m}}$. In this case there is exactly one $\mathrm{X}$ in every row, so the columns give a shuffle.

(b) XO-tables of type $(1,2)$ are depicted in Fig. 9.

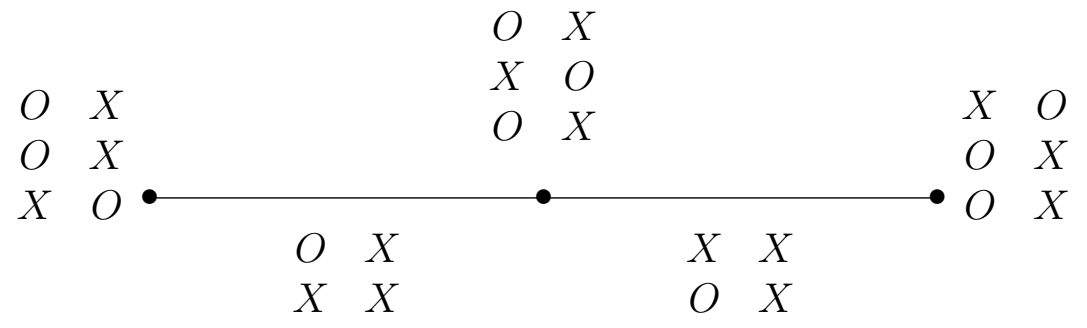

Figure 9: XO-tables of type $(1,2)$, depicted on vertices and edges to indicate contractions.

To an XO-table $\Xi$ we can associate an integer matrix $\widetilde{\Xi}$ of the same size by replacing each $\mathrm{X}$ with 1 and each $\mathrm{O}$ with 0 . For any $i=1, \cdots, l=l(\Xi)$ we denote by $\widetilde{\Xi}_{i, \bullet}$ the $i$ th row of $\Xi$, and similarly for $\Xi_{i \bullet}$.

Definition A.12. Let $\Xi, \Xi^{\prime}$ be two XO-table of the same type $\left(d_{1}, \cdots, d_{m}\right)$ of heights $l, l^{\prime}$ respectively. We say that $\Xi^{\prime}$ is a contraction of $\Xi$ and write $\Xi \leqslant \Xi^{\prime}$, if there following holds:

(C) There is a surjective monotone map $\varphi:\{1, \cdots, l\} \rightarrow\left\{1, \cdots, l^{\prime}\right\}$ such that for any $i^{\prime} \in\left\{1, \cdots, l^{\prime}\right\}$

$$
\widetilde{\Xi}_{i^{\prime}, \bullet}^{\prime}=\sum_{i \in \varphi^{-1}\left(i^{\prime}\right)} \widetilde{\Xi}_{i, \bullet}
$$

(summation of integer vectors).

We say that $\Xi$ is an elementary contraction of $\Xi$ if $\Xi \leqslant \Xi^{\prime}$ and $l^{\prime}=l-1$.

Since all the entries of $\widetilde{\Xi}, \widetilde{\Xi}^{\prime}$ are 0 's and 1's, the condition (C) means that the rows $\Xi_{i, \bullet}$, $i \in \varphi^{-1}\left(i^{\prime}\right)$, have mutually disjoint subsets of positions filled by X's, and the row $\Xi_{i^{\prime}, \bullet}^{\prime}$ is obtained by putting X's in all of these positions.

Proposition A.13. (a) $\leqslant$ is a partial order on $\mathrm{XO}_{d_{1}, \cdots, d_{m}}$ with minimal elements corresponding to shuffles (XO-tables of height $d_{1}+\cdots+d_{m}$ ).

(b) $\left(\mathrm{XO}_{d_{1}, \cdots, d_{m}}, \leqslant\right)$ is isomorphic to the poset of faces of $\amalg \mathrm{D}_{d_{1}, \cdots, d_{m}}$.

Proof: (a) is obvious. To prove (b), let us number the X's in an XO-table $\Xi$ from 1 to $d$ lexicographically, first from top to bottom in the first column, then similarly in the second column etc. Then each row will pick up a subset in $\{1, \cdots, d\}$. Denoting by $I_{\nu}$ the subset picked up by the $\nu$ th row, $\nu=1, \cdots, l=l(\Xi)$, we get a sequence $\left(I_{1}, \cdots, I_{l}\right)$ of subsets 
defining a face $\left[I_{1}, \cdots, I_{l}\right]$ of $P_{d}$ by Proposition A.1. All the vertices of $\left[I_{1}, \cdots, I_{l}\right]$ are $\left(d_{1}, \cdots, d_{m}\right)$-shuffles, so $\left[I_{1}, \cdots, I_{l}\right]$ is a face of $\amalg D_{d_{1}, \cdots, d_{m}}$. This establishes the claimed isomorphism of posets.

Definition A.14. The XO-cochain complex of type $\left(d_{1}, \cdots, d_{m}\right)$ is the cochain complex of $\mathbf{k}$-vector spaces defined as the graded space

$$
C^{\bullet}\left(\mathrm{XO}_{d_{1}, \cdots, d_{m}}\right)=\underset{\Xi \in \mathrm{XO}_{d_{1}, \cdots, d_{m}}}{\bigoplus} \mathbf{k}[l(\Xi)-d], \quad d=d_{1}+\cdots+d_{m}
$$

with the differential given by the alternating sum of elementary contractions.

Corollary A.15. The complex $C^{\bullet}\left(\mathrm{XO}_{d_{1}, \cdots, d_{m}}\right)$ is quasi-isomorphic to $\mathbf{k}$ in degree 0 .

Proof: Indeed, it is identified with the cellular cochain complex of $\amalg \mathrm{D}_{d_{1}, \cdots, d_{m}}$ which is contractible by Proposition A.8.

\section{References}

[1] D. Ayala, J. Francis, H. L. Tanaka. Factorization homology of stratified spaces. Selecta Math. (N.S.) 23 (2017) 293-362.

[2] B. Bakalov, A. Kirillov, Jr. Lectures on Tensor Categories and Modular Functors. Amer. Math. Soc. Publ. 2000.

[3] A. Bapat. Recollement for perverse sheaves on real hyperplane arrangements. arXiv:1810.13126.

[4] A. Beilinson. On the derived category of perverse sheaves. In: $K$-theory, arithmetic and geometry (Moscow, 1984), Lecture Notes in Math. 1289, Springer-Verlag, 1987, 27-41.

[5] A. Beilinson. How to glue perverse sheaves. In: $K$-theory, arithmetic and geometry (Moscow, 1984), Lecture Notes in Math. 1289, Springer-Verlag, 1987, 42 - 51.

[6] A. Beilinson, J.Bernstein, Localisation de g-modules, C. R. Acad. Sci Paris, 292 (1981), 15 $-18$.

[7] A. Beilinson, V. Drinfeld. Chiral Algebras. AMS Coll. Publ. 51, 2004.

[8] A.A. Beilinson, G. Lusztig, R. MacPherson. A geometric setting for the quantum deformation of $G L_{n}$. Duke Math. J. 61 (1990) 655-677.

[9] Y. N. Bespalov. Crossed modules and quantum groups in braided categories. Applied Categor. Structures 5 (1997) 155-204.

[10] R. Bezrukavnikov, M. Finkelberg, V. Schechtman. Factorizable Sheaves and Quantum Groups, Lecture Notes in Math. 1691, Springer-Verlag, 1998. 
[11] A. Björner, G. Ziegler. Combinatorial stratification of complex arrangements. Jour. AMS, 5 (1992), 105-149.

[12] K. Costello. Supersymmetric gauge theory and the Yangian. arXiv:1303.2632.

[13] K. Costello, O. Gwilliam. Factorization Algebras in Quantum Field Theory, vol. 1. Cambridge Univ. Press, 2017.

[14] P. Diaconis. A. Gangolli. Rectangular arrays with fixed margins, in: "Discrete Probability and Algorithms" (Minneapolis MN 1993) p. 15-41. IMA Vol. Math. Appl. 72, Springer-Verlag, 1995.

[15] J. S. Ellenberg, T. T. Tran, C. Westerland. Fox-Neuwirth-Fuks cells, quantum shuffle algebras and Malle's conjecture for functional fields. arXiv:1701.04541.

[16] J. S. Ellenberg, A. Venkatesh, C. Westerland. Homological stability for Hurwitz spaces and the Cohen-Lenstra heuristic over function fields. Ann. Math. 183 (2016) 729-786.

[17] B. L. Feigin, A.V. Odesskii. Vector bundles on elliptic curves and Sklyanin algebras, in: "Topics in quantum groups and finite-type invariants", p. 65-84, Amer. Math. Soc. Transl. Ser. 2, 185, Amer. Math. Soc., Providence, RI, 1998.

[18] R. Fox, L. Neuwirth. Braid groups. Math. Scand. 10 (1962) 119-126.

[19] D.B. Fuks. Cohomology of the braid group mod 2. Funkc. Anal. i Pril. 4 (1970), N. 2, 62-73.

[20] D. Gaitsgory. Notes on factorizable sheaves. <http://www.math.harvard.edu/ gaitsgde/GL/FS.pdf > (2008)

[21] D. Gaitgory, S. Lysenko. Metaplectic Whittaker category and quantum groups: the "small" FLE. arXiv:1903.02279.

[22] A. Galligo, M. Granger, Ph. Maisonobe. D-modules et faisceaux pervers dont le support singulier est un croisement normal. Ann. Inst. Fourier (Grenoble) 35 (1985), 1-48.

[23] I. M. Gelfand, M. M. Kapranov, A.V. Zelevinsky. Discriminants, Resultants and Multidimensional Determinants. Birhhäuser, Boston, 1994.

[24] I. M. Gelfand, Y.I. Manin. Methods of Homolocial Algebra. Springer-Verlag, Berlin, 2003.

[25] G. Ginot. Notes on factorization algebras, factorization homology and applications, in: "Mathematical aspects of quantum field theories" (D. Calaque, T. Strobl, Eds.) pp./ 429-552, Springer-Verlag, 2015. arXiv:1307.5213.

[26] M. Granger, Ph. Maisonobe. Faisceaux pervers relativement à un point de rebroussement. C.R. Acad. Sci. Paris. Sér. I 299 (1984), 567-570.

[27] A. Joyal, R. Street. Braided tensor categories. Adv. Math. 102 (1993), 20 - 78.

[28] T. Kadeishvili. On the cobar construction of a bialgebra. arXiv:math/0406502. 
[29] M. Kashiwara, P. Schapira. Sheaves on Manifolds. Springer-Verlag, 1990.

[30] M. Kashiwara, P. Schapira. Categories and Sheaves. Springer-Verlag, 2006

[31] M. Kashiwara, P. Schapira. Ind-sheaves. Astérisque 271 (2001).

[32] M. Kapranov, V. Schechtman. Perverse sheaves on real hyperplane arrangements. Ann. Math. 183 (2016), $619-679$.

[33] M. Kapranov, V. Schechtman. Perverse Schobers. Morfismos 22 (2018), 85 - 119; arXiv:1411.2772.

[34] M. Kapranov, V. Schechtman. Perverse sheaves and graphs on surfaces. arXiv:1601.07789.

[35] M. Kapranov, O. Schiffmann, E. Vasserot, The Hall algebra of a curve, Selecta Math. (N.S.) 23 (2017), 117-177.

[36] M. Kapranov, O. Schiffmann, E. Vasserot, Spherical Hall algebra of $\overline{\operatorname{Spec}(\mathbb{Z})}$, in: "Homological mirror symmetry and tropical geometry" p. 153-196, Lect. Notes Unione Mat. Ital. 15, Springer-Verlag, 2014.

[37] M. Kapranov, V. Voevodsky. 2-categories and Zamolodchikov tetrahedra equations, Proc. Symp. Pure Math. 56, Part 2 (1994), 177 - 259.

[38] M. Kontsevich, Y. Soibelman. Cohomological Hall algebra, exponential Hodge structures and motivic Donaldson-Thomas invariants. Comm. Numb. Theory and Physics 5 (2011), Number 2, 231-352 (arXiv:1006.2706).

[39] J. Lambek. Lectures on Rings and Modules. Amer. Math. Soc. 2009.

[40] J.-L.Loday, M.Ronco, On the structure of cofree Hopf algebras, arXiv:math/0405330.

[41] J. Lurie. Higher Algebra. <www.math.harvard.edu/ lurie/papers/HA.pdf>

[42] G. Lusztig. Quantum Groups. Birkhäuser, Boston, 1993.

[43] G. Lusztig. Quantum Groups at roots of 1. Geom. Dedicata 35 (1990) 89-114.

[44] G. Lusztig. Exotic Fourier transform. Duke Math. J. 73 (1994), 227 - 241.

[45] J. McClure, J. Smith. A solution of Deligne's Hochschild cohomology conjecture. Contemp. Math. 293 (2002) 153-193, arXiv:math/9910126.

[46] R. MacPherson, K. Vilonen. Perverse sheaves with singularities along the curve $y^{n}=x^{m}$. Comment. Math. Helv. 63 (1988), 89-102.

[47] S. Majid. Algebras and Hopf algebras in braided categories, in: "Advances in Hopf algebras", Marcel Dekker Lect. Notes in Pure and Appl. Math. 158(1994) 55-105, arXiv q-alg/9509023.

[48] J. P.May. Geometry of iterated loop spaces. Lecture Notes in Math. 271, Springer-Verlag, 1972. 
[49] W.D. Nichols. Bialgebras of type one. Comm. Algebra 6 (1978), 1521-1552.

[50] T. K. Petersen. A two-sided analog of the Coxeter complex. arXiv:1607.00086.

[51] M. Rosso. Certaines formes bilinéaires sur les groupes quantiques et une conjecture de Schechtman et Varchenko, C. R. Acad. Sci. Paris 314 (1992), 5 - 8.

[52] M. Rosso. Quantum groups and quamtum shuffles. Invent. Math. 133 (1998) 399-416.

[53] V. Schechtman, A. Varchenko, Quantum groups and homology of local systems, Algebraic geometry and Analytic geometry, ICM-90 Satellite conference proceedings, pp. 182 - 197.

[54] Y. Soibelman. Meromorphic tensor categories. arXiv:q-alg/9709030.

[55] M. Takeuchi. Finite Hopf algebras in braided tensor categories. J. Pure Appl. Alg.138 (1999) 59-82.

[56] K.Takeuchi, Dimension formula for hyperfunction solutions to holonomic $\mathcal{D}$-modules. Adv. Math. 180 (2003), 134-145.

[57] D. Tamarkin. Another proof of M. Kontsevich formality theorem for $\mathbb{R}^{n}$. arXiv:math/9803025,

[58] D. Tamarkin, Quantization of Lie bialgebras via the formality of the operad of little discs, GAFA 17 (2007), 537 - 604.

[59] D. Treumann. Exit paths and constructible stacks. Compositio Math. 145 (2009) 1504-1532.

[60] M. H. Weissman. Equivariant perverse sheaves on Coxeter arrangements and buildings. arXiv:1706.07847.

[61] D. Yetter. Quantum groups and representations of monoidal categories. Proc. Cambridge Phil. Soc. 108 (1990) 261-290.

M.K.: Kavli IPMU, 5-1-5 Kashiwanoha, Kashiwa, Chiba, 277-8583 Japan, mikhail . kapranov@ipmu .jp

V.S.: Institut de Mathématiques de Toulouse, Université Paul Sabatier, 118 route de Narbonne, 31062 Toulouse, France, schechtman@math.ups-tlse.fr 